\title{
Lectures on the Orbital Stability of Standing Waves and Application to the Nonlinear Schrödinger Equation
}

\author{
C.A. Stuart
}

\begin{abstract}
In the first part of these notes, we deal with first order Hamiltonian systems in the form $J u^{\prime}(t)=\nabla H(u(t))$ where the phase space $X$ may be infinite dimensional so as to accommodate some partial differential equations. The Hamiltonian $H \in C^{1}(X, \mathbb{R})$ is required to be invariant with respect to the action of a group $\left\{e^{t A}: t \in \mathbb{R}\right\}$ of isometries where $A \in B(X, X)$ is skew-symmetric and $J A=A J$. A standing wave is a solution having the form $u(t)=e^{t \lambda A} \varphi$ for some $\lambda \in \mathbb{R}$ and $\varphi \in X$ such that $\lambda J A \varphi=\nabla H(\varphi)$. Given a solution of this type, it is natural to investigate its stability with respect to perturbations of the initial condition. In this context, the appropriate notion of stability is orbital stability in the usual sense for a dynamical system. We present some of the important criteria for establishing orbital stability of standing waves.

In the second part we consider the nonlinear Schrödinger equation which provides an interesting example of this situation where standing waves appear as time-harmonic solutions. We show how the general theory applies to this case and review what is known about stability.
\end{abstract}

Mathematics Subject Classification (2000). Primary 37-01; Secondary 37K45, 35Q55.

Keywords. Orbital stability, Hamiltonian system, nonlinear Schrödinger equation. 


\section{Contents}

1. Introduction 331

2. Setting and problem 335

2.1. Variational triple $\quad 335$

2.2. Hamiltonian system 336

2.3. Invariance of the Hamiltonian 337

2.4. Standing waves 339

2.5. Orbits and orbital stability 340

2.6. Well-posedness of IVP 342

2.7. Summary 345

3. Sufficient conditions for the orbital stability of a standing wave 349

4. Proof of the main stability theorem 352

4.1. Change of variable 353

4.2. $\Gamma$-invariant Lyapunov functions 355

4.3. Existence of a $\Gamma$-invariant Lyapunov function 357

4.4. Stability of the stationary solution $u \equiv 0 \quad 359$

5. A branch of standing waves 360

5.1. The Vahkitov-Kolokolov criterion 363

6. Constrained minimization and stability 368

6.1. A weaker notion of stability 370

7. The nonlinear Schrödinger equation 372

7.1. Expressing (CNLS) in the form (2.1) 374

7.2. Solutions of the stationary equation 377

7.3. The stability conditions 378

7.4. Summary 381

8. NLS with a power-law nonlinearity 383

8.1. Bifurcation from an eigenvalue 384

8.2. Global branch in the defocusing case 391

8.3. Global branch in the self-focusing case 392

8.4. The case where $P$ is constant 394

8.5. Conditions ensuring weak stability 395

References $\quad 397$

Acknowledgment 399 


\section{Introduction}

These notes are concerned with the orbital stability of certain special solutions of a first order Hamiltonian system acting on a real Hilbert space, $X$. The Hamiltonian is required to be invariant under the action of a group $\{T(\theta): \theta \in \mathbb{R}\}$ of isometries on $X$ that preserves the symplectic form, in the sense that $\omega(T(\theta) \xi, T(\theta) \eta)=\omega(\xi, \eta)$ for all $\xi, \eta \in X$ and $\theta \in \mathbb{R}$. In other words, the isometry $T(\theta): X \rightarrow X$ is also a symplectic map. We consider special solutions of the form $u(t)=T(\lambda t) \xi$ for some $\lambda \in \mathbb{R}$ and $\xi \in X$ and their stability with respect to perturbation of the initial condition $u(0)=\xi$.

To present rapidly this underlying structure, let $X=\mathbb{R}^{2 N}$ with the usual scalar product $\langle\cdot, \cdot\rangle$ and consider the system

$$
J u^{\prime}(t)=\nabla H(u(t))
$$

where $J=-J^{T}=-J^{-1}$ is a real $2 N \times 2 N$ matrix and $H \in C^{2}(X, \mathbb{R})$. Then $\frac{d}{d t} H(u(t))=\left\langle\nabla H(u(t)), u^{\prime}(t)\right\rangle=\left\langle J u^{\prime}(t), u^{\prime}(t)\right\rangle=0$, showing that $H(u(t))$ is independent of $t$ whenever $u$ is a solution of (1.1). Let $\Gamma=\{T(\theta): \theta \in \mathbb{R}\}$ be a group of isometries on $\mathbb{R}^{2 N}$ with infinitesimal generator $A=T^{\prime}(0)$ so that $T(\theta)=e^{\theta A}$ and $T^{\prime}(\theta)=A T(\theta)$ for all $\theta$. Then $A^{T}=-A$ and $T(\theta)^{T}=T(\theta)^{-1}=T(-\theta)$. The invariance of $H$ with respect to $\Gamma$ becomes

$$
\begin{aligned}
& H(T(\theta) \xi)=H(\xi) \text { for all } \theta \in \mathbb{R} \text { and } \xi \in \mathbb{R}^{2 N} \\
\Leftrightarrow & \langle A \xi, \nabla H(\xi)\rangle=0 \text { for all } \xi \in \mathbb{R}^{2 N} .
\end{aligned}
$$

For the symplectic form $\omega$ defined by $\omega(\xi, \eta)=\langle J \xi, \eta\rangle$, we have that

$$
\begin{aligned}
& \omega(T(\theta) \xi, T(\theta) \eta)=\omega(\xi, \eta) \text { for all } \xi, \eta \in X \text { and } \theta \in \mathbb{R} \\
\Leftrightarrow & T(\theta)^{T} J T(\theta)=J, \quad(\text { i.e. } T(\theta) \text { is symplectic) for all } \theta \in \mathbb{R} \\
\Leftrightarrow & J T(\theta)=T(\theta) J \text { for all } \theta \in \mathbb{R}, \text { since } T(\theta)^{T}=T(\theta)^{-1} \\
\Leftrightarrow & J A=A J .
\end{aligned}
$$

The $\Gamma$-invariance of $H$ implies that $\nabla H$ is $\Gamma$-equivariant:

$$
\nabla H(T(\theta) \xi)=T(\theta) \nabla H(\xi) \text { for all } \theta \in \mathbb{R} \text { and } \xi \in \mathbb{R}^{2 N} .
$$

For any fixed $\lambda \in \mathbb{R}$, we set $u(t)=T(\lambda t) v(t)$ and, from the equivariance of $\nabla H$, it follows that $u$ satisfies (1.1) if and only if $v$ satisfies

$$
J v^{\prime}(t)=\nabla H(v(t))-\lambda J A v(t) .
$$


Since $(J A)^{T}=A^{T} J^{T}=A J=J A$, we see that (1.2) is a Hamiltonian system with Hamiltonian

$$
G_{\lambda}(\xi)=H(\xi)-\lambda Q(\xi) \text { where } Q(\xi)=\frac{1}{2}\langle J A \xi, \xi\rangle=\frac{1}{2} \omega(A \xi, \xi) .
$$

If $u(t)$ is a solution of $(1.1), H(u(t))$ and $G_{\lambda}(v(t))$ are both independent of $t$. But, by the invariance of $H$,

$$
G_{\lambda}(v(t))=H(T(-\lambda t) u(t))-\lambda Q(v(t))=H(u(t))-\lambda Q(v(t)),
$$

from which we see that $\lambda Q(v(t))$ is also independent of $t$. With this in mind, we set $B=J A(=A J)$ so that $Q(\xi)=\frac{1}{2}\langle B \xi, \xi\rangle$. Then $B^{T}=B$ and, for any solution of (1.1),

$$
\frac{d}{d t} Q(u)=\left\langle B u, u^{\prime}\right\rangle=\left\langle J A u, J^{-1} \nabla H(u)\right\rangle=-\langle A u, \nabla H(u)\rangle=0,
$$

whereas, for any $\xi \in \mathbb{R}^{2 N}$,

$$
\frac{d}{d \theta} Q(T(\theta) \xi)=\left\langle B T(\theta) \xi, T^{\prime}(\theta) \xi\right\rangle=\langle J A T(\theta) \xi, A T(\theta) \xi\rangle=0,
$$

showing that, like $H, Q$ is also a $\Gamma$-invariant conserved quantity for (1.1). In fact, $Q$ is also $J$-invariant since, for all $\xi \in \mathbb{R}^{2 N}$,

$$
Q(J \xi)=\frac{1}{2}\langle B J \xi, J \xi\rangle=\frac{1}{2}\langle J A J \xi, J \xi\rangle=\frac{1}{2}\langle B \xi, \xi\rangle=Q(\xi) .
$$

We have shown that, for any $\lambda \in \mathbb{R}$, the (canonical) change of variable $u(t)=T(\lambda t) v(t)$ transforms (1.1) into (1.2). If $v(t) \equiv \xi$ is a stationary solution of (1.2) for some $\lambda \in \mathbb{R}$, we obtain a special solution of the Hamiltonian system (1.1) having the form

$$
u(t)=T(\lambda t) \xi \text { for some } \lambda \in \mathbb{R} \text { and } \xi \in X .
$$

Solutions of this kind will be referred to as standing waves since this is the terminology that is commonly adopted in the context of the nonlinear Schrödinger equation. For $X=\mathbb{R}^{2 N}$, they are either periodic or quasiperiodic functions of $t$, but there may well be periodic or quasi-periodic solutions that are not of the form (1.3). One finds that $u(t)=T(\lambda t) \xi$ satisfies the Hamiltonian system (1.1) if and only of

$$
\nabla H(\xi)=\lambda B \xi
$$

and solutions $\xi \in X$ of the "stationary equation" (1.4) occur as critical points of the functional $G_{\lambda}(u)=H(u)-\lambda Q(u)$. We concentrate on standing waves that are not stationary solutions of (1.1) and this requires $\lambda A \xi \neq 0$. 
In this way two fundamental problems appear. Firstly to establish the existence of standing waves and secondly to investigate their stability as solutions of (1.1). Since (1.1) is autonomous and a standing wave is periodic or quasi-periodic, the only type of stability that can be hoped for is orbital stability. These lectures present some results concerning these issues.

In Section 2 we begin by posing the problem in a setting where $X$ can be an infinite dimensional real Hilbert space. In its most general form, this would involve dealing with unbounded operators. By restricting to a situation where $X$ is part of a variational triple, we gain some simplifications while retaining sufficient generality to deal with problems from partial differential equations.

Some basic results concerning the orbital stability of standing waves are formulated in Section 3 and the proof of the main theorem is given in Section 4 by the construction of a Lyapunov function. In Section 5 we deal with a frequently occurring situation where the standing wave in question is embedded in a one-parameter family of standing waves. This covers the so-called Vakhitov-Kolokolov condition and the fundamental contributions by M. Weinstein and Grillakis-Shatah-Strauss. We complete the discussion of the abstract problem in Section 6 by reviewing a method of constrained minimization for finding standing waves and a weaker notion of stability which accompanies this approach.

In Section 7 we introduce the nonlinear Schrödinger equation and show how it fits into the general structure presented earlier. Finally, in Section 8 , we review what can be deduced about the existence and orbital stability of standing waves in the case of a standard power-law nonlinearity. Translational invariance of the nonlinear Schrödinger equation with respect to the spatial variables has to be excluded in order to obtain orbital stability of standing waves in the strict sense of dynamical systems. There is a rich literature dealing with weaker notions of orbital stability which can be satisfied in cases where the Hamiltonian in invariant with respect to additional symmetries, see [15]. We do not explore this topic, but as is shown in Section 8.5, the weaker notion of stabilty introduced in Section 6 does give some information about spatially invariant Schrödinger equations.

We have refrained from any discussion of conditions implying instability, but of course this forms an essential complement to the results collected below. For this aspect of the theory we simply refer to [4], [6], [12], [16], [17], [12], [25], [36] and the references therein. Similarly, we do not attempt 
to describe work on more detailed analysis of the asymptotic behaviour of solutions near standing waves. See [28], [33] for a review of this.

At the beginning of this introduction we required the Hamiltonian $H$ to be invariant under the action of a group $\Gamma$ of isometries that preserves the symplectic structure and we showed that, when $X=\mathbb{R}^{2 N}$, this implies the existence of a $\Gamma$-invariant quadratic conserved quantity $Q$ which is also $J$-invariant. In fact, conversely, the existence of a quadratic function $Q$, which is conserved by (1.1) and is $J$-invariant, implies the invariance of $H$ under the action of a group of isometries which is determined by $Q$. To see this, consider (1.1) with $X=\mathbb{R}^{2 N}$ and suppose that there is a second conserved quantity $Q$ that is quadratic in $\xi$ and $J$-invariant:

$$
Q(\xi)=\frac{1}{2}\langle B \xi, \xi\rangle \text { and } Q(J \xi)=Q(\xi) \text { for all } \xi \in X
$$

where, without loss of generality, we may suppose $B$ is a real $2 N \times 2 N$ symmetric matrix. Noting that

$$
Q(J \xi)=Q(\xi) \text { for all } \xi \in X \Longleftrightarrow J B=B J,
$$

we define a real $2 N \times 2 N$ skew-symmetric matrix by $A=-J B$ and, since $Q$ is conserved,

$$
\begin{aligned}
0 & =\frac{d}{d t} Q(u(t))=\left\langle B u(t), u^{\prime}(t)\right\rangle \\
& =-\langle B u(t), J \nabla H(u(t))\rangle=-\langle A u(t), \nabla H(u(t))\rangle,
\end{aligned}
$$

for all solutions of (1.1). Consequently,

$$
\langle\nabla H(\xi), A \xi\rangle=0 \text { for all } \xi \in X .
$$

Since $A$ is skew-symmetric, $T(\theta) \equiv e^{\theta A}: X \rightarrow X$ is an isometric isomorphism for all $\theta \in \mathbb{R}$ and

$$
\begin{aligned}
\frac{d}{d \theta} H(T(\theta) \xi) & =\left\langle\nabla H(T(\theta) \xi), T^{\prime}(\theta) \xi\right\rangle=\langle\nabla H(T(\theta) \xi), A T(\theta) \xi\rangle \\
& =0 \text { for all } \xi \in X,
\end{aligned}
$$

showing that $H$ is $\Gamma$-invariant, where $\Gamma=\{T(\theta): \theta \in \mathbb{R}\}$. Furthermore, the $J$-invariance of $Q$ implies that $J B=B J$, from which it follows that $J A=A J$ and hence that $\Gamma$ preserves the symplectic structure induced by $J$. 


\section{Setting and problem}

We begin by recalling the notion of variational triple that is often used in dealing with partial differential equations. Then we define a Hamiltonian system in a context which is broad enough to include some partial differential equation such as the nonlinear Schrödinger equation. Because of issues concerning the regularity of solutions, it is not appropriate to simply use (1.1) when $\operatorname{dim} X=\infty$. We consider Hamiltonian systems which are invariant under a group action on $X$, extending to our context what was sketched in the introduction when $\operatorname{dim} X<\infty$. Standing waves are special solutions of this equation and they satisfy a generalization of (1.4). We then review the notion of orbit and orbital stability. This necessitates a discussion of the well-posedness of the initial value problem for Hamiltonian systems in our setting.

We end this section by summarizing the basic hypotheses which will be assumed to hold throughout the rest of the presentation without further comment.

\subsection{Variational triple}

Let $(H,(\cdot, \cdot),\|\cdot\|)$ and $\left(X,\langle\cdot, \cdot\rangle_{X},\|\cdot\|_{X}\right)$ be real Hilbert spaces with dual spaces $H^{*}$ and $X^{*}$. They form a variational triple when, identifying $H$ with $H^{*}$ via their Riesz isomorphism, we have

$$
X \subset H=H^{*} \subset X^{*}
$$

where the inclusions are dense and the embeddings are continuous. The duality between $X^{*}$ and $X$ will be denoted by

$$
\langle f, u\rangle=f(u) \text { for all } f \in X^{*} \text { and } u \in X .
$$

The Riesz (isometric) isomorphism between $X$ and $X^{*}$ will be denoted by $R: X \rightarrow X^{*}$,

$$
\langle R u, v\rangle=\langle u, v\rangle_{X} \text { for all } u, v \in X .
$$

Then $\left(X^{*},\langle\cdot, \cdot\rangle_{*},\|\cdot\|_{*}\right)$ is a Hilbert space with $\langle f, g\rangle_{*}=\left\langle R^{-1} f, R^{-1} g\right\rangle_{X}$ for all $f, g \in X^{*}$. For $L \in B(X, X)$, its dual operator is $L^{*}=R L^{T} R^{-1} \in$ $B\left(X^{*}, X^{*}\right)$ where $L^{T}$ is the adjoint of $L$ in $X$. Thus

$$
\langle L u, v\rangle_{X}=\left\langle u, L^{T} v\right\rangle_{X} \text { and }\left\langle L^{*} f, v\right\rangle=\langle f, L v\rangle \text { for all } u, v \in X \text { and } f \in X^{*} \text {. }
$$

Since

$$
|(u, v)| \leq\|u\|\|v\| \leq C\|u\|_{X}\|v\|_{X} \text { for all } u, v \in X,
$$


there is a natural injection $\mathfrak{I} \in B\left(X, X^{*}\right)$ such that

$$
\langle\Im u, v\rangle=(u, v) \text { for all } u, v \in X .
$$

The two scalar products on $X$ are related by

$$
(u, v)=\langle\Im u, v\rangle=\left\langle R^{-1} \Im u, v\right\rangle_{X} \text { for all } u, v \in X .
$$

Setting $S=R^{-1} \mathfrak{I}$, we find that $S \in B(X, X), S^{T}=S$ and $\langle S u, u\rangle_{X}>0$ for all $u \in X \backslash\{0\}$.

Examples (1) If $\left(X,\langle\cdot, \cdot\rangle_{X}\right)$ is a real, finite dimensional Hilbert space, we obtain a variational triple with $H=X$ and $R=\mathfrak{I}=S=I$.

(2) In the usual notation for Sobolev spaces, $H^{1}\left(\mathbb{R}^{N}\right) \subset L^{2}\left(\mathbb{R}^{N}\right) \subset$ $H^{-1}\left(\mathbb{R}^{N}\right)$ is a variational triple where $R=-\Delta+1$ and $S=(-\Delta+1)^{-1}$. This will be used later to treat the nonlinear Schrödinger equation.

\subsection{Hamiltonian system}

We define a Hamiltonian system on the real Hilbert space $\left(X,\langle\cdot, \cdot\rangle_{X},\|\cdot\|_{X}\right)$ in the following way. Given $J$ and $H$ satisfying

(H1) $J \in B(X, X)$ with $J^{T}=J^{-1}=-J$ and $H \in C^{2}(X, \mathbb{R})$,

we consider the initial value problem

$$
\begin{aligned}
\frac{d}{d t}(\Im u) & =J^{*} H^{\prime}(u) \text { in } X^{*}, \\
u(0) & =u_{0}
\end{aligned}
$$

where $H^{\prime}(\xi) \in X^{*}$ denotes the Fréchet derivative of $H$ at $\xi \in X$ and $u_{0} \in X$. A solution of (IVP) is a function defined on an interval $[0, b)$ for some $b>0$ such that

$$
u \in C([0, b), X) \text { and } \Im u \in C^{1}\left((0, b), X^{*}\right)
$$

with $u(0)=u_{0} \in X$ and

$$
\frac{d}{d t}(\Im u(t))=J^{*} H^{\prime}(u(t))
$$

for all $t \in(0, b)$. We shall return to the discussion of well-posedness of (IVP) once we have introduced the invariance of $H$ with respect to a group action.

Noting that $J^{*}=R J^{T} R^{-1}$, we have that $\left[J^{*}\right]^{-1}=R\left[J^{T}\right]^{-1} R^{-1}=$ $R J R^{-1}=-J^{*}$, so the differential equation can also be written as $R J R^{-1} \frac{d}{d t}(\Im u(t))=H^{\prime}(u(t))$. Using $\nabla H$ to denote the gradient of $H$ in the sense that

$$
\left\langle H^{\prime}(v), w\right\rangle=\langle\nabla H(v), w\rangle_{X} \text { for all } v, w \in X,
$$


we have that $H^{\prime}(v)=R \nabla H(v)$ and the equation becomes

$$
J R^{-1} \frac{d}{d t} \Im u(t)=\nabla H(u(t)) \text { in } X .
$$

This reduces to $(1.1)$ when $(H,(\cdot, \cdot),\|\cdot\|)=\left(X,\langle\cdot, \cdot\rangle_{X},\|\cdot\|_{X}\right)$ since then $R=\mathfrak{I}=I$.

Remark. We must acknowledge that our assumption that $J \in B(X, X)$ restricts the range of applications that we can cover. For example [14], the Korteweg-de Vries equation can be expressed as a Hamiltonian system with $J=\partial_{x}$, but then $J$ is unbounded. In this way the pioneering work of Benjamin and Bona concerning the stability of solitary waves for the KdV equation can be rederived from a generalization of the results presented below, [2], [3], [23], [36].

\subsection{Invariance of the Hamiltonian}

We introduce the invariance of $H$ through an operator $A$ having the following properties.

(H2) $A \in B(X, X)$ with $A^{T}=-A$ is such that

$$
A J=J A \text { and }\left\langle H^{\prime}(v), A v\right\rangle=0 \text { for all } v \in X .
$$

The skew-symmetric operator $A$ generates a group of isometries, $\Gamma=\{T(\theta): \theta \in \mathbb{R}\}$, where

$$
T: \mathbb{R} \rightarrow B(X, X) \text { is defined by } T(\theta)=e^{\theta A} .
$$

Then,

$$
T^{\prime}(\theta)=A T(\theta)=T(\theta) A, T(\theta)^{-1}=T(\theta)^{T}=T(-\theta) \text { and } T(\theta) J=J T(\theta)
$$

for all $\theta \in \mathbb{R}$.

For any function $F \in C^{1}(X, \mathbb{R})$, we have that

$$
\begin{aligned}
& \frac{d}{d \theta} F(T(\theta) v)=\left\langle F^{\prime}(T(\theta) v), T^{\prime}(\theta) v\right\rangle=\left\langle F^{\prime}(T(\theta) v), A T(\theta) v\right\rangle \\
& \quad \text { for all } \theta \in \mathbb{R} \text { and } v \in X .
\end{aligned}
$$

Hence $F(T(\theta) v)=F(T(0) v)=F(v)$ for all $\theta \in \mathbb{R}$ and $v \in X$ if and only if $\left\langle F^{\prime}(w), A w\right\rangle=0$ for all $w \in X$. In this case, $F$ is $\Gamma$-invariant and we have that

$$
\left\langle F^{\prime}(v), w\right\rangle=\left\langle F^{\prime}(T(\theta) v), T(\theta) w\right\rangle=\left\langle T(\theta)^{*} F^{\prime}(T(\theta) v), w\right\rangle \text { for all } v, w \in X,
$$

so $F^{\prime}$ is $\Gamma$-equivariant in the sense that

$$
T(-\theta)^{*} F^{\prime}(v)=F^{\prime}(T(\theta) v) \text { since }\left[T(\theta)^{*}\right]^{-1}=T(-\theta)^{*} \text { for all } \theta \in \mathbb{R} .
$$


The hypothesis (H2) ensures that $\|\cdot\|_{X}^{2}$ and $H$ are $\Gamma$-invariant since

$$
\langle w, A w\rangle_{X}=0 \text { and }\left\langle H^{\prime}(w), A w\right\rangle=0 \text { for all } w \in X .
$$

A symplectic form is defined on $X$ by setting

$$
\omega_{X}(\xi, \eta)=\langle J \xi, \eta\rangle_{X} \text { for } \xi, \eta \in X .
$$

Then, for all $\theta \in \mathbb{R}$,

$$
\omega_{X}(T(\theta) \xi, T(\theta) \eta)=\langle J T(\theta) \xi, T(\theta) \eta\rangle_{X}=\langle T(\theta) J \xi, T(\theta) \eta\rangle_{X}=\langle J \xi, \eta\rangle_{X}
$$

showing that $\omega_{X}$ is preserved by $\Gamma$.

Thus (H1) and (H2) ensure that $H$ is invariant with respect to a group $\Gamma$ of isometries on $X$ and that $\Gamma$ preserves the symplectic form associated with $J$.

In order to ensure that $\|\cdot\|^{2}$ is also a $\Gamma$-invariant quantity, we suppose that

(H3) $(A v, w)=-(v, A w)$ for all $v, w \in X$.

Then

$$
\frac{d}{d \theta}\|T(\theta) v\|^{2}=2\left(T(\theta) v, T^{\prime}(\theta) v\right)=2(T(\theta) v, A T(\theta) v)=0
$$

for all $\theta \in \mathbb{R}$ and $v \in X$ as required. It then follows that

$$
(T(\theta) v, w)=(v, T(-\theta) w) \text { for all } \theta \in \mathbb{R} \text { and } v, w \in X
$$

and so $\Gamma$ acts isometrically on $X$ with respect to $\|\cdot\|$, as well as $\|\cdot\|_{X}$. Recalling that $S=R^{-1} \mathfrak{I}$, it is easy to check that (H3) is equivalent to $A^{T} S=-S A$ and hence, when $A=-A^{T}$, to $A S=S A$.

Remark. Clearly, (H2) and (H3) are always satisfied by $A=0$, but in this case $T(\theta)=I$ for all $\theta \in \mathbb{R}$ and the associated standing waves are stationary solutions. We are interested Hamiltonians $H$ for which there exists $A \neq 0$ satisfying (H2) and (H3). That is, $H$ is invariant under the action of a nontrivial group of isometries $\{T(\theta): \theta \in \mathbb{R}\}$, which preserves the symplectic structure associated with $J$, and $A$ is its infinitesimal generator. 


\subsection{Standing waves}

Under the assumptions (H1) and (H2), a standing wave is a solution of (2.1) having the form

$$
u(t)=T(\lambda t) \xi \text { for all } t \in \mathbb{R} \text {, for some } \lambda \in \mathbb{R} \text { and } \xi \in X .
$$

In this case, $\xi$ is the initial value. For $u(t)=T(\lambda t) \xi$ we have that $u \in$ $C^{1}(\mathbb{R}, X)$ and so $\Im u \in C^{1}\left(\mathbb{R}, X^{*}\right)$ with

$$
\frac{d}{d t} \mathfrak{I} u(t)=\mathfrak{I} \frac{d}{d t} u(t)=\mathfrak{I} T^{\prime}(\lambda t) \lambda \xi=\lambda \mathfrak{I} T(\lambda t) A \xi .
$$

Using (H3), we find that, for all $v \in X$,

$$
\begin{aligned}
\left\langle\frac{d}{d t} \Im u(t), v\right\rangle & =\lambda(T(\lambda t) A \xi, v)=-\lambda(\xi, A T(-\lambda t) v) \\
& =-\lambda\left\langle T(-\lambda t)^{*} A^{*} \Im \xi, v\right\rangle
\end{aligned}
$$

so that $\frac{d}{d t} \Im u(t)=-\lambda T(-\lambda t)^{*} A^{*} \Im \xi$. On the other hand, by the $\Gamma$-equivariance of $H^{\prime}$,

$$
J^{*} H^{\prime}(u(t))=J^{*} H^{\prime}(T(\lambda t) \xi)=J^{*} T(-\lambda t)^{*} H^{\prime}(\xi)=T(-\lambda t)^{*} J^{*} H^{\prime}(\xi),
$$

showing that, for $u(t)=T(\lambda t) \xi$,

$$
(2.1) \Longleftrightarrow-\lambda A^{*} \Im \xi=J^{*} H^{\prime}(\xi) \Leftrightarrow \lambda J^{*} A^{*} \Im \xi=H^{\prime}(\xi) .
$$

Setting

$$
B=J^{*} A^{*} \mathfrak{I} \in B\left(X, X^{*}\right),
$$

we have that $u(t)=T(\lambda t) \xi$ is a solution of (2.1) if and only if

$$
H^{\prime}(\xi)-\lambda B(\xi)=0 .
$$

Now

$$
\langle B v, w\rangle=\left\langle J^{*} A^{*} \Im v, w\right\rangle=\langle\mathfrak{I} v, A J w\rangle=(v, A J w),
$$

so to maintain the variational structure of the problem we suppose that

(H4) $(J v, w)=-(v, J w)$ for all $v, w \in X$.

Then $(v, A J w)=(J A v, w)=(A J v, w)=\langle B w, v\rangle$. Thus $B \in B\left(X, X^{*}\right)$ is now symmetric in the sense that

$$
\langle B v, w\rangle=\langle B w, v\rangle \text { for all } v, w \in X,
$$


and we also have that

$$
\begin{aligned}
\langle B v, w\rangle & =(v, A J w)=(J A v, w)=\langle\mathfrak{I} J A v, w\rangle \text { so that } \\
B & =J^{*} A^{*} \mathfrak{I}=\mathfrak{I} J A .
\end{aligned}
$$

Defining $Q: X \rightarrow \mathbb{R}$ by

$$
Q(v)=\frac{1}{2}\langle B v, v\rangle \text { for all } v \in X,
$$

we have that $Q^{\prime}=B$.

Hence $u(t)=T(\lambda t) \xi$ is a standing wave if and only if $(\lambda, \xi)$ satisfies the stationary equation (2.3) which can be expressed as

$$
H^{\prime}(\xi)-\lambda Q^{\prime}(\xi)=0 .
$$

Defining an augmented Hamiltonian $G_{\lambda}: X \rightarrow \mathbb{R}$ by

$$
G_{\lambda}(v)=H(v)-\lambda Q(v)
$$

we see that, under the assumptions (H1) to (H4), standing waves are generated by critical points of $G_{\lambda}$.

Noting that

$$
\left\langle Q^{\prime}(v), A v\right\rangle=\left\langle J^{*} A^{*} \Im v, A v\right\rangle=\langle\Im J J v, A v\rangle=(J A v, A v)=0,
$$

it follows that $Q$ and hence $G_{\lambda}$ are $\Gamma$-invariant. Thus, if $\xi \in X$ is a critical point of $G_{\lambda}$, then so is $T(\theta) \xi$ for all $\theta \in \mathbb{R}$.

\subsection{Orbits and orbital stability}

For any $v \in X$,

$$
\Theta(v)=\{T(\theta) v: \theta \in \mathbb{R}\}
$$

is the orbit of $v$ under the action of the group $\Gamma=\{T(\theta): \theta \in \mathbb{R}\}$. Of course, $\Theta(v)$ is a bounded subset of $X, v \in \Theta(v)$ and

$$
\Theta(v)=\{v\} \Leftrightarrow A v=0 .
$$

When $T: \mathbb{R} \rightarrow B(X, X)$ is periodic, $\Theta(v)$ is a compact subset of $X$, otherwise $T$ is injective and $\Theta(v)$ may not be compact. When $T$ is quasi-periodic, $\Theta(v)$ may not even be a closed subset of $X$.

If $\xi \in X$ is a critical point of $G_{\lambda}$, then $\Theta(\xi)$ is also the orbit of the standing wave $u(t)=T(\lambda t) \xi$ in the sense of dynamical systems. This standing wave is orbitally stable if, for all $\varepsilon>0$, there exists $\delta>0$ such that for all initial conditions $u_{0} \in X$ with $\left\|\xi-u_{0}\right\|_{X}<\delta$, (IVP) has a unique 
maximal solution $u(t)$, it is defined for all $t \geq 0$ and $d(u(t), \Theta(\xi))<\varepsilon$ for all $t \geq 0$ where

$$
d(v, \Theta(\xi))=\inf \left\{\|v-T(\theta) \xi\|_{X}: \theta \in \mathbb{R}\right\} .
$$

Note that the standing wave is a stationary solution if and only if $\lambda A \xi=0$.

For any $\xi \in X$ and $\rho>0$, let

$$
\Theta(\xi)_{\rho}=\{v \in X: d(v, \Theta(\xi))<\rho\} .
$$

That is $\Theta(\xi)_{\rho}=\cup_{\theta \in \mathbb{R}} B(T(\theta) \xi, \rho)$ where $B(w, \rho)=\left\{v \in X:\|v-w\|_{X}<\right.$ $\rho\}$.

Since

$$
\|\eta-T(\theta) \xi\|_{X}=\|T(-\theta) \eta-\xi\|_{X} \text { for all } \theta \in \mathbb{R} \text { and } \eta \in X,
$$

it follows that $B(T(\theta) \xi, \rho)=T(\theta) B(\xi, \rho)$. Hence

$$
\Theta(\xi)_{\rho}=\cup_{\theta \in \mathbb{R}} T(\theta) B(\xi, \rho)
$$

and

$$
T(\theta) \Theta(\xi)_{\rho}=\Theta(\xi)_{\rho} \text { for all } \theta \in \mathbb{R} .
$$

In Section 4, we shall use the following property of orbits $\Theta(\xi) \neq\{\xi\}$.

Lemma 2.1. Suppose that $A \xi \neq 0$. Then there exists $R(\xi)>0$ such that, for any $\rho \in(0, R(\xi))$ and for any $v \in \Theta(\xi)_{\rho}$, there exists $\theta_{1} \in \mathbb{R}$ such that $\left\|v-T\left(\theta_{1}\right) \xi\right\|_{X}<\rho$ and $\left\langle v-T\left(\theta_{1}\right) \xi, A T\left(\theta_{1}\right) \xi\right\rangle_{X}=0$.

Remark. Clearly $\left\|v-T\left(\theta_{1}\right) \xi\right\|_{X} \geq d(v, \Theta(\xi))$ but we do not claim that $\left\|v-T\left(\theta_{1}\right) \xi\right\|_{X}=d(v, \Theta(\xi))$. In fact, if $\Theta(\xi)$ is not closed and $v \in \overline{\Theta(\xi)} \backslash \Theta(\xi)$, then $\|v-T(\theta) \xi\|_{X}>0=d(v, \Theta(\xi))$ for all $\theta \in \mathbb{R}$.

Proof. Since $T^{\prime}(0) \xi=A \xi \neq 0$, there exists $\psi>0$ such that $T(\psi) \xi \neq$ $T(0) \xi=\xi$ and $T(-\psi) \xi \neq T(0) \xi=\xi$. Let

$$
R(\xi)=\frac{1}{2} \min \left\{\|\xi-T(\psi) \xi\|_{X},\|\xi-T(-\psi) \xi\|_{X}\right\} .
$$

For $\rho \in(0, R(\xi))$ and $v \in \Theta(\xi)_{\rho}$, there exists some $\theta_{0}$ such that $T\left(\theta_{0}\right) \xi \in$ $B(v, \rho)$. Then we have that

$$
\begin{aligned}
\left\|v-T\left(\theta_{0} \pm \psi\right) \xi\right\|_{X} & \geq\left\|T\left(\theta_{0}\right) \xi-T\left(\theta_{0} \pm \psi\right) \xi\right\|_{X}-\left\|v-T\left(\theta_{0}\right) \xi\right\|_{X} \\
& =\|\xi-T( \pm \psi) \xi\|_{X}-\left\|v-T\left(\theta_{0}\right) \xi\right\|_{X} \\
& \geq 2 R(\xi)-\rho>\rho .
\end{aligned}
$$


Let $(p, q)$ be the maximal interval containing $\theta_{0}$ such that $T(\theta) \xi \in B(v, \rho)$ for all $\theta \in(p, q)$. Then $\theta_{0}-\psi<p<\theta_{0}<q<\theta_{0}+\psi$ and $\|v-T(p) \xi\|_{X}=$ $\|v-T(q) \xi\|_{X}=\rho$. Setting $f(\theta)=\|v-T(\theta) \xi\|_{X}^{2}$, there exists $\theta_{1} \in[p, q]$ such that $f\left(\theta_{1}\right)=\min \{f(\theta): p \leq \theta \leq q\} \leq f\left(\theta_{0}\right)<\rho^{2}$. Thus $\theta_{1} \in(p, q)$ and $f^{\prime}\left(\theta_{1}\right)=0$. Hence $\left\|v-T\left(\theta_{1}\right) \xi\right\|_{X}<\rho$ and

$$
0=\left\langle v-T\left(\theta_{1}\right) \xi, T^{\prime}\left(\theta_{1}\right) \xi\right\rangle=\left\langle v-T\left(\theta_{1}\right) \xi, A T\left(\theta_{1}\right) \xi\right\rangle .
$$

\subsection{Well-posedness of IVP}

The orbital stability of a standing wave requires that (IVP) to be globally well-posed in a neighbourhood of this solution. Let us make a few comments about this in our context.

Under the hypotheses (H1) to (H4), we consider that (IVP) is locally well-posed when the following condition is satisfied.

(LWP) For every $\xi \in X$ there exist $b_{+}, b_{-}>0$ and a unique function $u \in C\left(\left(-b_{-}, b_{+}\right), X\right)$ with $\Im u \in C^{1}\left(\left(-b_{-}, b_{+}\right), X^{*}\right)$ such that $u(0)=\xi$ and (2.1) is satisfied on $\left(-b_{-}, b_{+}\right)$. Furthermore, we require that

$$
H(u(t))=H\left(u_{0}\right) \text { and } Q(u(t))=Q\left(u_{0}\right) \text { for all } t \in\left(-b_{-}, b_{+}\right) .
$$

Remark 1. When (LWP) holds, let $\left(-b_{-}(\xi), b_{+}(\xi)\right)$ denote the maximal interval of existence for the solution with initial condition $\xi$.

Remark 2. If $u \in C^{1}\left(\left(-b_{-}, b_{+}\right), X\right)$, as is the case when $\operatorname{dim} X<\infty$, then $\frac{d}{d t}(\Im u)=\Im u^{\prime}$ and so

$$
\begin{aligned}
\frac{d}{d t} H(u(t)) & =\left\langle H^{\prime}(u(t)), u^{\prime}(t)\right\rangle=-\left\langle J^{*} \frac{d}{d t}(\Im u), u^{\prime}(t)\right\rangle \\
& =-\left\langle\Im \mathfrak{I} u^{\prime}(t), J u^{\prime}(t)\right\rangle=-\left(u^{\prime}(t), J u^{\prime}(t)\right)=0,
\end{aligned}
$$

and

$$
\begin{aligned}
\frac{d}{d t} Q(u(t)) & =\left\langle Q^{\prime}(u(t)), u^{\prime}(t)\right\rangle=\left\langle B u(t), u^{\prime}(t)\right\rangle \\
& =\left\langle J^{*} A^{*} \Im u(t), u^{\prime}(t)\right\rangle=\left\langle\Im u(t), A J u^{\prime}(t)\right\rangle \\
& =\left(u(t), A J u^{\prime}(t)\right)=\left(J A u(t), u^{\prime}(t)\right) \\
& =\left\langle\Im u^{\prime}(t), J A u(t)\right\rangle=\left\langle J^{*} H^{\prime}(u(t)), J A u(t)\right\rangle \\
& =\left\langle H^{\prime}(u(t)), J^{2} A u(t)\right\rangle=-\left\langle H^{\prime}(u(t)), A u(t)\right\rangle \\
& =0,
\end{aligned}
$$

showing that the conservation of $H$ and $Q$ along trajectories is a consequence of our assumptions of invariance in this case. In particular, when $\operatorname{dim} X<\infty$, (LWP) follows from our hypotheses (H1) to (H4) and the 
classical Picard Theorem for (1.1)

Remark 3. Since $H$ and $Q \in C(X, \mathbb{R})$, it follows from Remark 2 that (LWP) holds under the following three conditions.

(a) For every $\xi \in X$ there exist $b_{+}, b_{-}>0$ and a unique function $u \in$ $C\left(\left(-b_{-}, b_{+}\right), X\right)$ with $\mathfrak{I} u \in C^{1}\left(\left(-b_{-}, b_{+}\right), X^{*}\right)$ such that $u(0)=\xi$ and $(2.1)$ is satisfied on $\left(-b_{-}, b_{+}\right)$.

(b) The solution $u$ depends continuously on the initial condition $\xi$, in the sense that, if $\xi_{n} \rightarrow \xi$ in $X$ and $[p, q]$ is a compact sub-interval of $\left(-b_{-}(\xi), b_{+}(\xi)\right)$, then $[p, q] \subset\left(-b_{-}\left(\xi_{n}\right), b_{+}\left(\xi_{n}\right)\right)$ for all large $n$ and $u_{n}(t) \rightarrow$ $u(t)$ in $X$ as $n \rightarrow \infty$, for all $t \in[p, q]$.

(c) There is a dense subset $Y$ of $X$ such that, for all $\xi \in Y$, the solution $u$ with initial condition $\xi$ is smooth, in the sense that $u \in C^{1}\left(\left(-b_{-}, b_{+}\right), X\right)$.

We consider that (IVP) is globally well-posed at $\xi \in X$ when the following condition is satisfied.

(GWP at $\xi$ ) The condition (LWP) is satisfied and there exists an open neighbourhood $\Omega$ of $\xi$ in $X$ such that $b_{+}\left(u_{0}\right)=\infty$ for all $u_{0} \in \Omega$.

The conserved quantities can help to ensure that (IVP) is globally well-posed. In this direction we shall use several variants of the following compactness condition.

$(\mathrm{CC})_{c, d} H^{\prime}: X \rightarrow X^{*}$ is bounded on bounded subsets of $X$ and there exists $\varepsilon>0$ such that, for any $h \in(c-\varepsilon, c+\varepsilon)$ and $q \in(d-\varepsilon, d+\varepsilon)$,

$$
\{v \in X: H(v)=h\} \cap\{v \in X: Q(v)=q\}
$$

is a compact subset of $X$.

Remark. If $\operatorname{dim} X<\infty$, then $(\mathrm{CC})_{c, d}$ is satisfied provided that $\{v \in X: H(v)=h\} \cap\{v \in X: Q(v)=q\}$ is bounded for all $h$ near $c$ and all $q$ near $d$.

Proposition 2.2. Let (LWP) hold and suppose that $\xi \in X$ is such that $(C C)_{H(\xi), Q(\xi)}$ is satisfied. Then (GWP at $\left.\xi\right)$ holds.

Proof. Let $\varepsilon>0$ be given by $(\mathrm{CC})_{H(\xi), Q(\xi)}$. Then there exists $\delta>0$ such that $|H(v)-H(\xi)|<\varepsilon$ and $|Q(v)-Q(\xi)|<\varepsilon$ for all $v \in B(\xi, \delta)$. We claim that $b_{+}\left(u_{0}\right)=\infty$ for all $u_{0} \in B(\xi, \delta)$.

To prove this we consider $u_{0} \in B(\xi, \delta)$ and a sequence $\left\{t_{n}\right\} \subset\left(0, b_{+}\left(u_{0}\right)\right)$ such that $t_{n} \rightarrow b_{+}\left(u_{0}\right)$. Since

$$
H\left(u\left(t_{n}\right)\right)=H\left(u_{0}\right) \text { and } Q\left(u\left(t_{n}\right)\right)=Q\left(u_{0}\right)
$$


it follows from $(\mathrm{CC})_{H(\xi), Q(\xi)}$ that there exist $w \in X$ and a subsequence such that $u\left(t_{n_{k}}\right) \rightarrow w$ in $X$. From this it follows easily that $\left\{\|u(t)\|_{X}: 0 \leq\right.$ $\left.t<b_{+}\left(u_{0}\right)\right\}$ is bounded and hence so is

$$
\left\{\left\|H^{\prime}(u(t))\right\|_{X^{*}}: 0 \leq t<b_{+}\left(u_{0}\right)\right\} .
$$

Let us suppose that $b_{+}\left(u_{0}\right)<\infty$.

For $0 \leq s<t<b_{+}\left(u_{0}\right)$, we have that

$$
\Im u(t)-\Im u(s)=\int_{s}^{t} J^{*} H^{\prime}(u(\tau)) d \tau
$$

and so

$$
\Im w-\Im u(s)=\lim _{t_{n_{k}} \rightarrow b_{+}\left(u_{0}\right)} \int_{s}^{t_{n_{k}}} J^{*} H^{\prime}(u(\tau)) d \tau .
$$

But

$$
\left\|\int_{s}^{t_{n_{k}}} J^{*} H^{\prime}(u(\tau)) d \tau\right\|_{X^{*}} \leq \sup _{0 \leq \tau<b_{+}\left(u_{0}\right)}\left\|H^{\prime}(u(\tau))\right\|\left|t_{n_{k}}-s\right|
$$

and so

$$
\|\Im w-\Im u(s)\|_{X^{*}} \leq \sup _{0 \leq \tau<b_{+}\left(u_{0}\right)}\left\|H^{\prime}(u(\tau))\right\|\left|b_{+}\left(u_{0}\right)-s\right|,
$$

showing that $\|\mathfrak{I} w-\mathfrak{I} u(s)\|_{X^{*}} \rightarrow 0$ as $s \rightarrow b_{+}\left(u_{0}\right)$.

In fact, we can deduce that $\|w-u(s)\|_{X} \rightarrow 0$ as $s \rightarrow b_{+}\left(u_{0}\right)$. Otherwise, there exist $\eta>0$ and a sequence $\left\{s_{n}\right\} \subset\left(0, b_{+}\left(u_{0}\right)\right)$ such that $s_{n} \rightarrow b_{+}\left(u_{0}\right)$ and $\left\|w-u\left(s_{n}\right)\right\|_{X} \geq \eta$ for all $n \in \mathbb{N}$. But then, as before, $(\mathrm{CC})_{H(\xi), Q(\xi)}$ implies that there exist $z \in X$ and a subsequence such that $u\left(s_{n_{k}}\right) \rightarrow z$ in $X$. From which it follows that $\mathfrak{I} u\left(s_{n_{k}}\right) \rightarrow \mathfrak{I} z$ in $X^{*}$. However, we also have that $\mathfrak{I} u\left(s_{n_{k}}\right) \rightarrow \mathfrak{I} w$ in $X^{*}$ and so $z=w$ by the injectivity of $\mathfrak{I}: X \rightarrow X^{*}$. Thus $\left\|w-u\left(s_{n_{k}}\right)\right\|_{X} \rightarrow 0$ and we have a contradiction. Hence $\|w-u(s)\|_{X} \rightarrow 0$ as $s \rightarrow b_{+}\left(u_{0}\right)$.

Since (2.1) is autonomous, it follows from (LWP) that there is a unique function $z \in C\left(\left(-c_{-}, c_{+}\right), X\right)$ with $\mathfrak{I} z \in C^{1}\left(\left(-c_{-}, c_{+}\right), X^{*}\right)$ such that

$$
\begin{aligned}
\frac{d}{d t} \Im z & =J^{*} H^{\prime}(z(t)) \text { for } t \in\left(-c_{-}, c_{+}\right) \\
z\left(b_{+}\left(u_{0}\right)\right) & =w
\end{aligned}
$$

where $c_{-}=-b_{-}(w)+b_{+}\left(u_{0}\right)$ and $c_{+}=b_{+}(w)+b_{+}\left(u_{0}\right)$.

Now consider the function $v:\left(-b_{-}\left(u_{0}\right), c_{+}\right) \rightarrow X$ defined by

$$
v(t)=\left\{\begin{array}{lc}
u(t) & \text { for } t \in\left(-b_{-}\left(u_{0}\right), b_{+}\left(u_{0}\right)\right) \\
z(t) & \text { for } t \in\left[b_{+}\left(u_{0}\right), b_{+}(w)+b_{+}\left(u_{0}\right)\right)
\end{array} .\right.
$$


We have already proved that $v \in C\left(\left(-b_{-}\left(u_{0}\right), b_{+}(w)+b_{+}\left(u_{0}\right)\right), X\right)$ and

$$
\begin{aligned}
\mathfrak{I} w-\mathfrak{I} v(s) & =\mathfrak{I} w-\mathfrak{I} u(s)=\int_{s}^{b_{+}(w)} J^{*} H^{\prime}(u(\tau)) d \tau \\
& =\int_{s}^{b_{+}(w)} J^{*} H^{\prime}(v(\tau)) d \tau \text { for }-b_{-}\left(u_{0}\right)<s<b_{+}\left(u_{0}\right), \\
\mathfrak{I} w-\mathfrak{I} v(s) & =\mathfrak{I} w-\mathfrak{I} z(s)=-\int_{b_{+}(w)}^{s} J^{*} H^{\prime}(z(\tau)) d \tau \\
& =-\int_{b_{+}(w)}^{s} J^{*} H^{\prime}(v(\tau)) d \tau \text { for } b_{+}\left(u_{0}\right)<s<b_{+}(w)+b_{+}\left(u_{0}\right) .
\end{aligned}
$$

It follows that $\mathfrak{I} v \in C^{1}\left(\left(-b_{-}\left(u_{0}\right), b_{+}(w)+b_{+}\left(u_{0}\right)\right), X^{*}\right)$ with

$$
\frac{d}{d t} \Im v(t)=J^{*} H^{\prime}(v(t)) \text { for all } t \in\left(-b_{-}\left(u_{0}\right), b_{+}(w)+b_{+}\left(u_{0}\right)\right) \text {. }
$$

This implies that $-c_{-} \leq-b_{-}\left(u_{0}\right)$ and that $z(t)=v(t)$ for all $t \in$ $\left(-b_{-}\left(u_{0}\right), b_{+}(w)+b_{+}\left(u_{0}\right)\right)$. Since $b_{+}(w)>0$ this contradicts the maximality of the interval

$\left(-b_{-}\left(u_{0}\right), b_{+}\left(u_{0}\right)\right)$ for the solution $u$ with initial condition $u(0)=u_{0}$. Hence $b_{+}\left(u_{0}\right)=\infty$, justifying our claim.

\subsection{Summary}

We introduced the basic hypotheses (H1) to (H4) progressively in order to show their utility. Now, for future reference, we regroup them in a more compact, equivalent form.

In a standard variational triple $X \subset H=H^{*} \subset X^{*}$ with

$$
\langle R u, v\rangle=\langle u, v\rangle_{X} \text { and }\langle\Im \mathfrak{I} u, v\rangle=(u, v) \text { for all } u, v \in X,
$$

we introduce the following operators.

(H) (i) $J \in B(X, X)$ with $J^{2}=-I$ and

$\langle J v, w\rangle_{X}=-\langle v, J w\rangle_{X}$ and $(J v, w)=-(v, J w)$ for all $v, w \in X$.

(ii) $A \in B(X, X)$ with $A J=J A$ and

$\langle A v, w\rangle_{X}=-\langle v, A w\rangle_{X}$ and $(A v, w)=-(v, A w)$ for all $v, w \in X$.

(iii) $H \in C^{2}(X, \mathbb{R})$ and

$$
\left\langle H^{\prime}(v), A v\right\rangle=0 \text { for all } v \in X \text {. }
$$

Remarks. Clearly (i) implies that $J: X \rightarrow X$ is an isomorphism and, putting $w=J v$, we see that

$$
\|J v\|_{X}=\|v\|_{X} \text { and }\|J v\|=\|v\| \text { for all } v \in X .
$$


Recalling that $S=R^{-1} \mathfrak{I} \in B(X, X)$ with $S^{T}=S$ and

$$
(v, w)=\langle S v, w\rangle_{X} \text { for all } v, w \in X,
$$

we see that (i) is equivalent to

(i') $J \in B(X, X)$ with $J=-J^{-1}=-J^{T}$ and $J S=S J$.

It implies that $J$ induces two symplectic forms on $X$ :

$$
\omega_{X}(v, w)=\langle J v, w\rangle_{X} \text { and } \omega_{H}(v, w)=(J v, w) \text { for } v, w \in X .
$$

In the same way, the condition (ii) is equivalent to

$$
\text { (ii') } A \in B(X, X) \text { with } A=-A^{T} \text { and } A S=S A \text {. }
$$

It implies that $T(\theta)=e^{\theta A}$ is an isometry both for $\|\cdot\|_{X}$ and $\|\cdot\|$.

Taken together, (i) and (ii) imply that $\Gamma=\{T(\theta): \theta \in \mathbb{R}\}$ preserves both symplectic forms:

$$
\omega_{X}(g v, g w)=\omega_{X}(v, w) \text { and } \omega_{H}(g v, g w)=\omega_{H}(v, w) \text { for all } g \in \Gamma .
$$

The condition (iii) implies that $H$ is $\Gamma$-invariant. As we show below, a consequence of this invariance is that $\left\langle H^{\prime}(0), A w\right\rangle=0$ for all $w \in X$ and hence, if $A X$ is dense in $X$, that $H^{\prime}(0)=0$.

Noting that $(\mathrm{H})$ is equivalent to $(\mathrm{H} 1)$ to $(\mathrm{H} 4)$, we consider henceforth the Hamiltonian system,

$$
\frac{d}{d t}(\Im u)=J^{*} H^{\prime}(u) \text { in } X^{*}
$$

under the tacit assumption that $(\mathrm{H})$ holds.

When $(H,(\cdot, \cdot),\|\cdot\|)=\left(X,\langle\cdot, \cdot\rangle_{X},\|\cdot\|_{X}\right)$, it follows that the Hamiltonian $H$ and the function $Q(v)=\frac{1}{2} \omega_{H}(A v, v)$ are constant on solutions of (IVP). These quantities are also conserved in more general situations, see Remarks 2 and 3 in Section 2.6.

A standing wave is a special solution of the form

$$
u(t)=e^{\lambda t A} \xi \text { where } \lambda \in \mathbb{R} \text { and } \xi \in X .
$$

We consider the existence and orbital stability of non-stationary (i.e. $\lambda A \xi \neq$ $0)$ standing waves. They are generated by solutions of the stationary equation $\left(H^{\prime}(\xi)=\lambda B(\xi)\right.$ where $\left.B=J^{*} A^{*} \mathfrak{I}=\mathfrak{I} A J\right)$ which can also be written as $D_{v} G_{\lambda}(\xi)=0$ where $G_{\lambda}=H-\lambda Q \in C^{2}(X, \mathbb{R})$ is the augmented Hamiltonian and $Q(v)=\frac{1}{2}\langle B v, v\rangle=\frac{1}{2}(v, A J v)=\frac{1}{2} \omega_{H}(A v, v)$. The function $Q$ is $\Gamma$-invariant and hence so is $G_{\lambda}$. 
A particularly simple situation occurs when $H \in C^{2}(X, \mathbb{R})$ and $\left\langle H^{\prime}(v), J v\right\rangle=0$ for all $v \in X$ for some operator $J$ satisfying $(\mathrm{H})(\mathrm{i})$. Then we can choose $A=J$ (or $A=-J$ ) and we have that (H)(i)-(iii) holds with $H^{\prime}(0)=0$. The nonlinear Schrödinger equation provides an example of this case.

\section{Consequences of the invariance}

By the $\Gamma$-invariance of $G_{\lambda}$, for all $v \in X$ and $\theta \in \mathbb{R}$,

$G_{\lambda}(T(\theta) v)=G_{\lambda}(v)$

$\left\langle D_{u} G_{\lambda}(T(\theta) v), T(\theta) w\right\rangle=\left\langle D_{u} G_{\lambda}(v), w\right\rangle$ for all $w \in X$,

$\left\langle D_{u} G_{\lambda}(T(\theta) v), T^{\prime}(\theta) v\right\rangle=0$

$\left\langle D_{u u}^{2} G_{\lambda}(T(\theta) v) T(\theta) z, T(\theta) w\right\rangle=\left\langle D_{u u}^{2} G_{\lambda}(v) z, w\right\rangle$ for all $w, z \in X$,

$\left\langle D_{u u}^{2} G_{\lambda}(T(\theta) v) T^{\prime}(\theta) v, T(\theta) w\right\rangle+\left\langle D_{u} G_{\lambda}(T(\theta) v), T^{\prime}(\theta) w\right\rangle=0$

showing that

$$
\begin{aligned}
& D_{u} G_{\lambda}(T(\theta) v)=T(-\theta)^{*} D_{u} G_{\lambda}(v), \quad\left\langle D_{u} G_{\lambda}(v), A v\right\rangle=0, \\
& D_{u u}^{2} G_{\lambda}(T(\theta) v) T(\theta)=T(-\theta)^{*} D_{u u}^{2} G_{\lambda}(v), \\
& D_{u u}^{2} G_{\lambda}(v) A v=-A^{*} D_{u} G_{\lambda}(v)
\end{aligned}
$$

for all $v \in X$ and $\theta \in \mathbb{R}$.

Observe that all of these identities remain true when $G_{\lambda}$ is replaced by $H$.

If $D_{u} G_{\lambda}\left(\xi_{\lambda}\right)=0$, then

$$
D_{u} G_{\lambda}\left(T(\theta) \xi_{\lambda}\right)=0 \text { and } T(\theta)^{*} D_{u u}^{2} G_{\lambda}\left(T(\theta) \xi_{\lambda}\right) T^{\prime}(\theta) \xi_{\lambda}=0
$$

for all $\theta \in \mathbb{R}$. In particular,

$$
D_{u} G_{\lambda}\left(\xi_{\lambda}\right)=0 \text { implies that } D_{u u}^{2} G_{\lambda}\left(\xi_{\lambda}\right) A \xi_{\lambda}=0,
$$

so $\xi_{\lambda}$ is a degenerate critical point of $G_{\lambda}$ whenever $A \xi_{\lambda} \neq 0$.

The stationary solution $u=0$

If $H^{\prime}(0)=0$ we have that $u(t) \equiv 0$ is a stationary solution of $(2.1)$. Recall that this always occurs when $A X$ is dense in $X$. The linearization of $(2.1)$ at $u \equiv 0$ is

$$
\frac{d}{d t}(\Im v)(t)=J^{*} H^{\prime \prime}(0) v(t)
$$

which is a linear autonomous Hamiltonian system. From the invariance properties listed above, we see that $H^{\prime \prime}(0) T(\theta)=T(-\theta)^{*} H^{\prime \prime}(0)$ for all 
$\theta \in \mathbb{R}$ and so $H^{\prime \prime}(0) A=-A^{*} H^{\prime \prime}(0)$. In the special case mentioned above where $A= \pm J$, this means that $J^{*} H^{\prime \prime}(0)$ is skew-symmetric and hence the stability of the stationary solution $u \equiv 0$ cannot be deduced from the stability of the linearization (2.5). For example, in the case where $(H,(\cdot, \cdot),\|\cdot\|)=\left(X,\langle\cdot, \cdot\rangle_{X},\|\cdot\|_{X}\right)$ and $A= \pm J$, we have that $J^{*}=J^{T}$ and

$$
\left[J^{T} H^{\prime \prime}(0)\right]^{T}=H^{\prime \prime}(0)^{T} J=H^{\prime \prime}(0) J=-J^{T} H^{\prime \prime}(0),
$$

showing that $J^{T} H^{\prime \prime}(0): X \rightarrow X$ is skew-symmetric and hence that its spectrum is a subset of the imaginary axis.

\section{A canonical form in finite dimensions}

When $(H,(\cdot, \cdot),\|\cdot\|)=\left(X,\langle\cdot, \cdot\rangle_{X},\|\cdot\|_{X}\right)$, the condition $(\mathrm{H})$ reduces to

$$
\begin{aligned}
J^{T} & =J^{-1}=-J, A=-A^{T}, A J=J A, \\
H & \in C^{2}(X, \mathbb{R}) \text { and }\left\langle H^{\prime}(v), A v\right\rangle=0 \text { for all } v \in X .
\end{aligned}
$$

Setting $B=J A$, we have that $B=B^{T}$ and $B J=J B$. Suppose, in addition, that $\operatorname{dim} X<\infty$. Then (LWP) is implied by (H). Furthermore, if $\mu \in \mathbb{R}$ is an eigenvalue of $B$ with eigenvector $\varphi$, then $B J \varphi=\mu J \varphi$ and $\langle J \varphi, \varphi\rangle=0$. Hence all eigenvalues of $B$ have even multiplicity and $X$ has an orthonormal basis of the form

$$
\begin{gathered}
\left\{\varphi_{1}, J \varphi_{1}, \varphi_{2}, J \varphi_{2}, \ldots, \varphi_{N}, J \varphi_{N}\right\} \\
\text { with } B \varphi_{j}=\mu_{j} \varphi_{j} \text { and } B J \varphi_{j}=\mu_{j} J \varphi_{j} \text { for } j=1, \ldots, N .
\end{gathered}
$$

Noting that $A J=J A$, we see that $\left\{\varphi_{j}, J \varphi_{j}\right\}$ is also an invariant subspace for $A$ with $A \varphi_{j}=-\mu_{j} J \varphi_{j}$ and $A J \varphi_{j}=\mu_{j} \varphi_{j}$ for $j=1, \ldots, N$. With respect to this basis the operators have the following $2 N \times 2 N$-matrix representations

$$
\begin{aligned}
& B=\operatorname{diag}\left[\begin{array}{cc}
\mu_{j} & 0 \\
0 & \mu_{j}
\end{array}\right], J=\operatorname{diag}\left[\begin{array}{cc}
0 & 1 \\
-1 & 0
\end{array}\right], A=\operatorname{diag}\left[\begin{array}{cc}
0 & -\mu_{j} \\
\mu_{j} & 0
\end{array}\right] \\
& \text { and } T(\theta)=\operatorname{diag}\left[\begin{array}{cc}
\cos \mu_{j} \theta & -\sin \mu_{j} \theta \\
\sin \mu_{j} \theta & \cos \mu_{j} \theta
\end{array}\right],
\end{aligned}
$$

showing that $T$ is either periodic or quasi-periodic depending on whether or not the eigenvalues of $B$ are rationally dependent. In the first case, the non-trivial orbits are closed curves whereas in the latter case, the closure of the orbit in $X$ contains a $k$-dimensional torus for some $k \in\{2, N\}$. 


\section{Sufficient conditions for the orbital stability of a standing wave}

We state immediately the main result giving sufficient conditions for the orbital stability of a standing wave. Then we derive some useful alternative formulations of it.

Theorem 3.1. Suppose that $\left(\lambda, \xi_{\lambda}\right) \in \mathbb{R} \times X$ is such that

$$
D_{v} G_{\lambda}\left(\xi_{\lambda}\right)=0 \text { with } \lambda A \xi_{\lambda} \neq 0
$$

and $\left(G W P\right.$ at $\left.\xi_{\lambda}\right)$ is satisfied. Then the standing wave $u_{\lambda}(t)=T(\lambda t) \xi_{\lambda}$ generated by $\xi_{\lambda}$ is orbitally stable provided that:

there exists $\delta>0$ such that

$$
\left\langle D_{v v}^{2} G_{\lambda}\left(\xi_{\lambda}\right) z, z\right\rangle \geq \delta\|z\|_{X}^{2} \text { for all } z \in\left\{A \xi_{\lambda}, R^{-1} B \xi_{\lambda}\right\}^{\perp},
$$

where $\left\{A \xi_{\lambda}, R^{-1} B \xi_{\lambda}\right\}^{\perp}=\left\{z \in X:\left\langle z, A \xi_{\lambda}\right\rangle_{X}=\left\langle z, R^{-1} B \xi_{\lambda}\right\rangle_{X}=0\right\}$.

The proof of this result will be given in the next section.

Remarks. First we recall that $A \xi_{\lambda}$ is tangent to the orbit $\Theta\left(\xi_{\lambda}\right)$ at $\xi_{\lambda}$ and that $D_{v v}^{2} G_{\lambda}\left(\xi_{\lambda}\right) A \xi_{\lambda}=0$. Hence $D_{v v}^{2} G_{\lambda}\left(\xi_{\lambda}\right)$ can never be positive definite on $X$. If

$$
\left\langle D_{v v}^{2} G_{\lambda}\left(\xi_{\lambda}\right) z, z\right\rangle \geq \delta\|z\|_{X}^{2} \text { for all } z \in\left\{A \xi_{\lambda}\right\}^{\perp},
$$

then (SC) holds. But (SC) allows $D_{v v}^{2} G_{\lambda}\left(\xi_{\lambda}\right) \leq 0$ on a subspace of dimension 2. In fact, for any $v \in X$,

$$
\left\langle R^{-1} B \xi_{\lambda}, v\right\rangle_{X}=\left\langle B \xi_{\lambda}, v\right\rangle=\left(\xi_{\lambda}, A J v\right)=\left(J A \xi_{\lambda}, v\right) .
$$

Thus

$$
\left\langle R^{-1} B \xi_{\lambda}, J A \xi_{\lambda}\right\rangle_{X}=\left\|J A \xi_{\lambda}\right\|^{2}=\left\|A \xi_{\lambda}\right\|^{2}>0,
$$

so $R^{-1} B \xi_{\lambda} \neq 0$ and, in addition,

$$
\left\langle R^{-1} B \xi_{\lambda}, A \xi_{\lambda}\right\rangle_{X}=\left(J A \xi_{\lambda}, A \xi_{\lambda}\right)=0 .
$$

Thus the space $\left\{A \xi_{\lambda}, R^{-1} B \xi_{\lambda}\right\}^{\perp}$ has codimension 2 in $X$.

The orthogonality conditions in (SC) are

$$
\begin{aligned}
\left\langle z, A \xi_{\lambda}\right\rangle_{X} & =\left\langle z, R^{-1} B \xi_{\lambda}\right\rangle_{X}=0 \text { where } \\
\left\langle z, R^{-1} B \xi_{\lambda}\right\rangle_{X} & =\left\langle B \xi_{\lambda}, z\right\rangle=\left(\xi_{\lambda}, A J z\right)=\left(z, J A \xi_{\lambda}\right) .
\end{aligned}
$$

The condition (SC) can be replaced by the following variant using only orthogonality with respect to $(\cdot, \cdot)$. 
Lemma 3.2. Suppose that there exists $\delta>0$ such that

$$
\left\langle D_{v v}^{2} G_{\lambda}\left(\xi_{\lambda}\right) v, v\right\rangle \geq \delta\|v\|_{X}^{2} \text { for all } v \in X \text { with }\left(v, A \xi_{\lambda}\right)=\left(v, J A \xi_{\lambda}\right)=0 \text {. }
$$

Then the condition (SC) is satisfied.

Consequently the conditions (3.1), (GWP at $\left.\xi_{\lambda}\right)$ and $\left(S C^{*}\right)$ imply that the standing wave $u(t)=T(\lambda t) \xi_{\lambda}$ is orbitally stable.

Remark. In dealing with conditions $(\mathrm{SC})$ and $\left(\mathrm{SC}^{*}\right)$, it is sometimes convenient to set

$$
S_{\lambda}=R^{-1} D_{v v}^{2} G_{\lambda}\left(\xi_{\lambda}\right) .
$$

Then $S_{\lambda}: X \rightarrow X$ is a bounded self-adjoint operator and (SC) reads

$$
\left\langle S_{\lambda} z, z\right\rangle_{X} \geq \delta\|z\|_{X}^{2} \text { for all } z \in\left\{A \xi_{\lambda}, R^{-1} B \xi_{\lambda}\right\}^{\perp} .
$$

Recall that $S_{\lambda} A \xi_{\lambda}=0$.

Proof. Let $\varphi=A \xi_{\lambda} /\left\|A \xi_{\lambda}\right\|$ and $\psi=J \varphi$, so that

$$
\|\varphi\|=\|\psi\|=1 \text { and }(\varphi, \psi)=0 \text {. }
$$

Now consider any $z \in\left\{A \xi_{\lambda}, R^{-1} B \xi_{\lambda}\right\}^{\perp}$. That is

$$
z \in X \text { and }\left\langle z, A \xi_{\lambda}\right\rangle_{X}=\left\langle z, R^{-1} B \xi_{\lambda}\right\rangle_{X}=0 .
$$

Setting

$$
v=z-(z, \varphi) \varphi-(z, \psi) \psi,
$$

we have that $(v, \varphi)=(v, \psi)=0$ and so, by $\left(\mathrm{SC}^{*}\right)$,

$$
\left\langle S_{\lambda} v, v\right\rangle_{X} \geq \delta\|v\|_{X}^{2} .
$$

But $(z, \psi)=\left(z, J A \xi_{\lambda}\right) /\left\|A \xi_{\lambda}\right\|=\left\langle z, R^{-1} B \xi_{\lambda}\right\rangle_{X} /\left\|A \xi_{\lambda}\right\|=0$ and $S_{\lambda} \varphi=0$, showing that

$$
v=z-(z, \varphi) \varphi \text { and } S_{\lambda} v=S_{\lambda} z .
$$

Hence

$$
\left\langle S_{\lambda} z, z\right\rangle_{X}=\left\langle S_{\lambda} v, v+(z, \varphi) \varphi\right\rangle_{X}=\left\langle S_{\lambda} v, v\right\rangle_{X}
$$

and

$$
\|z\|_{X}^{2}=\langle z, v+(z, \varphi) \varphi\rangle_{X}=\langle z, v\rangle_{X} \text {, since }\langle z, \varphi\rangle_{X}=0 .
$$

Thus $\|z\|_{X}^{2} \leq\|z\|_{X}\|v\|_{X}$ and we have that

$$
\left\langle S_{\lambda} z, z\right\rangle_{X} \geq\left\langle S_{\lambda} v, v\right\rangle_{X} \geq \delta\|v\|_{X}^{2} \geq \delta\|z\|_{X}^{2},
$$

showing that (SC) holds. 
Continuing further in the direction suggested by $\left(\mathrm{SC}^{*}\right)$, we can formulate the stability condition using the unbounded self-adjoint operator acting in $H$ which is associated with the Hessian of the augmented Hamiltonian. Suppose that it is coercive in the following sense:

(G) There exist $\varepsilon, C>0$ such that

$$
\left\langle D_{v v}^{2} G_{\lambda}\left(\xi_{\lambda}\right) v, v\right\rangle \geq \varepsilon\|v\|_{X}^{2}-C\|v\|^{2} \text { for all } v \in X
$$

For elliptic operators, this is Gårding's inequality.

Lemma 3.3. Suppose that $D_{v v}^{2} G_{\lambda}\left(\xi_{\lambda}\right)$ satisfies $(G)$ and define a quadratic form $b_{\lambda}: D\left(b_{\lambda}\right) \subset H \rightarrow \mathbb{R}$ by

$$
b_{\lambda}(v)=\left\langle D_{v v}^{2} G_{\lambda}\left(\xi_{\lambda}\right) v, v\right\rangle \text { for all } v \in X=D\left(b_{\lambda}\right) .
$$

Then $b_{\lambda}$ is a closed, densely defined, form on $H$ that is bounded below. Consequently, there is a self-adjoint operator $L_{\lambda}: D\left(L_{\lambda}\right) \subset H \rightarrow H$ defined by

$$
\begin{aligned}
D\left(L_{\lambda}\right) & =\{z \in X: \text { there exists } w \in H \text { such that } \\
\left\langle D_{v v}^{2} G_{\lambda}\left(\xi_{\lambda}\right) z, v\right\rangle & =(w, v) \text { for all } v \in X\}, \\
L_{\lambda} z & =w \text { for all } z \in D\left(L_{\lambda}\right) .
\end{aligned}
$$

Remarks. $L_{\lambda}$ is the only self-adjoint operator $L$ acting in $H$ that has the properties

$$
D(L) \subset X \text { and }\left\langle D_{v v}^{2} G_{\lambda}\left(\xi_{\lambda}\right) z, v\right\rangle=(L z, v) \text { for all } z \in L \text { and } v \in X .
$$

Furthermore, $D\left(L_{\lambda}\right)$ is a core for $b_{\lambda}$ and so $D\left(L_{\lambda}\right)$ is dense in the Hilbert space $\left(X,\langle\cdot, \cdot\rangle_{X}\right)$. Note that $D_{v v}^{2} G_{\lambda}\left(\xi_{\lambda}\right) z=\mathfrak{I} L_{\lambda} z$ for $z \in D\left(L_{\lambda}\right)$.

Proof. To show that $b_{\lambda}$ is closed in $H$, we consider a sequence $\left\{v_{n}\right\}$ in $D\left(b_{\lambda}\right)=X$ such that $\left\|v_{n}-v\right\| \rightarrow 0$ and $b_{\lambda}\left(v_{n}-v_{m}\right) \rightarrow 0$ as $n, m \rightarrow \infty$, for some $v \in H$. The condition (G) then implies that $\left\|v_{n}-v_{m}\right\|_{X} \rightarrow 0$, showing that $\left\{v_{n}\right\}$ is a Cauchy sequence in $X$. Hence there exists $w \in X$ such that $\left\|v_{n}-w\right\|_{X} \rightarrow 0$. Since $X$ is continuously embedded in $H$ we must have $w=v$. Then the continuity of $D_{v v}^{2} G_{\lambda}\left(\xi_{\lambda}\right): X \rightarrow X^{*}$ shows that $b_{\lambda}\left(v_{n}-v\right) \rightarrow 0$, proving that $b_{\lambda}$ is closed. It is bounded below since $b_{\lambda}(v) \geq-C\|v\|^{2}$ for all $v \in X$, by $(\mathrm{G})$.

The existence and uniqueness of $L_{\lambda}$ now follow from the First Representation Theorem in Kato [21], Theorems 2.1 and 2.6 in Chapter VI. 
Corollary 3.4. Suppose that $\left(\lambda, \xi_{\lambda}\right) \in \mathbb{R} \times X$ is such that $(G)$ holds. Suppose that for $L_{\lambda}$ as defined in Lemma 3.3, we have that there exists $\delta>0$ such that

$$
\left(L_{\lambda} v, v\right) \geq \delta\|v\|^{2} \text { for all } v \in D\left(L_{\lambda}\right) \text { with }\left(v, A \xi_{\lambda}\right)=\left(v, J A \xi_{\lambda}\right)=0 .
$$

Then the condition $\left(\mathrm{SC}^{*}\right)$ is satisfied.

Consequently the conditions (3.1), (GWP at $\left.\xi_{\lambda}\right),(\mathrm{G})$ and $\left(\mathrm{SC}^{* *}\right)$ imply that the standing wave $u(t)=T(\lambda t) \xi_{\lambda}$ is orbitally stable.

Proof. By $\left(\mathrm{SC}^{* *}\right)$ we have that

$$
\left(L_{\lambda} v, v\right) \geq \delta\|v\|^{2} \text { for all } v \in D\left(L_{\lambda}\right) \text { with }\left(v, A \xi_{\lambda}\right)=\left(v, J A \xi_{\lambda}\right)=0
$$

and by $(\mathrm{G})$

$$
\left(L_{\lambda} v, v\right) \geq \varepsilon\|v\|_{X}^{2}-C\|v\|^{2} \text { for all } v \in X .
$$

Hence

$\left[1+\frac{\delta}{C}\right]\left(L_{\lambda} v, v\right) \geq \frac{\varepsilon \delta}{C}\|v\|_{X}^{2}$ for all $v \in D\left(L_{\lambda}\right)$ with $\left(v, A \xi_{\lambda}\right)=\left(v, J A \xi_{\lambda}\right)=0$.

Thus

$\left\langle D_{v v}^{2} G_{\lambda}\left(\xi_{\lambda}\right) v, v\right\rangle \geq \frac{\varepsilon \delta}{C+\delta}\|v\|_{X}^{2}$ for all $v \in D\left(L_{\lambda}\right)$ with $\left(v, A \xi_{\lambda}\right)=\left(v, J A \xi_{\lambda}\right)$.

The density of $D\left(L_{\lambda}\right)$ in $X$ for $\|\cdot\|_{X}$ now yields $\left(\mathrm{SC}^{*}\right)$ and the rest follows from Lemma 3.2.

\section{Proof of the main stability theorem}

We fix a value of $\lambda$ for which (3.1) and (SC) are satisfied. Theorem 3.1 is proved by constructing a $\Gamma$-invariant Lyapunov function $V$ for (IVP). This line of attack has a long history. See [34] and [14]. For a useful heuristic description, see [23].

Since Lyapunov functions are mostly used to prove the stability of stationary solutions, it is worth noting that we can make a change of variable so that the standing wave $u_{\lambda}$ becomes a stationary solution of a new $\Gamma$ invariant Hamiltonian system. Then $\Theta\left(\xi_{\lambda}\right)$ becomes an orbit (in the sense of the symmetry group) of stationary solutions of this new system and $V$ is a Lyapunov function for this new problem too. 


\subsection{Change of variable}

Given a function $u:[0, \infty) \rightarrow X$ we set $w(t)=T(-\lambda t) u(t)$ so that $u(t)=$ $T(\lambda t) w(t)$. Clearly $u \in C([0, \infty), X) \Longleftrightarrow w \in C([0, \infty), X)$. Furthermore for any $v \in X$,

$$
\begin{aligned}
\langle\Im u(t), v\rangle & =(u(t), v)=(T(\lambda t) w(t), v)=(w(t), T(-\lambda t) v) \\
& =\left\langle T(-\lambda t)^{*} \Im w(t), v\right\rangle
\end{aligned}
$$

so that

$$
\mathfrak{I} u(t)=T(-\lambda t)^{*} \mathfrak{I} w(t) \text { and } \mathfrak{I} w(t)=T(\lambda t)^{*} \mathfrak{I} u(t) .
$$

Recalling that $A^{*} \in B\left(X^{*}, X^{*}\right)$, we have that $T(\theta)^{*}=e^{\theta A^{*}}$ for all $\theta \in \mathbb{R}$. It now follows that $\Im u \in C^{1}\left((0, \infty), X^{*}\right) \Longleftrightarrow \Im w \in C^{1}\left((0, \infty), X^{*}\right)$ and

$$
\begin{aligned}
\frac{d}{d t} \Im u & =\frac{d}{d t}\left\{T(-\lambda t)^{*} \Im w(t)\right\} \\
& =-\lambda T^{\prime}(-\lambda t)^{*} \Im w(t)+T(-\lambda t)^{*} \frac{d}{d t} \Im w(t) \\
& =-\lambda T(-\lambda t)^{*} A^{*} \Im w(t)+T(-\lambda t)^{*} \frac{d}{d t} \Im w(t) \\
& =T(-\lambda t)^{*}\left\{-\lambda A^{*} \Im w(t)+\frac{d}{d t} \Im w(t)\right\} .
\end{aligned}
$$

Furthermore,

$$
H^{\prime}(u(t))=H^{\prime}(T(\lambda t) w(t))=T(-\lambda t)^{*} H^{\prime}(w(t))
$$

and $J^{*} H^{\prime}(u(t))=J^{*} T(-\lambda t)^{*} H^{\prime}(w(t))=T(-\lambda t)^{*} J^{*} H^{\prime}(w(t))$.

Thus we see that $u(t)=T(\lambda t) w(t)$ is a solution (2.1) if and only if

$$
\frac{d}{d t} \Im w=J^{*} H^{\prime}(w)+\lambda A^{*} \Im w .
$$

Recalling that $B=J^{*} A^{*} \mathfrak{I}$ and that $\left(J^{*}\right)^{2}=-I$ we see that

$$
J^{*} H^{\prime}(w)+\lambda A^{*} \Im w=J^{*}\left\{H^{\prime}(w)-\lambda B w\right\}=J^{*} D_{u} G_{\lambda}(w)
$$

and (IVP) is equivalent to the new Hamiltonian system

$$
\begin{aligned}
\frac{d}{d t} \Im w & =J^{*} D_{u} G_{\lambda}(w) \\
w(0) & =u_{0}
\end{aligned}
$$

where, as before, $G_{\lambda}(w)=H(w)-\lambda Q(w)$ with $Q(w)=\frac{1}{2}\langle B w, w\rangle$.

Since $D_{u} G_{\lambda}\left(\xi_{\lambda}\right)=0, w_{\lambda}(t)=\xi_{\lambda}$ is a stationary solution of (NHS). Thus the change of variable reduces the standing wave $u(t)=T(\lambda t) \xi_{\lambda}$ for (2.1) to the stationary solution $w(t)=\xi_{\lambda}$ for (NHS). Furthermore, (GWP 
at $\left.\xi_{\lambda}\right)$ ) implies that (NHS) is globally well-posed for initial conditions near $\xi_{\lambda}$ and that $H, Q$ and $G_{\lambda}$ are conserved quantities for (NHS). The orbit $\Theta\left(\xi_{\lambda}\right)$ consists of stationary solutions of (NHS).

Although we do not use (NHS) directly in what follows we hope that this reduction clarifies the role and origin of the augmented Hamiltonian $G_{\lambda}$. Indeed, the same change of variable reduces the linearization of (IVP) at $u(t)=T(\lambda t) \xi_{\lambda}$ to an autonomous form just as in the Floquet theory of linear periodic systems. The linearization of (IVP) is

$$
\frac{d}{d t} \Im z=J^{*} D_{u u}^{2} H\left(T(\lambda t) \xi_{\lambda}\right) z
$$

where $D_{u u}^{2} H\left(T(\lambda t) \xi_{\lambda}\right) z=T(-\lambda t)^{*} D_{u u}^{2} H\left(\xi_{\lambda}\right) T(-\lambda t) z$.

Setting $w(t)=T(-\lambda t) z(t)$ as above, we find that

$$
\frac{d}{d t} \mathfrak{I} z=T(-\lambda t)^{*}\left\{-\lambda A^{*} \mathfrak{I} w(t)+\frac{d}{d t} \mathfrak{I} w(t)\right.
$$

and hence that (LIVP) is equivalent to

$$
-\lambda A^{*} \Im w+\frac{d}{d t} \Im w=J^{*} D_{u u}^{2}\left(\xi_{\lambda}\right) w
$$

which can be written as

$$
\frac{d}{d t} \Im w=J^{*}\left\{D_{u u}^{2} H\left(\xi_{\lambda}\right)-\lambda B\right\} w .
$$

Note that $M=D_{u u}^{2} H\left(\xi_{\lambda}\right)-\lambda B=D_{u u}^{2} G_{\lambda}\left(\xi_{\lambda}\right) \in B\left(X, X^{*}\right)$ and that $M$ is symmetric:

$$
\langle M \xi, \eta\rangle=\langle M \eta, \xi\rangle \text { for all } \xi, \eta \in X,
$$

and that (LIVP) has been reduced to the linear autonomous Hamiltonian system

$$
\frac{d}{d t} \Im w=J^{*} M w
$$

similar to (2.5).

Remark. In the case where $(H,(\cdot \cdot),\|\cdot\|)=\left(X,\langle\cdot, \cdot\rangle_{X},\|\cdot\|_{X}\right)$, the linear system (4.1) reduces to

$$
\frac{d}{d t} w=J^{T} M w=-J M w
$$

and one might hope to establish the stability of the stationary solution $w(t)=\xi_{\lambda}$ of (NHS) by showing that $\sigma(J M) \subset\{z \in \mathbb{C}: \Re z>0\}$, where 
$\sigma(J M)$ denotes the spectrum of $J M$ on the complexification $X_{\mathbb{C}}$ of $X$ and $\Re z$ is the real part of $z$. However, for any $z \in \mathbb{C}$ we have that

$$
\begin{aligned}
& J M-z I: X_{\mathbb{C}} \rightarrow X_{\mathbb{C}} \text { is an isomorphism } \\
\Leftrightarrow & M-z J^{-1}: X_{\mathbb{C}} \rightarrow X_{\mathbb{C}} \text { is an isomorphism } \\
\Leftrightarrow & M+z J: X_{\mathbb{C}} \rightarrow X_{\mathbb{C}} \text { is an isomorphism } \\
\Leftrightarrow & M^{T}+\bar{z} J^{T}: X_{\mathbb{C}} \rightarrow X_{\mathbb{C}} \text { is an isomorphism } \\
\Leftrightarrow & M-\bar{z} J: X_{\mathbb{C}} \rightarrow X_{\mathbb{C}} \text { is an isomorphism } \\
\Leftrightarrow & J M-\bar{z} J^{2}: X_{\mathbb{C}} \rightarrow X_{\mathbb{C}} \text { is an isomorphism } \\
\Leftrightarrow & J M+\bar{z} I: X_{\mathbb{C}} \rightarrow X_{\mathbb{C}} \text { is an isomorphism, }
\end{aligned}
$$

showing that $z \in \sigma(J M) \Leftrightarrow-\bar{z} \in \sigma(J M)$. Hence we can never have $\sigma(J M) \subset\{z \in \mathbb{C}: \Re z>0\}$. In fact, since $J M$ is real, its spectrum is also symmetric with respect to the real axis. Combining these properties we see that $\sigma(J M)$ is symmetric with respect to both the real and imaginary axes. Thus it is only in situations where $\sigma(J M) \subset i \mathbb{R}$ that we can hope the establish even the stability of the standing wave $u(t)=T(\lambda t) \xi_{\lambda}$.

\section{2. $\Gamma$-invariant Lyapunov functions}

We suppose that, in addition to $(\mathrm{H})$, the condition (LWP) is also satisfied. Recall that, for $\xi \in X$ and $\rho>0$,

$$
\Theta(\xi)_{\rho}=\{v \in X: d(v, \Theta(\xi))<\rho\}
$$

where $d(v, \Theta(\xi))=\inf \left\{\|v-T(\theta) \xi\|_{X}: \theta \in \mathbb{R}\right\}$ and that $\Theta(\xi)_{\rho}$ is $\Gamma$-invariant in the sense that

$$
T(\theta) \Theta(\xi)_{\rho}=\Theta(\xi)_{\rho} \text { for all } \theta \in \mathbb{R} .
$$

A $\Gamma$-invariant Lyapunov function for the orbit $\Theta(\xi)$ is a function $V$ : $\Theta(\xi)_{\rho} \rightarrow \mathbb{R}$ having the following properties.

(a) There exists $\rho>0$ such that $V \in C^{2}\left(\Theta(\xi)_{\rho}\right)$ and

$$
V(\eta)=0 \text { and } V^{\prime}(\eta)=0 \text { for all } \eta \in \Theta(\xi) .
$$

(b) There exists $c>0$ such that

$$
V(\eta) \geq c d(\eta, \Theta(\xi))^{2} \text { for all } \eta \in \Theta(\xi)_{\rho} .
$$

(c) $\left\langle V^{\prime}(\eta), A \eta\right\rangle=0$ for all $\eta \in \Theta(\xi)_{\rho}$.

(d) $V(u(t))=V\left(u_{0}\right)$ for all $t \in\left[0, b_{+}\left(u_{0}\right)\right)$ and all $u_{0} \in \Theta\left(\xi_{\lambda}\right)_{\rho}$ where $u$ is the maximal solution of (IVP) with initial condition $u_{0}$. 
Remark 1. We have seen that

$$
T(\theta) \Theta\left(\xi_{\lambda}\right)_{\rho}=\Theta\left(\xi_{\lambda}\right)_{\rho} \text { for all } \theta \in \mathbb{R}
$$

and the condition (c) implies that

$$
V(T(\theta) \eta)=V(\eta) \text { for all } \eta \in \Theta\left(\xi_{\lambda}\right)_{\rho},
$$

showing that $V$ is $\Gamma$-invariant.

Proposition 4.1. Suppose that (3.1) and (GWP at $\xi_{\lambda}$ ) are satisfied and that there exists a $\Gamma$-invariant Lyapunov function, $V: \Theta(\xi)_{\rho} \rightarrow \mathbb{R}$, for the orbit $\Theta\left(\xi_{\lambda}\right)$. Then the standing wave $u_{\lambda}(t)=T(\lambda t) \xi_{\lambda}$ is orbitally stable

Proof. Fix $\varepsilon>0$. By the continuity of $V$ at $\xi_{\lambda}$, there exists $\delta \in(0, \rho)$ such that $V(\eta)=V(\eta)-V\left(\xi_{\lambda}\right)<c \min \left\{(\rho / 2)^{2}, \varepsilon^{2}\right\}$ for all $\eta \in B\left(\xi_{\lambda}, \delta\right)$. Hence $V(\eta)<c \min \left\{(\rho / 2)^{2}, \varepsilon^{2}\right\}$ for all $\eta \in \Theta\left(\xi_{\lambda}\right)_{\delta}$ by the $\Gamma$-invariance of $V$. By (GWP at $\xi_{\lambda}$ ) we can choose $\delta>0$ so that $b_{+}\left(u_{0}\right)=\infty$ for all $u_{0} \in B\left(\xi_{\lambda}, \delta\right)$. Let $u$ denote the solution of (IVP) with initial condition $u_{0}$ and set

$$
W=\left\{\tau>0: u(t) \in \Theta\left(\xi_{\lambda}\right)_{\rho} \text { for all } t \in[0, \tau)\right\} .
$$

Since $u \in C([0, \infty), X)$ and $0<\delta<\rho$, the set $W$ is a non-empty interval and $\inf W=0$. Let $\tau^{*}=\sup W$. If $\tau^{*}<\infty$, then for all $t<\tau^{*}, u(t) \in \Theta\left(\xi_{\lambda}\right)_{\rho}$ and so, by (b),

$$
c d\left(u(t), \Theta\left(\xi_{\lambda}\right)\right)^{2} \leq V(u(t))=V\left(u_{0}\right)<c(\rho / 2)^{2} .
$$

Thus $d\left(u(t), \Theta\left(\xi_{\lambda}\right)\right)<\rho / 2$ for all $t<\tau^{*}$ and, by continuity of $u$, $d\left(u\left(\tau^{*}\right), \Theta\left(\xi_{\lambda}\right)\right) \leq \rho / 2$. The continuity of $u$ now implies that $\tau^{*} \neq \sup W$, a contradiction. Hence $W=[0, \infty)$ and so for all $t \geq 0$, (b) now yields

$$
c d\left(u(t), \Theta\left(\xi_{\lambda}\right)\right)^{2} \leq V(u(t))=V\left(u_{0}\right)<c \varepsilon^{2} .
$$

Thus $d\left(u(t), \Theta\left(\xi_{\lambda}\right)\right)<\varepsilon$ for all $t \geq 0$, proving orbital stability.

The existence of a Lyapunov function means that (GWP at $\xi$ ) can be deduced from (LWP) provided that the following weaker version of the compactness condition is satisfied.

$(\mathrm{BCC})_{c, d} H^{\prime}: X \rightarrow X^{*}$ is bounded on bounded subsets of $X$ and there exists $\varepsilon>0$ such that, for any $h \in(c-\varepsilon, c+\varepsilon)$ and $q \in(d-\varepsilon, d+\varepsilon)$, any closed bounded subset of

$$
\{v \in X: H(v)=h\} \cap\{v \in X: Q(v)=q\}
$$

is a compact subset of $X$.

Remark. If $\operatorname{dim} X<\infty$ then $(\mathrm{BCC})_{c, d}$ holds for all $c, d \in \mathbb{R}$. 
Proposition 4.2. Suppose that (3.1) and (LWP) are satisfied and that there exists a $\Gamma$-invariant Lyapunov function for the orbit $\Theta\left(\xi_{\lambda}\right)$. If $(\mathrm{BCC})_{H\left(\xi_{\lambda}\right), Q\left(\xi_{\lambda}\right)}$ is satisfied, then (GWP at $\xi_{\lambda}$ ) holds and the standing wave $u_{\lambda}(t)=T(\lambda t) \xi_{\lambda}$ is orbitally stable.

Proof. By Proposition 4.1 it is enough to show that (GWP at $\xi_{\lambda}$ ) holds. Returning to the proof of Proposition 4.1, we redefine the set $W$ as

$$
W=\left\{\tau \in\left(0, b_{+}\left(u_{0}\right)\right): u(t) \in \Theta\left(\xi_{\lambda}\right)_{\rho} \text { for all } t \in[0, \tau)\right\} .
$$

The same argument now shows that $\tau^{*}=\sup W=b_{+}\left(u_{0}\right)$ for all $u_{0} \in$ $B\left(\xi_{\lambda}, \delta\right)$ and hence that $d\left(u(t), \Theta\left(\xi_{\lambda}\right)\right)<\varepsilon$ for all $t \in\left[0, b_{+}\left(u_{0}\right)\right)$. This implies that $\left\{\|u(t)\|_{X}: t \in\left[0, b_{+}\left(u_{0}\right)\right)\right\}$ is bounded and we can now repeat the proof of Proposition 2.2 with $(\mathrm{CC})_{H\left(\xi_{\lambda}\right), Q\left(\xi_{\lambda}\right)}$ replaced by $(\mathrm{BCC})_{H\left(\xi_{\lambda}\right), Q\left(\xi_{\lambda}\right)}$ to conclude that (GWP at $\xi_{\lambda}$ ) holds.

\subsection{Existence of a $\Gamma$-invariant Lyapunov function}

Given a pair $\left(\lambda, \xi_{\lambda}\right)$ satisfying (3.1), we define a function $V: X \rightarrow \mathbb{R}$ as follows. Let

$$
g=G_{\lambda}\left(\xi_{\lambda}\right) \text { and } q=Q\left(\xi_{\lambda}\right)
$$

and set

$$
V(\eta)=G_{\lambda}(\eta)-g+K\{Q(\eta)-q\}^{2} \text { for } \eta \in X .
$$

We shall show that for $K$ large enough $V$ is a $\Gamma$-invariant Lyapunov function for $\Theta\left(\xi_{\lambda}\right)$ provided that (SC) holds. For this we need the following lemma. Recall that $S_{\lambda}=R^{-1} D_{v v}^{2} G_{\lambda}\left(\xi_{\lambda}\right) \in B(X, X)$ is self-adjoint.

Lemma 4.3. Suppose that (SC) is satisfied. There exist $\delta>0$ and $K>0$ such that

$$
\begin{aligned}
\left\langle S_{\lambda} v, v\right\rangle_{X}+2 K\left\langle R^{-1} B \xi_{\lambda}, v\right\rangle_{X}^{2} & \geq \delta\|v\|_{X}^{2} \text { for all } v \in\left\{A \xi_{\lambda}\right\}^{\perp}, \\
\text { where }\left\{A \xi_{\lambda}\right\}^{\perp} & =\left\{v \in X:\left\langle v, A \xi_{\lambda}\right\rangle_{X}=0\right\} .
\end{aligned}
$$

Proof. We have already observed that $\left\langle R^{-1} B \xi_{\lambda}, A \xi_{\lambda}\right\rangle_{X}=0$. Hence for any $v \in\left\{A \xi_{\lambda}\right\}^{\perp}$, we have

$$
v=\alpha w+z
$$

where $w=R^{-1} B \xi_{\lambda} /\left\|R^{-1} B \xi_{\lambda}\right\|_{X}, \alpha=\langle v, w\rangle_{X}$ and $z \in\left\{A \xi_{\lambda}, R^{-1} B \xi_{\lambda}\right\}^{\perp}$.

Therefore

$$
\begin{aligned}
\left\langle S_{\lambda} v, v\right\rangle_{X} & =\alpha^{2}\left\langle S_{\lambda} w, w\right\rangle_{X}+2 \alpha\left\langle S_{\lambda} w, z\right\rangle_{X}+\left\langle S_{\lambda} z, z\right\rangle_{X} \\
& \geq \alpha^{2}\left\langle S_{\lambda} w, w\right\rangle_{X}+2 \alpha\left\langle S_{\lambda} w, z\right\rangle_{X}+\delta\|z\|_{X}^{2} \text { by (SC) } \\
& \geq \alpha^{2}\left\langle S_{\lambda} w, w\right\rangle_{X}-\left\{\frac{\delta}{2}\|z\|_{X}^{2}+\alpha^{2} \frac{2}{\delta}\left\|S_{\lambda} w\right\|_{X}^{2}\right\}+\delta\|z\|_{X}^{2}
\end{aligned}
$$


by Young's inequality. Setting $\beta=\left\|R^{-1} B \xi_{\lambda}\right\|_{X}$,

$$
\left\langle R^{-1} B \xi_{\lambda}, v\right\rangle_{X}=\langle\beta w, v\rangle_{X}=\alpha \beta
$$

and so

$$
\begin{aligned}
& \left\langle S_{\lambda} v, v\right\rangle_{X}+2 K\left\langle R^{-1} B \xi_{\lambda}, v\right\rangle_{X}^{2} \\
& \geq \alpha^{2}\left\langle S_{\lambda} w, w\right\rangle_{X}-\left\{\frac{\delta}{2}\|z\|_{X}^{2}+\alpha^{2} \frac{2}{\delta}\left\|S_{\lambda} w\right\|_{X}^{2}\right\}+\delta\|z\|_{X}^{2}+2 K \alpha^{2} \beta^{2} \\
& =\alpha^{2}\left\{\left\langle S_{\lambda} w, w\right\rangle_{X}-\frac{2}{\delta}\left\|S_{\lambda} w\right\|_{X}^{2}+2 K \beta^{2}\right\}+\frac{\delta}{2}\|z\|_{X}^{2} \\
& \geq \frac{\delta}{2}\left\{\alpha^{2}+\|z\|_{X}^{2}\right\}=\frac{\delta}{2}\|v\|_{X}^{2}
\end{aligned}
$$

provided that we choose $K>0$ such that

$$
\left\langle S_{\lambda} w, w\right\rangle_{X}-\frac{2}{\delta}\left\|S_{\lambda} w\right\|_{X}^{2}+2 K \beta^{2} \geq \frac{\delta}{2} .
$$

Theorem 4.4. Suppose that (3.1) and (SC) are satisfied. There exist $K>0$ and $\rho>0$ such that (LF) defines a $\Gamma$-invariant Lyapunov function for $\Theta\left(\xi_{\lambda}\right)$ on $\Theta\left(\xi_{\lambda}\right)_{\rho}$.

Proof. First we observe that the function defined by (LF) has the properties (c) and (d) because $G_{\lambda}$ and $Q$ have these properties. In particular, $V$ is $\Gamma$ invariant. Furthermore $V \in C^{2}(X)$ and, for all $\eta, v \in X$,

$$
\begin{aligned}
\left\langle V^{\prime}(\eta), v\right\rangle= & \left\langle D_{u} G_{\lambda}(\eta), v\right\rangle+2 K\{Q(\eta)-q\}\left\langle Q^{\prime}(\eta), v\right\rangle \\
\left\langle V^{\prime \prime}(\eta) v, v\right\rangle= & \left\langle D_{u u}^{2} G_{\lambda}(\eta) v, v\right\rangle+2 K\{Q(\eta)-q\}\left\langle Q^{\prime \prime}(\eta) v, v\right\rangle \\
& +2 K\left\langle Q^{\prime}(\eta), v\right\rangle^{2} .
\end{aligned}
$$

Thus, for all $v \in X$,

$$
\begin{aligned}
\left\langle V^{\prime}\left(\xi_{\lambda}\right), v\right\rangle & =0, \\
\left\langle V^{\prime \prime}\left(\xi_{\lambda}\right) v, v\right\rangle & =\left\langle D_{u u}^{2} G_{\lambda}\left(\xi_{\lambda}\right) v, v\right\rangle+2 K\left\langle Q^{\prime}\left(\xi_{\lambda}\right), v\right\rangle^{2} \\
& =\left\langle S_{\lambda} v, v\right\rangle_{X}+2 K\left\langle R^{-1} B \xi_{\lambda}, v\right\rangle_{X}^{2} .
\end{aligned}
$$

Hence $V\left(\xi_{\lambda}\right)=0$ and $V^{\prime}\left(\xi_{\lambda}\right)=0$. It only remains to check property (b).

Since $A \xi_{\lambda} \neq 0$, there is a constant $R\left(\xi_{\lambda}\right)$ such that Lemma 2.1 holds.

By Lemma 4.3, there exists $\delta>0$ such that

$$
\left\langle V^{\prime \prime}\left(\xi_{\lambda}\right) v, v\right\rangle \geq \delta\|v\|_{X}^{2} \text { for all } v \in\left\{A \xi_{\lambda}\right\}^{\perp} .
$$


Since $V \in C^{2}(X)$, there exists $\rho \in\left(0, \frac{1}{2} R\left(\xi_{\lambda}\right)\right)$ such that

$$
V(\eta)=V\left(\xi_{\lambda}\right)+\left\langle V^{\prime}\left(\xi_{\lambda}\right), \eta-\xi_{\lambda}\right\rangle+\frac{1}{2}\left\langle V^{\prime \prime}\left(\xi_{\lambda}\right)\left[\eta-\xi_{\lambda}\right], \eta-\xi_{\lambda}\right\rangle+r(\eta)
$$

where $|r(\eta)| \leq \frac{1}{4} \delta\left\|\eta-\xi_{\lambda}\right\|_{X}^{2}$ for all $\eta \in B\left(\xi_{\lambda}, \rho\right)$.

But $V\left(\xi_{\lambda}\right)=0$ and $\left\langle V^{\prime}\left(\xi_{\lambda}\right), \eta-\xi_{\lambda}\right\rangle=0$ for all $\eta \in X$. Hence,

$$
\begin{aligned}
V(\eta) & =\frac{1}{2}\left\langle V^{\prime \prime}\left(\xi_{\lambda}\right)\left[\eta-\xi_{\lambda}\right], \eta-\xi_{\lambda}\right\rangle+r(\eta) \\
& \geq \frac{1}{2} \delta\left\|\eta-\xi_{\lambda}\right\|_{X}^{2}-\frac{1}{4} \delta\left\|\eta-\xi_{\lambda}\right\|_{X}^{2}=\frac{1}{4} \delta\left\|\eta-\xi_{\lambda}\right\|_{X}^{2} \\
& \geq \frac{1}{4} \delta d\left(\eta, \Theta\left(\xi_{\lambda}\right)\right)^{2},
\end{aligned}
$$

provided that $\left\|\eta-\xi_{\lambda}\right\|_{X}<\rho$ and $\left\langle\eta-\xi_{\lambda}, A \xi_{\lambda}\right\rangle_{X}=0$.

Now consider any $v \in \Theta\left(\xi_{\lambda}\right)_{\rho}$. By Lemma 2.1 there exists $\theta_{1} \in \mathbb{R}$ such that $\left\|v-T\left(\theta_{1}\right) \xi_{\lambda}\right\|_{X}<\rho$ and $\left\langle v-T\left(\theta_{1}\right) \xi_{\lambda}, A T\left(\theta_{1}\right) \xi_{\lambda}\right\rangle_{X}=0$. Setting $\eta=T\left(-\theta_{1}\right) v$, this means that

$$
\left\|\eta-\xi_{\lambda}\right\|_{X}<\rho \text { and }\left\langle\eta-\xi_{\lambda}, A \xi_{\lambda}\right\rangle_{X}=0
$$

Hence by (4.3), $V(v)=V(\eta) \geq \frac{1}{4} \delta d\left(\eta, \Theta\left(\xi_{\lambda}\right)\right)^{2}=\frac{1}{4} \delta d\left(v, \Theta\left(\xi_{\lambda}\right)\right)^{2}$ for all $v \in \Theta\left(\xi_{\lambda}\right)_{\rho}$, showing that $V$ has the property (b).

\subsection{Stability of the stationary solution $u \equiv 0$}

When $H^{\prime}(0)=0$, we have that $u(t) \equiv 0$ is a solution of (2.1). Its orbit is a single point, $\Theta(0)=\{0\}$, and orbital stability is just stability in the usual sense. Under appropriate conditions this can also be established by the construction of a Lyapunov function.

Theorem 4.5. Suppose that $H^{\prime}(0)=0$ and that (GWP at 0 ) is satisfied. If there exist $\lambda \in \mathbb{R}$ and $\delta>0$ such that

$$
\left\langle D_{u u}^{2} G_{\lambda}(0) v, v\right\rangle=\left\langle H^{\prime \prime}(0) v, v\right\rangle-2 \lambda Q(v) \geq \delta\|v\|_{X}^{2} \text { for all } v \in X,
$$

then there exists $\rho>0$ such that $V(\eta)=G_{\lambda}(\eta)-G_{\lambda}(0)$ is a $\Gamma$-invariant Lyapunov function for $\Theta(0)=\{0\}$ on $\Theta(0)_{\rho}=B(0, \rho)$ and, consequently, $u \equiv 0$ is a stable solution of (IVP).

Proof. A simplified version of Proposition 4.1 shows that the existence of a Lyapunov function implies the stability of $u \equiv 0$. In checking that $G_{\lambda}-$ $G_{\lambda}(0)$ is a Lyapunov function, the properties (a), (c) and (d) are easily seen 
to hold since $G_{\lambda}(0)=0$ and $D_{u} G_{\lambda}(0)=0$. Also $G_{\lambda} \in C^{2}(X, \mathbb{R})$, so there exists $\rho>0$ such that

$$
G_{\lambda}(\eta)=\frac{1}{2}\left\langle D_{u u}^{2} G_{\lambda}(0) \eta, \eta\right\rangle+r(\eta)
$$

where $|r(\eta)| \leq \frac{1}{4} \delta\|\eta\|_{X}^{2}$ for all $\eta \in B(0, \rho)$. Thus $G_{\lambda}(\eta) \geq \frac{1}{4} \delta\|\eta\|_{X}^{2}$ for all $\eta \in B(0, \rho)$, proving property (b).

\section{A branch of standing waves}

Solutions of (3.1) are often embedded in smooth families parameterized by $\lambda$. Provided that the Morse index of $D_{v v}^{2} G_{\lambda}\left(\xi_{\lambda}\right)$ is 1 along this branch $\left(\lambda, \xi_{\lambda}\right)$, the stability of the corresponding standing waves is determined by the monotonicity of $Q\left(\xi_{\lambda}\right)$ with respect to $\lambda$. This is sometimes referred to as the Vahkitov-Kolokolov criterion and it was thoroughly investigated by Grillakis, Shatah and Strauss, [14]. To be more precise we formulate the following conditions (A2) and (A3) which correspond to Assumptions 2 and 3 in [14].

(A2) (Existence of a branch of standing waves) There exist an open interval $(a, b) \subset \mathbb{R} \backslash\{0\}$ and a function $\xi \in C^{1}((a, b), X)$ such that $A \xi_{\lambda} \neq 0$ and $H^{\prime}\left(\xi_{\lambda}\right)=\lambda B\left(\xi_{\lambda}\right)$ for all $\lambda \in(a, b)$ where $\xi_{\lambda}=\xi(\lambda)$.

Remark. If $H^{\prime}(0)=0$, it may be that there is a branch of solutions $\left(\lambda, u_{\lambda}\right)$ such that $A \xi_{\lambda} \neq 0$ but $\left\|u_{\lambda}\right\|_{X} \rightarrow 0$ as $\lambda \rightarrow a$. In this case, $\lambda=a$ is a bifurcation point for the equation $H^{\prime}(\xi)=\lambda B \xi$ and the standing waves $T(\lambda t) \xi_{\lambda}$ converge to the stationary solution $u(t) \equiv 0$ of (2.1). Conversely, it may be possible to use bifurcation theory to establish the existence of such a branch of solutions of $H^{\prime}(\xi)=\lambda B \xi$, as in the classical Lyapunov Centre Theorem in finite dimensions. See [1], for example. In Section 8, we present several bifurcation results of this kind in the context of the nonlinear Schrödinger equation. Of course, the bifurcation may occur at $\lambda=b$ rather than at $\lambda=a$.

Recalling that $H^{\prime \prime}(v)-\lambda B=D_{u u}^{2} G_{\lambda}(v)$ where $G_{\lambda}=H-\lambda Q$, we next we consider the spectrum of the Hessian of the augmented Hamiltonian. By (3.2),

$$
S_{\lambda}=R^{-1} D_{u u}^{2} G_{\lambda}\left(\xi_{\lambda}\right)=R^{-1}\left\{H^{\prime \prime}\left(\xi_{\lambda}\right)-\lambda B\right\} \text { for } \lambda \in(a, b) .
$$

Using (A2), $S \in C((a, b), B(X, X))$ and, for all $v, w \in X$,

$$
\left\langle S_{\lambda} v, w\right\rangle_{X}=\left\langle\left\{H^{\prime \prime}\left(\xi_{\lambda}\right)-\lambda B\right\} v, w\right\rangle=\left\langle\left\{H^{\prime \prime}\left(\xi_{\lambda}\right)-\lambda B\right\} w, v\right\rangle=\left\langle v, S_{\lambda} w\right\rangle_{X}
$$


showing that $S_{\lambda}$ is self-adjoint on $\left(X,\langle\cdot, \cdot\rangle_{X}\right)$. Let $\sigma\left(S_{\lambda}\right)$ and $\sigma_{e}\left(S_{\lambda}\right)$ denote the spectrum and essential spectrum of $S_{\lambda}$.

(A3) (Spectrum of the Hessian) For all $\lambda \in(a, b)$,

(i) $S_{\lambda}$ has exactly one eigenvalue in $(-\infty, 0)$ and it is simple.

(ii) $\operatorname{ker} S_{\lambda}=\operatorname{span}\left\{A \xi_{\lambda}\right\}$.

(iii) There exists $\varepsilon_{\lambda}>0$ such that $\left(0, \varepsilon_{\lambda}\right) \cap \sigma\left(S_{\lambda}\right)=\emptyset$.

Remarks. Recalling (2.4), we see that (A2) implies that $\xi_{\lambda}$ is a degenerate critical point of $G_{\lambda}$. The assumption (A3)(ii) means that ker $D_{u u}^{2} G_{\lambda}\left(\xi_{\lambda}\right)=$ $\operatorname{span}\left\{A \xi_{\lambda}\right\}$.

Let $M\left(S_{\lambda}\right)$ denote the Morse index of $S_{\lambda}$ (i.e. the maximal dimension of subspaces on which $S_{\lambda}$ is negative definite). The assumption (A3)(i) means that $M\left(S_{\lambda}\right)=1$. Thus $\xi_{\lambda}$ is a saddle point of $G_{\lambda}$. Given parts (i) and (ii), part (iii) of (A3) asserts that inf $\sigma_{e}\left(S_{\lambda}\right)>0$ for all $\lambda \in(a, b)$. In particular, the Hessian $D_{u u}^{2} G_{\lambda}\left(\xi_{\lambda}\right): X \rightarrow X^{*}$ is a Fredholm operator of index zero.

As we now show, if $\left(\mu, \xi_{\mu}\right)$ satisfies (3.1) and if $S_{\mu}$ has the properties (i), (ii) and (iii), then the conditions (A2) and (A3) are satisfied on an open interval $(a, b)$ containing $\mu$. To this end we introduce the following hypothesis.

(B) There exist $\mu \in \mathbb{R} \backslash\{0\}$ and $\xi_{\mu} \in X$ such that

$$
\begin{aligned}
A \xi_{\mu} & \neq 0, D_{u} G_{\mu}\left(\xi_{\mu}\right)=0, \\
\inf \sigma_{e}\left(S_{\mu}\right) & >0, M\left(S_{\mu}\right)=1 \text { and } \operatorname{ker} S_{\mu}=\operatorname{span}\left\{A \xi_{\mu}\right\} .
\end{aligned}
$$

where $S_{\mu}=R^{-1} D_{u u}^{2} G_{\mu}\left(\xi_{\mu}\right)$.

Theorem 5.1. If (B) holds there exists an open interval $(a, b)$ containing $\mu$ on which (A2) and (A3) are satisfied.

Remark. According to (A3)(i), there exist $\gamma_{\lambda}>0$ and $\eta_{\lambda} \in X \backslash\{0\}$ such that

$$
\sigma\left(S_{\lambda}\right) \cap(-\infty, 0)=\left\{-\gamma_{\lambda}\right\} \text { and } \operatorname{ker}\left\{S_{\lambda}+\gamma_{\lambda} I\right\}=\operatorname{span}\left\{\eta_{\lambda}\right\} .
$$

Since ker $S_{\lambda}=\left\{A \xi_{\lambda}\right\}$ and $S_{\lambda}: X \rightarrow X$ is self-adjoint, $\left\langle A \xi_{\lambda}, \eta_{\lambda}\right\rangle_{X}=0$. Also (A3)(ii) and (iii) imply that there exists $\delta>0$ such that

$$
\left\langle S_{\lambda} z, z\right\rangle_{X} \geq \delta\|z\|_{X}^{2} \text { for all } z \in\left\{A \xi_{\lambda}, \eta_{\lambda}\right\}^{\perp} .
$$

This means that $S_{\lambda}$ is positive definite on a subspace of codimension 2, but this alone does not guarantee that (SC) is satisfied since (SC) requires 
positive definiteness on $\left\{A \xi_{\lambda}, R^{-1} B \xi_{\lambda}\right\}^{\perp}$. We return to this issue after the proof of Theorem 5.1.

Proof. We use the implicit function theorem. Let

$$
\begin{aligned}
\varphi & =\frac{A \xi_{\mu}}{\left\|A \xi_{\mu}\right\|_{X}}, Y=\varphi^{\perp}=\left\{v \in X:\langle v, \varphi\rangle_{X}=0\right\} \text { and } \\
g(\lambda, v) & =R^{-1} D_{u} G_{\lambda}(v) \text { for }(\lambda, v) \in \mathbb{R} \times X .
\end{aligned}
$$

Then define $F: \mathbb{R} \times X \rightarrow \mathbb{R} \times Y$ by

$$
F(\lambda, v)=\left(\left\langle v, A \xi_{\mu}\right\rangle_{X}, g(\lambda, v)-\langle g(\lambda, v), \varphi\rangle_{X} \varphi\right) .
$$

Then $F \in C^{1}(\mathbb{R} \times X, \mathbb{R} \times Y)$ with $F\left(\mu, \xi_{\mu}\right)=(0,0)$ and

$$
\begin{aligned}
& \left.D_{u} F\left(\mu, \xi_{\mu}\right) w=\left(\left\langle w, A \xi_{\mu}\right\rangle_{X}, S_{\mu} w-\left\langle S_{\mu} w, \varphi\right\rangle_{X} \varphi\right)\right) \\
& =\left(\left\langle w, A \xi_{\mu}\right\rangle_{X}, S_{\mu} w\right) \text { since }\left\langle S_{\mu} w, \varphi\right\rangle_{X}=\left\langle w, S_{\mu} \varphi\right\rangle_{X}=0 .
\end{aligned}
$$

The condition (B) implies that $D_{u} F\left(\mu, \xi_{\mu}\right): X \rightarrow \mathbb{R} \times Y$ is an isomorphism. Hence there exist an open interval $(a, b)$ containing $\mu$ and a function $\xi \in$ $C^{1}((a, b), X)$ such that

$$
\xi(\mu)=\xi_{\mu} \text { and } F(\lambda, \xi(\lambda))=0 \text { for all } \lambda \in(a, b) .
$$

In particular,

$$
g(\lambda, \xi(\lambda))-\langle g(\lambda, \xi(\lambda)), \varphi\rangle_{X} \varphi=0
$$

But

$$
\begin{aligned}
\langle g(\lambda, \xi(\lambda)), A \xi(\lambda)\rangle_{X} & =\left\langle D_{u} G_{\lambda}(\xi(\lambda)), A \xi(\lambda)\right\rangle \\
& =\left\langle H^{\prime}(\xi(\lambda))-\lambda Q^{\prime}(\xi(\lambda)), A \xi(\lambda)\right\rangle=0
\end{aligned}
$$

for all $\lambda \in(a, b)$ by the $\Gamma$-invariance of $G_{\lambda}$. Hence

$$
\langle g(\lambda, \xi(\lambda)), \varphi\rangle_{X}\langle\varphi, A \xi(\lambda)\rangle_{X}=0 .
$$

But

$$
\langle\varphi, A \xi(\lambda)\rangle_{X} \rightarrow\langle\varphi, A \xi(\mu)\rangle_{X}=\|A \xi(\mu)\|_{X} \neq 0 \text { as } \lambda \rightarrow \mu .
$$

It follows that $\langle g(\lambda, \xi(\lambda)), \varphi\rangle_{X}=0$ for all $\lambda$ near $\mu$ and so, by reducing $(a, b)$ if necessary, we have that $g(\lambda, \xi(\lambda))=0$ for all $\lambda \in(a, b)$. Thus (A2) holds.

Since $S_{\lambda} \in B(X, X)$ is self-adjoint and $S_{\lambda} \rightarrow S_{\mu}$ as $\lambda \rightarrow \mu$, we may suppose that $\inf \sigma_{e}\left(S_{\lambda}\right) \geq \frac{1}{2} \inf \sigma_{e}\left(S_{\mu}\right)>0$ for all $\lambda \in(a, b)$. Since $M\left(S_{\mu}\right)=$ 1 and there exists $\varepsilon>0$ such that $\varepsilon \notin \sigma\left(S_{\mu}\right)$ and $M\left(S_{\mu}-\varepsilon I\right)=2$, we have that for $\lambda$ near $\mu, M\left(S_{\lambda}-\varepsilon I\right)=2$ and $M\left(S_{\lambda}\right) \geq 1$. But we know that $A \xi_{\lambda} \in \operatorname{ker} S_{\lambda}$ so we must have $M\left(S_{\lambda}\right)=1$ and $\operatorname{ker} S_{\lambda}=\operatorname{span}\left\{A \xi_{\lambda}\right\}$. Thus (A3) also holds. 


\subsection{The Vahkitov-Kolokolov criterion}

Under the hypotheses of Theorem 5.1, the following condition (VK) ensures that (SC) can be deduced from (5.1) and hence that there is orbital stability of the corresponding standing waves.

Proposition 5.2. Suppose that (B) holds and consider the branch of critical points $\lambda \mapsto \xi_{\lambda}$ given by Theorem 5.1. The condition (SC) is satisfied provided that

$$
\frac{d}{d \lambda} Q\left(\xi_{\lambda}\right)<0
$$

Consequently, if (GWP at $\left.\xi_{\lambda}\right)$, (B) and (VK) are satisfied, the standing wave $u_{\lambda}(t)=T(\lambda t) \xi_{\lambda}$ is orbitally stable.

Remark. The condition (VK) seems to have been introduced as a criterion for orbital stability by Vakhitov and Kolokolov (1973)[32], see also Zakharov and Shabat, [35]. A thorough examination of this condition was undertaken by Grillakis, Shatah and Strauss, [14]. Setting $d(\lambda)=G_{\lambda}\left(\xi_{\lambda}\right)=H\left(\xi_{\lambda}\right)-$ $\lambda Q\left(\xi_{\lambda}\right)$ and $\xi_{\lambda}^{\prime}=\frac{d}{d \lambda} \xi_{\lambda}$, we have

$$
\begin{aligned}
& d^{\prime}(\lambda)=\left\langle D_{u} G_{\lambda}\left(\xi_{\lambda}\right), \xi_{\lambda}^{\prime}\right\rangle-Q\left(\xi_{\lambda}\right)=-Q\left(\xi_{\lambda}\right), \\
& d^{\prime \prime}(\lambda)=-\frac{d}{d \lambda} Q\left(\xi_{\lambda}\right)
\end{aligned}
$$

and so the condition (VK) can be expressed as $d^{\prime \prime}(\lambda)>0$. See [14].

To show that (VK) implies (SC) we use the following lemma.

Lemma 5.3. Let $S: X \rightarrow X$ be any bounded self-adjoint operator such that

$$
\inf \sigma_{e}(S)>0, M(S)=1 \text { and } \operatorname{dim} \operatorname{ker} S=1 .
$$

Let $\chi$ be any element of $X$ such that $\langle S \chi, \chi\rangle_{X}<0$. Then there exists $\delta>0$ such that $\langle S z, z\rangle_{X} \geq \delta\|z\|_{X}^{2}$ for all $z \in\{\varphi, S \chi\}^{\perp}=\left\{z \in X:\langle z, \varphi\rangle_{X}=\right.$ $\left.\langle z, S \chi\rangle_{X}=0\right\}$ where $\operatorname{ker} S=\operatorname{span}\{\varphi\}$.

Proof. From the hypotheses, we have that there exist $\gamma>0$ and $\varepsilon>0$ such that, for some $\psi \in X \backslash\{0\}$,

$$
S \psi=-\gamma \psi \text { and } \sigma(S) \cap(-\infty, \varepsilon)=\{-\gamma, 0\} .
$$

Thus $\langle S v, v\rangle_{X} \geq \varepsilon\|v\|_{X}^{2}$ for all $v \in\{\varphi, \psi\}^{\perp}$ and we can suppose that $\|\varphi\|_{X}=\|\psi\|_{X}=1$ and $\langle\varphi, \psi\rangle_{X}=0$. Since $S$ is positive definite and symmetric on the $S$-invariant subspace $\{\varphi, \psi\}^{\perp}$, we have the Cauchy-Schwartz inequality:

$$
\left|\langle S v, w\rangle_{X}\right| \leq\langle S v, v\rangle_{X}^{1 / 2}\langle S w, w\rangle_{X}^{1 / 2} \text { for all } v, w \in\{\varphi, \psi\}^{\perp} .
$$


We can write the element $\chi$ as

$$
\chi=p \psi+q \varphi+v_{0}
$$

where $p=\langle\chi, \psi\rangle_{X}, q=\langle\chi, \varphi\rangle_{X}$ and $v_{0} \in\{\varphi, \psi\}^{\perp}$. Then

$$
\begin{aligned}
0 & >\langle S \chi, \chi\rangle_{X}=\left\langle-p \gamma \psi+S v_{0}, p \psi+q \varphi+v_{0}\right\rangle_{X} \\
& =-p^{2} \gamma+\left\langle v_{0}, p S \psi+S v_{0}\right\rangle_{X}=-p^{2} \gamma+\left\langle v_{0},-p \gamma \psi+S v_{0}\right\rangle_{X} \\
& =-p^{2} \gamma+\left\langle v_{0}, S v_{0}\right\rangle_{X}
\end{aligned}
$$

showing that

$$
\begin{aligned}
\left\langle S v_{0}, v_{0}\right\rangle_{X} & =p^{2} \gamma+\langle S \chi, \chi\rangle_{X} \text { and } \\
p^{2} \gamma & >\left\langle S v_{0}, v_{0}\right\rangle_{X} \geq \varepsilon\left\|v_{0}\right\|_{X}^{2} .
\end{aligned}
$$

In particular, $p \neq 0$.

Now consider any $z \in\{\varphi, S \chi\}^{\perp}$. We can also write $z$ as

$$
z=r \psi+s \varphi+v
$$

where $r=\langle z, \psi\rangle_{X}, s=\langle z, \varphi\rangle_{X}=0$ and $v \in\{\varphi, \psi\}^{\perp}$. Thus $z=r \psi+v$ and

$$
\begin{aligned}
\langle S z, z\rangle_{X} & =\langle-r \gamma \psi+S v, r \psi+v\rangle_{X}=-r^{2} \gamma+\langle S v, r \psi+v\rangle_{X} \\
& =-r^{2} \gamma+\langle S v, v\rangle_{X} \text { since }\langle S v, r \psi\rangle_{X}=r\langle v,-\gamma \psi\rangle_{X}=0 .
\end{aligned}
$$

Furthermore,

$$
\|z\|_{X}^{2}=r^{2}+\|v\|_{X}^{2}
$$

and

$$
0=\langle z, S \chi\rangle_{X}=\left\langle r \psi+v,-p \gamma \psi+S v_{0}\right\rangle_{X}=-r p \gamma+\left\langle v, S v_{0}\right\rangle_{X}
$$

Hence

$$
r=\frac{\left\langle v, S v_{0}\right\rangle_{X}}{p \gamma}
$$

and then 


$$
\begin{aligned}
\langle S z, z\rangle_{X} & =-r^{2} \gamma+\langle S v, v\rangle_{X}=-\frac{\left\langle v_{0}, S v\right\rangle_{X}^{2}}{p^{2} \gamma}+\langle S v, v\rangle_{X} \\
& \geq-\frac{\langle v, S v\rangle_{X}\left\langle v_{0}, S v_{0}\right\rangle_{X}}{p^{2} \gamma}+\langle S v, v\rangle_{X} \\
& =-\frac{\langle v, S v\rangle_{X}\left\{p^{2} \gamma+\langle S \chi, \chi\rangle_{X}\right\}}{p^{2} \gamma}+\langle S v, v\rangle_{X} \\
& =-\frac{\langle v, S v\rangle_{X}\langle S \chi, \chi\rangle_{X}}{p^{2} \gamma} \\
& \geq\left\{-\frac{\langle S \chi, \chi\rangle_{X}}{p^{2} \gamma}\right\} \varepsilon\|v\|_{X}^{2} \text { since }\langle S \chi, \chi\rangle_{X}<0 .
\end{aligned}
$$

But

$$
\begin{aligned}
\|z\|_{X}^{2} & =r^{2}+\|v\|_{X}^{2}=\left[\frac{\left\langle v, S v_{0}\right\rangle_{X}}{p \gamma}\right]^{2}+\|v\|_{X}^{2} \\
& \leq\left\{\frac{\left\|S v_{0}\right\|_{X}^{2}}{p^{2} \gamma^{2}}+1\right\}\|v\|_{X}^{2}
\end{aligned}
$$

so

$$
\begin{aligned}
\langle S z, z\rangle_{X} & \geq\left\{-\frac{\langle S \chi, \chi\rangle_{X}}{p^{2} \gamma}\right\} \varepsilon\|v\|_{X}^{2} \\
& \geq\left\{-\frac{\langle S \chi, \chi\rangle_{X}}{p^{2} \gamma}\right\} \varepsilon\left\{\frac{\left\|S v_{0}\right\|_{X}^{2}}{p^{2} \gamma^{2}}+1\right\}^{-1}\|z\|_{X}^{2} \\
& =\frac{\left\{-\langle S \chi, \chi\rangle_{X}\right.}{\| S \gamma \varepsilon}\|z\|_{X}^{2} .
\end{aligned}
$$

Finally, $S v_{0}=S\{\chi-p \psi-q \varphi\}=S \chi+p \gamma \psi$ and hence

$$
\begin{aligned}
\left\|S v_{0}\right\|_{X}^{2} & =\|S \chi\|_{X}^{2}+p^{2} \gamma^{2}+2\langle S \chi, p \gamma \psi\rangle_{X} \\
& =\|S \chi\|_{X}^{2}+p^{2} \gamma^{2}-2\left\langle\chi, p \gamma^{2} \psi\right\rangle_{X} \\
& =\|S \chi\|_{X}^{2}-p^{2} \gamma^{2} \text { since }\langle\chi, \psi\rangle_{X}=p .
\end{aligned}
$$

Therefore,

$$
\langle S z, z\rangle_{X} \geq \frac{\left\{-\langle S \chi, \chi\rangle_{X}\right\} \gamma \varepsilon}{\left\|S v_{0}\right\|_{X}^{2}+p^{2} \gamma^{2}}\|z\|_{X}^{2}=\frac{\left\{-\langle S \chi, \chi\rangle_{X}\right\} \gamma \varepsilon}{\|S \chi\|_{X}^{2}}\|z\|_{X}^{2} .
$$

Setting

$$
\delta=\frac{\left\{-\langle S \chi, \chi\rangle_{X}\right\} \gamma \varepsilon}{\|S \chi\|_{X}^{2}},
$$

we have $\langle S z, z\rangle_{X} \geq \delta\|z\|_{X}^{2}$ for all $z \in\{\varphi, S \chi\}^{\perp}$. 
Proof of Proposition 5.2. We claim that the hypotheses of Lemma 5.3 are satisfied by $S_{\lambda}$ with $\varphi=A \xi_{\lambda}$ and $\chi=\xi_{\lambda}^{\prime}$ where $\xi_{\lambda}^{\prime}=\frac{d}{d \lambda} \xi_{\lambda}$. Indeed, since $D_{v} G_{\lambda}\left(\xi_{\lambda}\right)=0$ for all $\lambda \in(a, b)$, we have that

$$
D_{v v}^{2} G_{\lambda}\left(\xi_{\lambda}\right) \xi_{\lambda}^{\prime}+D_{\lambda} D_{v} G_{\lambda}\left(\xi_{\lambda}\right)=0
$$

that is

$$
D_{v v}^{2} G_{\lambda}\left(\xi_{\lambda}\right) \xi_{\lambda}^{\prime}-Q^{\prime}\left(\xi_{\lambda}\right)=0
$$

Hence

$$
\left\langle S_{\lambda} \xi_{\lambda}^{\prime}, \xi_{\lambda}^{\prime}\right\rangle_{X}=\left\langle D_{v v}^{2} G_{\lambda}\left(\xi_{\lambda}\right) \xi_{\lambda}^{\prime}, \xi_{\lambda}^{\prime}\right\rangle=\left\langle Q^{\prime}\left(\xi_{\lambda}\right), \xi_{\lambda}^{\prime}\right\rangle=\frac{d}{d \lambda} Q\left(\xi_{\lambda}\right)<0 .
$$

as required for Lemma 5.3 which now implies that there exists $\delta>0$ such that $\left\langle S_{\lambda} z, z\right\rangle_{X} \geq \delta\|z\|_{X}^{2}$ for all $z \in\left\{A \xi_{\lambda}, S_{\lambda} \xi_{\lambda}^{\prime}\right\}^{\perp}$.

Finally we note that, by $(5.2)$,

$$
S_{\lambda} \xi_{\lambda}^{\prime}=R^{-1} D_{u u}^{2} G_{\lambda}\left(\xi_{\lambda}\right) \xi_{\lambda}^{\prime}=R^{-1} Q^{\prime}\left(\xi_{\lambda}\right)=R^{-1} B\left(\xi_{\lambda}\right)
$$

completing the proof.

When the Hessian of $G_{\mu}$ has the property (G), the condition (B) can be reformulated using the operator $L_{\mu}$ defined in Lemma 3.3. We begin by relating some properties of $L_{\mu}$ to those of $S_{\mu}$.

Lemma 5.4. Suppose that for some $\xi_{\mu} \in X$, the Hessian $D_{v v}^{2} G_{\mu}\left(\xi_{\mu}\right)$ has the property $(\mathrm{G})$ and that inf $\sigma_{e}\left(L_{\mu}\right)>0$. Then

$$
\inf \sigma_{e}\left(S_{\mu}\right)>0, M\left(S_{\mu}\right)=M\left(L_{\mu}\right) \text { and } \operatorname{ker} S_{\mu}=\operatorname{ker} L_{\mu} .
$$

Proof. Using the definition of $L_{\mu}$, it is easy to see that ker $S_{\mu}=\operatorname{ker} D_{v v}^{2} G_{\mu}\left(\xi_{\mu}\right)$ $\subset D\left(L_{\mu}\right)$ and hence that $\operatorname{ker} L_{\mu}=\operatorname{ker} S_{\mu}$.

Since $\inf \sigma_{e}\left(L_{\mu}\right)>0, M\left(L_{\mu}\right)<\infty$ and $d=\operatorname{dim} \operatorname{ker} L_{\mu}<\infty$. Also there exists $\Gamma>0$ such that $(0, \Gamma] \cap \sigma\left(L_{\mu}\right)=\emptyset$. Let $P \in B(H, H)$ denote the orthogonal projection onto the span of all the eigenvectors of $L_{\mu}$ corresponding the eigenvalues in $(-\infty, 0]$. and let $Q=I-P$. We have that $P(H) \subset D\left(L_{\mu}\right)$ and that $Q(u) \in D\left(L_{\mu}\right)$ if and only if $u \in D\left(L_{\mu}\right)$.Then $\operatorname{dim} P(H)=d+M\left(L_{\mu}\right)$ and $\left(L_{\mu} Q(z), Q(z)\right) \geq \Gamma\|Q(z)\|^{2}$ for all $z \in D\left(L_{\mu}\right)$. Thus, for any $z \in D\left(L_{\mu}\right)$,

$$
\begin{aligned}
\left\langle S_{\mu} z, z\right\rangle_{X} & =\left\langle D_{v v}^{2} G\left(\xi_{\mu}\right) z, z\right\rangle=\left(L_{\mu} z, z\right)=\left(Q L_{\mu} z, z\right)+\left(P L_{\mu} z, z\right) \\
& =\left(L_{\mu} Q z, Q z\right)+\left(P L_{\mu} z, z\right) \geq \Gamma\|Q z\|^{2}+\left(P L_{\mu} z, z\right) \\
& =\Gamma\|z\|^{2}-\Gamma\|P z\|^{2}+\left(L_{\mu} P z, z\right)=\Gamma\|z\|^{2}+\left(\left[L_{\mu}-\Gamma I\right] P z, z\right) \\
& =\Gamma\|z\|^{2}+\langle K z, z\rangle_{X}
\end{aligned}
$$


where $K=R^{-1} \mathfrak{I}\left[L_{\mu}-\Gamma I\right] P: X \rightarrow X$ has rank $d+M(L)$. Also, for all $z, w \in X$,

$$
\begin{aligned}
\langle K z, w\rangle_{X} & =\left(\left[L_{\mu}-\Gamma I\right] P z, w\right)=\left(P\left[L_{\mu}-\Gamma I\right] z, w\right) \\
& =\left(z,\left[L_{\mu}-\Gamma I\right] P w\right)=\langle z, K w\rangle_{X} .
\end{aligned}
$$

This shows that $K$ is self-adjoint for the Hilbert space $\left(X,\langle\cdot, \cdot\rangle_{X}\right)$ and so also $K \in B(X, X)$. Hence $K: X \rightarrow X$ is compact. Since $D\left(L_{\mu}\right)$ is dense in $X$, the continuity of $S_{\mu}$ and $K$ implies that

$$
\left\langle\left[S_{\mu}-K\right] z, z\right\rangle_{X} \geq \Gamma\|z\|^{2} \text { for all } z \in X,
$$

whereas $(\mathrm{G})$ implies that

$$
\left\langle S_{\mu} z, z\right\rangle_{X} \geq \varepsilon\|z\|_{X}^{2}-C\|z\|^{2} \text { for all } z \in X .
$$

Hence

$$
\begin{aligned}
\left\langle\left(1+\frac{\Gamma}{C}\right) S_{\mu} z-K z, z\right\rangle_{X} & \geq \frac{\varepsilon \Gamma}{C}\|z\|_{X}^{2} \text { for all } z \in X, \text { that is } \\
\left\langle S_{\mu} z-K_{1} z, z\right\rangle_{X} & \geq \frac{\varepsilon \Gamma}{\Gamma+C}\|z\|_{X}^{2} \text { where } K_{1}=\frac{C}{\Gamma+C} K .
\end{aligned}
$$

Thus, for any $\lambda \leq \frac{\varepsilon \Gamma}{2(\Gamma+C)}$, we now have that

$$
\left\langle\left[S_{\mu}-\lambda I-K_{1}\right] z, z\right\rangle_{X} \geq \frac{\varepsilon \Gamma}{2(\Gamma+C)}\|z\|_{X}^{2} \text { for all } z \in X .
$$

Since $S_{\mu}-\lambda I-K_{1}: X \rightarrow X$ is self-adjoint, this means that that $S_{\mu}-\lambda I-$ $K_{1}: X \rightarrow X$ is an isomorphism. But $K_{1}: X \rightarrow X$ has rank $d+M\left(L_{\mu}\right)$ and so $S_{\mu}-\lambda I: X \rightarrow X$ is a Fredholm operator for all $\lambda \leq \frac{\varepsilon \Gamma}{2(\Gamma+C)}$. This proves that $\inf \sigma_{e}\left(S_{\mu}\right)>\frac{\varepsilon \Gamma}{2(\Gamma+C)}>0$.

Furthermore, $Q(X) \subset X$ since $P(X) \subset P(H) \subset D\left(L_{\mu}\right) \subset X$. But $K_{1} z=0$ if $P z=0$, so we see that for $\lambda_{1}=\frac{\varepsilon \Gamma}{2(\Gamma+C)}$,

$$
\left\langle\left[S_{\mu}-\lambda_{1} I\right] z, z\right\rangle_{X} \geq \frac{\varepsilon \Gamma}{2(\Gamma+C)}\|z\|_{X}^{2} \text { for all } z \in Q(X)
$$

where $Q(X)$ has codimension $d+M(L)$ in $X$. Thus $M\left(S_{\mu}-\lambda_{1} I\right) \leq d+$ $M\left(L_{\mu}\right)$. Since $\lambda_{1}>0$ we have that $M\left(S_{\mu}\right)+\operatorname{dim} \operatorname{ker} S_{\mu} \leq M\left(S_{\mu}-\lambda_{1} I\right)$. But we already know that $\operatorname{ker} S_{\mu}=\operatorname{ker} L_{\mu}$ so $\operatorname{dim} \operatorname{ker} S_{\mu}=d$ and hence $M\left(S_{\mu}\right) \leq M\left(L_{\mu}\right)$. On the other hand,

$$
\left\langle S_{\mu} z, z\right\rangle_{X}=\left(L_{\mu} z, z\right)<0 \text { for all } z \in V \backslash\{0\}
$$


where $V$ is the span of all the eigenvectors of $L_{\mu}$ associated with its negative eigenvalues. It follows that $M\left(S_{\mu}\right) \geq \operatorname{dim} V=M\left(L_{\mu}\right)$. Thus $M\left(S_{\mu}\right)=$ $M\left(L_{\mu}\right)$.

Corollary 5.5. Suppose that there exist $\mu \in \mathbb{R} \backslash\{0\}$ and $\xi_{\mu} \in X$ such that

$$
A \xi_{\mu} \neq 0, D_{u} G_{\mu}\left(\xi_{\mu}\right)=0
$$

and that $D_{v v}^{2} G_{\mu}\left(\xi_{\mu}\right)$ has the property $(\mathrm{G})$. Then $A \xi_{\mu} \in D\left(L_{\mu}\right)$ and the condition $(\mathrm{B})$ is satisfied provided that

$$
\inf \sigma_{e}\left(L_{\mu}\right)>0, M\left(L_{\mu}\right)=1 \text { and } \operatorname{dim} \operatorname{ker} L_{\mu}=1 .
$$

This follows immediately from Lemma 5.4 since we have $D_{v v}^{2} G_{\mu}\left(\xi_{\mu}\right) A \xi_{\mu}=0$ by (2.4) and so $A \xi_{\mu} \in \operatorname{ker}\left\{S_{\mu}\right\}$.

\section{Constrained minimization and stability}

To find standing waves we must solve the equation (3.1):

$$
H^{\prime}(\xi)-\lambda Q^{\prime}(\xi)=0 \text { for } \lambda \in \mathbb{R} \text { and } \xi \in X \backslash\{0\} .
$$

One approach is to consider $\lambda$ as a Lagrange multiplier for the problem

$$
m(c)=\inf \{H(v): v \in S(c)\}
$$

where $c \neq 0$ is fixed and $S(c)=\{v \in X: Q(v)=c\} \neq \emptyset$. Note that $\left\langle Q^{\prime}(v), v\right\rangle=2 Q(v)=2 c \neq 0$ for all $v \in S(c)$. This ensures that, if $v_{c} \in S(c)$ is such that $H\left(v_{c}\right)=m(c)$, then there exists $\lambda_{c} \in \mathbb{R}$ such that

$$
H^{\prime}\left(v_{c}\right)-\lambda_{c} Q^{\prime}\left(v_{c}\right)=0 .
$$

Since $c=Q\left(v_{c}\right)=\frac{1}{2}\left\langle B v_{c}, v_{c}\right\rangle=\frac{1}{2}\left(v_{c}, J A v_{c}\right)$, we have that $A v_{c} \neq 0$. Also,

$$
\left\langle H^{\prime}\left(v_{c}\right), v_{c}\right\rangle=\lambda_{c}\left\langle Q^{\prime}\left(v_{c}\right), v_{c}\right\rangle=2 \lambda_{c} Q\left(v_{c}\right)=2 c \lambda_{c},
$$

so that $\lambda_{c} \neq 0$ if $\left\langle H^{\prime}\left(v_{c}\right), v_{c}\right\rangle \neq 0$ and in this case $\left(\lambda_{c}, v_{c}\right)$ generates a (non-stationary) standing wave.

However, under the hypothesis $(\mathrm{H})$, it may be that $S(c) \neq \emptyset$ but the infimum in (6.1) is not attained. To address this problem we introduce the following condition.

$(\mathrm{M})_{c} S(c) \neq \emptyset$ and any sequence $\left\{v_{n}\right\} \subset X$ having the properties

$$
H\left(v_{n}\right) \rightarrow m(c) \text { and } Q\left(v_{n}\right) \rightarrow c
$$

contains a subsequence converging in $X$. 
In the context of the nonlinear Schrödinger equation, Section 8.5 deals with situations where $(\mathrm{M})_{c}$ is satisfied for at least some $c \neq 0$.

Proposition 6.1. Let $c \neq 0$ be such that $(\mathrm{M})_{c}$ is satisfied. Then there exists $v_{c} \in S(c)$ such that $H\left(v_{c}\right)=m(c)$. Furthermore, there exists $\lambda_{c} \in \mathbb{R}$ such that $D_{v} G_{\lambda_{c}}\left(v_{c}\right)=0$ and

$$
\left\langle D_{v v}^{2} G_{\lambda_{c}}\left(v_{c}\right) w, w\right\rangle \geq 0 \text { for all } w \in X \text { such that }\left\langle B v_{c}, w\right\rangle=0 .
$$

Remark. Since $D_{v v}^{2} G_{\lambda_{c}}\left(v_{c}\right) A v_{c}=0$ and $\left\langle B v_{c}, w\right\rangle=\left\langle R^{-1} B v_{c}, w\right\rangle_{X}$, it follows from (6.2) that

$$
\left\langle D_{v v}^{2} G_{\lambda_{c}}\left(v_{c}\right) z, z\right\rangle \geq 0 \text { for all } z \in\left\{A v_{c}, R^{-1} B v_{c}\right\}^{\perp} .
$$

Setting $\lambda=\lambda_{c}$ and $\xi_{\lambda}=v_{c}$, we see that the stability criterion (SC) holds if and only if $A \xi_{\lambda}$ is the only degenerate direction for $D_{v v}^{2} G_{\lambda}\left(\xi_{\lambda}\right)$ in $\left\{R^{-1} B v_{c}\right\}^{\perp}$. That is, for a minimizer $v_{c}$ of (6.1), the condition (SC) is satisfied if and only if

$$
\operatorname{ker} D_{v v}^{2} G_{\lambda_{c}}\left(v_{c}\right)=\operatorname{span}\left\{A \xi_{\lambda}\right\} .
$$

The geometrical significance of $\left\{R^{-1} B v_{c}\right\}^{\perp}$ will emerge from the proof.

Proof. There exists a sequence $\left\{v_{n}\right\} \subset S(c)$ such that $H\left(v_{n}\right) \rightarrow m(c)$. By $(\mathrm{M})_{c}$ there exist $v_{c} \in X$ and a subsequence $\left\{v_{n_{k}}\right\}$ such that $v_{n_{k}} \rightarrow v_{c}$ in $X$. From the continuity of $H$ and $Q$, it follows that $v_{c} \in S(c)$ such that $H\left(v_{c}\right)=m(c)$.

Next we observe that since $\left\langle Q^{\prime}(v), v\right\rangle=2 c \neq 0$ for $v \in S(c), S(c)$ is a smooth co-dimension 1 manifold and the tangent space at $v$ is

$$
T_{v}=\left\{w \in X:\left\langle Q^{\prime}(v), w\right\rangle=0\right\} .
$$

Given $v \in S(c)$ and $w \in T_{v}$, we define $r:(-\delta, \delta) \rightarrow \mathbb{R}$ by

$$
r(t)=\left\{\frac{Q(v)}{Q(v)+t^{2} Q(w)}\right\}^{1 / 2}
$$

where $\delta>0$ is chosen so that $Q(v)+t^{2} Q(w)=c+t^{2} Q(w) \neq 0$ for all $t \in(-\delta, \delta)$. Then we define $\varphi:(-\delta, \delta) \rightarrow X$ by

$$
\varphi(t)=r(t)\{v+t w\} .
$$

Now $Q(\varphi(t))=\frac{1}{2}\langle B \varphi(t), \varphi(t)\rangle$ and so

$$
Q(\varphi(t))=r(t)^{2}\left\{Q(v)+t^{2} Q(w)\right\} \text { since }\langle B v, w\rangle=\left\langle Q^{\prime}(v), w\right\rangle=0 \text { for } w \in T_{v} .
$$


Hence $Q(\varphi(t))=Q(v)=c$, showing that $\varphi(t) \in S(c)$ for all $t \in(-\delta, \delta)$. We also have that

$$
\varphi(0)=v, \varphi^{\prime}(0)=w \text { and } \varphi^{\prime \prime}(0)=r^{\prime \prime}(0) v=-\frac{Q(w)}{Q(v)} v .
$$

By the minimality of $v_{c}$, this implies that $\left.\frac{d^{2}}{d t^{2}} H(\varphi(t))\right|_{t=0} \geq 0$ if we choose $v=v_{c}$ and $w \in T_{v_{c}}$. But

$$
\begin{aligned}
& \left.\frac{d^{2}}{d t^{2}} H(\varphi(t))\right|_{t=0}=\left\langle H^{\prime \prime}(\varphi(0)) \varphi^{\prime}(0), \varphi^{\prime}(0)\right\rangle+\left\langle H^{\prime}(\varphi(0)), \varphi^{\prime \prime}(0)\right\rangle \\
& =\left\langle H^{\prime \prime}\left(v_{c}\right) w, w\right\rangle-\frac{Q(w)}{Q\left(v_{c}\right)}\left\langle H^{\prime}\left(v_{c}\right), v_{c}\right\rangle=\left\langle H^{\prime \prime}\left(v_{c}\right) w, w\right\rangle-\frac{Q(w)}{Q\left(v_{c}\right)} \lambda_{c}\left\langle Q^{\prime}\left(v_{c}\right), v_{c}\right\rangle \\
& =\left\langle H^{\prime \prime}\left(v_{c}\right) w, w\right\rangle-\lambda_{c}\langle B w, w\rangle=\left\langle D_{v v}^{2} G_{\lambda_{c}}\left(v_{c}\right) w, w\right\rangle .
\end{aligned}
$$

This proves that

$$
\left\langle D_{v v}^{2} G_{\lambda_{c}}\left(v_{c}\right) w, w\right\rangle \geq 0 \text { for all } w \in X \text { such that }\left\langle B v_{c}, w\right\rangle=0 .
$$

Remark. We note that $T_{v_{c}}=\left\{R^{-1} B \xi_{\lambda}\right\}^{\perp}$ and that $A \xi_{\lambda} \in T_{v_{c}}$. Thus (SC) is satisfied by a minimizer of (6.1) if and only if

$$
T_{v_{c}} \cap \operatorname{ker} D_{v v}^{2} G_{\lambda_{c}}\left(v_{c}\right)=\operatorname{span}\left\{A \xi_{\lambda}\right\} .
$$

\subsection{A weaker notion of stability}

In fact, even if (SC) does not hold the standing wave generated by a constrained minimizer can still be stable in a certain sense. For $c \neq 0$ such that $S(c) \neq \emptyset$, let

$$
M(c)=\{v \in S(c): H(v)=m(c)\}
$$

and, for $v \in M(c)$, let $\lambda_{v}$ denote the associated Lagrange multiplier such that $H^{\prime}(v)=\lambda_{v} Q^{\prime}(v)$. We say that the standing wave $\varphi(t)=T\left(\lambda_{v} t\right) v$ generated by $v \in M(c)$ is weakly stable when it has the following property.

(WS) The condition (GWP at $v$ ) holds and, for any $\varepsilon>0$, there exists $\delta>0$ such that, for all $u_{0} \in B(v, \delta)$,

$$
d\left(u(t), M((c))=\inf \left\{\|u(t)-v\|_{X}: v \in M(c)\right\}<\varepsilon \text { for all } t \geq 0,\right.
$$

where $u$ is the solution of (IVP) with $u(0)=u_{0}$.

Since the orbit $\Theta(v)$ is a subset of $M(c), \varphi(t) \in M(c)$ for all $t \in \mathbb{R}$ and it is clear that (WS) is a weaker property than true orbital stability. In some special cases (see [30]), one can show that $\Theta(v)=M(c)$ and then (WS) is equivalent to orbital stability. More generally, if $d(\Theta(v), M(c) \backslash \Theta(v))>0$ then orbital stability of $\varphi(t)$ follows from (WS). 
Theorem 6.2. Let $c \neq 0$ be such that $(\mathrm{M})_{c}$ is satisfied. Then $M(c) \neq \emptyset$ and, for any $v \in M(c)$ there exists $\lambda_{v} \in \mathbb{R}$ such that $H^{\prime}(v)=\lambda_{v} Q^{\prime}(v)$. The associated standing wave $\varphi(t)=T\left(\lambda_{v} t\right) v$ has the property (WS) provided that (GWP at $v$ ) holds.

Proof. By Proposition 6.1 we have that $M(c) \neq \emptyset$. Let $v \in M(c)$. If (GWP at $v$ ) holds and (WS) is not satisfied, there exist $\varepsilon>0$ and sequences $\left\{w_{n}\right\} \subset X$ and $\left\{t_{n}\right\} \subset(0, \infty)$ such that

$$
\left\|w_{n}-v\right\|_{X} \rightarrow 0 \text { and } d\left(u\left(t_{n}\right), M(c)\right) \geq \varepsilon
$$

for all $n \in \mathbb{N}$, where $u_{n}$ is the solution of (IVP) with initial condition $w_{n}$. But

$$
H\left(u\left(t_{n}\right)\right)=H\left(w_{n}\right) \rightarrow H(v) \text { and } Q\left(u\left(t_{n}\right)\right)=Q\left(w_{n}\right) \rightarrow Q(v)
$$

so it follows from $(\mathrm{M})_{c}$ that there exist $z \in X$ and a subsequence such that $u\left(t_{n_{k}}\right) \rightarrow z$ in $X$. Then $H(z)=H(v)=m(c)$ and $Q(z)=Q(v)=c$, showing that $z \in M(c)$. Hence

$$
d\left(u\left(t_{n_{k}}\right), M(c)\right) \leq\left\|u\left(t_{n_{k}}\right)-z\right\|_{X}
$$

and, for $k$ large enough, $\left\|u\left(t_{n_{k}}\right)-z\right\|_{X}<\varepsilon / 2$, contradicting the choice of $\varepsilon$ and the sequences $\left\{w_{n}\right\}$ and $\left\{t_{n}\right\}$.

Let us mention one useful variant of this simple argument when $H$ and $Q$ are invariant with respect to an additional group of isometries. Let $O(X)$ denote the group (with respect to composition) of all isometric isomorphisms of $X$.

(E) There is a subgroup $\Upsilon$ of $O(X)$ such that

$$
H(\gamma v)=H(v) \text { and } Q(\gamma v)=Q(v) \text { for all } v \in X \text { and } \gamma \in \Upsilon \text {. }
$$

Under this assumption the sets $S(c)$ and $M(c)$ are $\Upsilon$-invariant. In this context we can use a weaker form of $(\mathrm{M})_{c}$.

$(\mathrm{EM})_{c} S(c) \neq \emptyset$ and, for any sequence $\left\{v_{n}\right\} \subset X$ having the properties

$$
H\left(v_{n}\right) \rightarrow m(c) \text { and } Q\left(v_{n}\right) \rightarrow c
$$

we can extract a subsequence $\left\{v_{n_{k}}\right\}$ and find elements $\gamma_{n_{k}} \in \Upsilon$ such that $\left\{\gamma_{n_{k}}\left(v_{n_{k}}\right)\right\}$ converges in $X$.

Corollary 6.3. In addition to the usual hypothesis $(\mathrm{H})$, suppose that $(\mathrm{E})$ holds. Let $c \neq 0$ be such that $(\mathrm{EM})_{c}$ is satisfied. Then $M(c) \neq \emptyset$. Furthermore, for any $v \in M(c)$ there exists $\lambda_{v} \in \mathbb{R}$ such that $H^{\prime}(v)=\lambda_{v} Q^{\prime}(v)$. 
If (GWP at $v$ ) holds, the associated standing wave $\varphi(t)=T\left(\lambda_{v} t\right) v$ has the property (WS).

Proof. Let $\left\{v_{n}\right\} \subset S(c)$ be such that $H\left(v_{n}\right) \rightarrow m(c)$. By $(\mathrm{EM})_{c}$ there exist $v \in X$, a subsequence $\left\{v_{n_{k}}\right\}$ and $\gamma_{n_{k}} \in \Upsilon$ such that $\gamma_{n_{k}}\left(v_{n_{k}}\right) \rightarrow v$ in $X$. But

$$
\begin{aligned}
& H(v)=\lim _{k \rightarrow \infty} H\left(\gamma_{n_{k}}\left(v_{n_{k}}\right)\right)=\lim _{k \rightarrow \infty} H\left(v_{n_{k}}\right)=m(c) \text { and } \\
& Q(v)=\lim _{k \rightarrow \infty} Q\left(\gamma_{n_{k}}\left(v_{n_{k}}\right)\right)=\lim _{k \rightarrow \infty} Q\left(v_{n_{k}}\right)=c
\end{aligned}
$$

so $v \in M(c)$.

If (GWP at $v$ ) holds but not (WS), there exist $\varepsilon>0$ and sequences $\left\{w_{n}\right\} \subset X$ and $\left\{t_{n}\right\} \subset(0, \infty)$ such that

$$
\left\|w_{n}-v\right\|_{X} \rightarrow 0 \text { and } d\left(u\left(t_{n}\right), M(c)\right) \geq \varepsilon
$$

for all $n \in \mathbb{N}$ where $u_{n}$ is the solution of (IVP) with initial condition $w_{n}$. Since

$$
H\left(u_{n}\left(t_{n}\right)\right)=H\left(w_{n}\right) \rightarrow H(v) \text { and } Q\left(u_{n}\left(t_{n}\right)\right)=Q\left(w_{n}\right) \rightarrow Q(v)
$$

so it follows from $(\mathrm{EM})_{c}$ that there exist $z \in X$, a subsequence $\left\{u_{n_{k}}\left(t_{n_{k}}\right)\right\}$ and elements $\gamma_{n_{k}} \in \Upsilon$ such that $\gamma_{n_{k}} u_{n_{k}}\left(t_{n_{k}}\right) \rightarrow z$ in $X$. Then $H(z)=$ $H(v)=m(c)$ and $Q(z)=Q(v)=c$, showing that $z \in M(c)$. Hence, for all $k, \gamma_{n_{k}}^{-1} z \in M(c)$ and

$$
d\left(u_{n_{k}}\left(t_{n_{k}}\right), M(c)\right) \leq\left\|u_{n_{k}}\left(t_{n_{k}}\right)-\gamma_{n_{k}}^{-1} z\right\|_{X}=\left\|\gamma_{n_{k}} u_{n_{k}}\left(t_{n_{k}}\right)-z\right\|_{X} .
$$

For $k$ large enough, $\left\|\gamma_{n_{k}} u_{n_{k}}\left(t_{n_{k}}\right)-z\right\|_{X}<\varepsilon / 2$, contradicting the choice of $\varepsilon$ and the sequences $\left\{w_{n}\right\}$ and $\left\{t_{n}\right\}$. Thus property (WS) must hold if (GWP at $v)$ is satisfied.

\section{The nonlinear Schrödinger equation}

We consider the nonlinear Schrödinger equation in the following form

$$
i \partial_{t} w(x, t)+\Delta w(x, t)+f\left(x,|w(x, t)|^{2}\right) w(x, t)=0
$$

where $w: \mathbb{R}^{N} \times[0, \infty) \rightarrow \mathbb{C}$ and $f: \mathbb{R}^{N} \times[0, \infty) \rightarrow \mathbb{R}$

Let $w=\varphi+i \psi \longmapsto\left(\begin{array}{c}\varphi \\ \psi\end{array}\right)$. Then

$$
i \partial_{t} w(x, t)=-\partial_{t} \psi+i \partial_{t} \varphi \mapsto\left(\begin{array}{c}
-\partial_{t} \psi \\
\partial_{t} \varphi
\end{array}\right)=\left[\begin{array}{cc}
0 & -1 \\
1 & 0
\end{array}\right]\left(\begin{array}{c}
\partial_{t} \varphi \\
\partial_{t} \psi
\end{array}\right) .
$$


Also

$$
\Delta w(x, t)+f\left(x,|w(x, t)|^{2}\right) w(x, t) \mapsto\left(\begin{array}{c}
\Delta \varphi+f\left(x, \varphi^{2}+\psi^{2}\right) \varphi \\
\Delta \psi+f\left(x, \varphi^{2}+\psi^{2}\right) \psi
\end{array}\right)
$$

so (CNLS) is

$$
\left[\begin{array}{cc}
0 & -1 \\
1 & 0
\end{array}\right]\left(\begin{array}{c}
\partial_{t} \varphi \\
\partial_{t} \psi
\end{array}\right)=-\left(\begin{array}{c}
\Delta \varphi+f\left(x, \varphi^{2}+\psi^{2}\right) \varphi \\
\Delta \psi+f\left(x, \varphi^{2}+\psi^{2}\right) \psi
\end{array}\right) .
$$

Let

$$
F(x, s)=\int_{0}^{s} f(x, \sigma) d \sigma
$$

and consider formally (at this point) the Hamiltonian defined by

$$
H\left(\left(\begin{array}{c}
\varphi \\
\psi
\end{array}\right)\right)=\frac{1}{2} \int_{\mathbb{R}^{N}}|\nabla \varphi|^{2}+|\nabla \psi|^{2}-F\left(x, \varphi^{2}+\psi^{2}\right) d x .
$$

Then

$$
H^{\prime}\left(\left(\begin{array}{c}
\varphi \\
\psi
\end{array}\right)\right)=\left(\begin{array}{c}
-\Delta \varphi-f\left(x, \varphi^{2}+\psi^{2}\right) \varphi \\
-\Delta \psi-f\left(x, \varphi^{2}+\psi^{2}\right) \psi
\end{array}\right)
$$

so (CNLS) becomes

$$
\left(\begin{array}{c}
\partial_{t} \varphi \\
\partial_{t} \psi
\end{array}\right)=\left[\begin{array}{cc}
0 & 1 \\
-1 & 0
\end{array}\right] H^{\prime}\left(\left(\begin{array}{c}
\varphi \\
\psi
\end{array}\right)\right)
$$

The equation (CNLS) arises in many contexts. One example is in the study of guided waves propagating through a non-linear optical material. In this context, a derivation of (CNLS) from first principles and an interpretation of standing waves and their orbital stability is given in [31]. See equation (7.9) of [31] for (CNLS) and then Section 7.2 of the same paper for a situation leading to the hypotheses introduced in Section 8.3 of these notes.

We observe also that any nonlinearity that is equivariant with respect to rotations about the origin in $\mathbb{C}$ can be expressed in the form $f\left(x,|w|^{2}\right) w$ appearing in (CNLS). Indeed, if $h: \mathbb{R}^{N} \times \mathbb{C} \rightarrow \mathbb{C}$ is such that

$$
h\left(x, e^{i \theta} z\right)=e^{i \theta} h(x, z) \text { for all } \theta \in \mathbb{R} \text { and } z \in \mathbb{C},
$$

then clearly $h(x, 0)=0$ and, setting

$$
f(x, s)=\frac{h(x, \sqrt{s})}{\sqrt{s}} \text { for } x \in \mathbb{R}^{N} \text { and } s>0,
$$

we find that

$$
h(x, z)=h\left(x,|z| e^{i \arg z}\right)=e^{i \arg z} h(x,|z|)=f\left(x,|z|^{2}\right) z \text { for all } z \in \mathbb{C} \backslash\{0\} .
$$


As a result of this equivariance, $w(x, t) \equiv 0$ is always a stationary solution of (CNLS).

In this context, we observe that the requirement that $f: \mathbb{R}^{N} \times[0, \infty) \rightarrow$ $\mathbb{R}$ in (CNLS) corresponds to the property $h(x, s) \in \mathbb{R}$ for all $x \in \mathbb{R}^{N}$ and $s \in[0, \infty)$.

\subsection{Expressing (CNLS) in the form (2.1)}

We now reformulate (CNLS) as a special case of (2.1) in the appropriate function spaces.

$$
\begin{aligned}
X & =H^{1}\left(\mathbb{R}^{N}\right) \times H^{1}\left(\mathbb{R}^{N}\right), \\
H & =L^{2}\left(\mathbb{R}^{N}\right) \times L^{2}\left(\mathbb{R}^{N}\right), \\
X^{*} & =H^{-1}\left(\mathbb{R}^{N}\right) \times H^{-1}\left(\mathbb{R}^{N}\right),
\end{aligned}
$$

with the usual scalar products:

$$
\begin{aligned}
& \left\langle\left(\begin{array}{c}
\varphi \\
\psi
\end{array}\right),\left(\begin{array}{c}
\xi \\
\eta
\end{array}\right)\right\rangle_{X}=\int_{\mathbb{R}^{N}} \nabla \varphi \cdot \nabla \xi+\nabla \psi \cdot \nabla \eta+\varphi \xi+\psi \eta d x \\
& \left(\left(\begin{array}{c}
\varphi \\
\psi
\end{array}\right),\left(\begin{array}{c}
\xi \\
\eta
\end{array}\right)\right)=\int_{\mathbb{R}^{N}} \varphi \xi+\psi \eta d x .
\end{aligned}
$$

It is easily verified that, in this case, the operator $S=R^{-1} \mathfrak{I}: X \rightarrow X$ is given by $S=(-\Delta+1)^{-1}$.

Define $J: X \rightarrow X$ and $A: X \rightarrow X$ by

$$
J\left(\begin{array}{c}
\varphi \\
\psi
\end{array}\right)=\left(\begin{array}{c}
-\psi \\
\varphi
\end{array}\right) \text { and } A=-J=\left[\begin{array}{cc}
0 & I \\
-I & 0
\end{array}\right]
$$

where $I: X \rightarrow X$ is the identity. Then

$$
T(\theta)=e^{\theta A}=e^{-\theta J}=\left[\begin{array}{cc}
\cos \theta I & \sin \theta I \\
-\sin \theta I & \cos \theta I
\end{array}\right] .
$$

Let $F: \mathbb{R}^{N} \times[0, \infty) \rightarrow \mathbb{R}$ be such that

$$
\Phi(u)=\int_{\mathbb{R}^{N}} F\left(x, u^{2}\right) d x
$$

defines a functional $\Phi \in C^{2}\left(H^{1}\left(\mathbb{R}^{N}\right), \mathbb{R}\right)$ with

$$
\begin{aligned}
\left\langle\Phi^{\prime}(u), v\right\rangle & =2 \int_{\mathbb{R}^{N}} f\left(x, u^{2}\right) u v d x \text { and } \\
\left\langle\Phi^{\prime \prime}(u) z, v\right\rangle & =2 \int_{\mathbb{R}^{N}}\left\{2 \partial_{s} f\left(x, u^{2}\right) u^{2}+f\left(x, u^{2}\right)\right\} z v d x .
\end{aligned}
$$


(See the beginning of Section 8 for an example of a function $F$ having these properties.) For $H: X \rightarrow \mathbb{R}$ defined by (7.1) we then have that $H \in C^{2}(X, \mathbb{R})$ with

$$
\begin{aligned}
& \left\langle H^{\prime}\left(\left(\begin{array}{l}
\varphi \\
\psi
\end{array}\right)\right),\left(\begin{array}{l}
v \\
z
\end{array}\right)\right\rangle \\
& =\int_{\mathbb{R}^{N}} \nabla \varphi \cdot \nabla v+\nabla \psi \cdot \nabla z-f\left(x, \varphi^{2}+\psi^{2}\right) \varphi v-f\left(x, \varphi^{2}+\psi^{2}\right) \psi z d x .
\end{aligned}
$$

For $\varphi, \psi \in C_{0}^{\infty}\left(\mathbb{R}^{N}\right)$,

$$
\begin{aligned}
& \left\langle H^{\prime}\left(\left(\begin{array}{c}
\varphi \\
\psi
\end{array}\right)\right),\left(\begin{array}{c}
v \\
z
\end{array}\right)\right\rangle \\
& =-\int_{\mathbb{R}^{N}}\left[\Delta \varphi+f\left(x, \varphi^{2}+\psi^{2}\right) \varphi\right] v+\left[\Delta \psi+f\left(x, \varphi^{2}+\psi^{2}\right) \psi\right] z d x \\
& =-\left\langle\left(\begin{array}{c}
\Im\left[\Delta \varphi+f\left(x, \varphi^{2}+\psi^{2}\right) \varphi\right] \\
\Im\left[\Delta \psi+f\left(x, \varphi^{2}+\psi^{2}\right) \psi\right]
\end{array}\right),\left(\begin{array}{c}
v \\
z
\end{array}\right)\right\rangle
\end{aligned}
$$

so that we can write

$$
H^{\prime}\left(\left(\begin{array}{c}
\varphi \\
\psi
\end{array}\right)\right)=-\mathfrak{I}\left(\begin{array}{c}
\Delta \varphi+f\left(x, \varphi^{2}+\psi^{2}\right) \varphi \\
\Delta \psi+f\left(x, \varphi^{2}+\psi^{2}\right) \psi
\end{array}\right)
$$

when convenient by interpreting the right hand side as a continuous extension from $C_{0}^{\infty}\left(\mathbb{R}^{N}\right) \times C_{0}^{\infty}\left(\mathbb{R}^{N}\right)$ to $X$ with values in $X^{*}$. Noting that

$$
\begin{aligned}
& \left\langle H^{\prime}\left(\left(\begin{array}{c}
\varphi \\
\psi
\end{array}\right)\right), A\left(\begin{array}{c}
\varphi \\
\psi
\end{array}\right)\right\rangle=\left\langle H^{\prime}\left(\left(\begin{array}{c}
\varphi \\
\psi
\end{array}\right)\right),\left(\begin{array}{c}
\psi \\
-\varphi
\end{array}\right)\right\rangle \\
& =\int_{\mathbb{R}^{N}} \nabla \varphi \cdot \nabla \psi-\nabla \psi \cdot \nabla \varphi-f\left(x, \varphi^{2}+\psi^{2}\right) \varphi \psi+f\left(x, \varphi^{2}+\psi^{2}\right) \psi \varphi d x \\
& =0,
\end{aligned}
$$

we see that our hypothesis $(\mathrm{H})$ is satisfied and

$$
\frac{d}{d t} \mathfrak{I}\left(\begin{array}{c}
\varphi \\
\psi
\end{array}\right)=J^{*} H^{\prime}\left(\left(\begin{array}{c}
\varphi \\
\psi
\end{array}\right)\right.
$$

can be taken as a weak form of (CNLS). Indeed, for a solution $u=\left(\begin{array}{c}\varphi \\ \psi\end{array}\right)$ : $\left(-b_{-}, b_{+}\right) \rightarrow X$ of (NLS), we have that $u \in C\left(\left(-b_{-}, b_{+}\right), X\right)$, $\Im u \in C^{1}\left(\left(-b_{-}, b_{+}\right), X^{*}\right)$ and for any $\left(\begin{array}{c}v \\ z\end{array}\right) \in C_{0}^{\infty}\left(\mathbb{R}^{N}\right) \times C_{0}^{\infty}\left(\mathbb{R}^{N}\right)$ and 
$t \in\left(-b_{-}, b_{+}\right)$,

$$
\begin{aligned}
& \left\langle\frac{d}{d t} \mathfrak{I}\left(\begin{array}{c}
\varphi \\
\psi
\end{array}\right)(t), J\left(\begin{array}{c}
v \\
z
\end{array}\right)\right\rangle \\
& =\frac{d}{d t}\left\langle\mathfrak{I}\left(\begin{array}{c}
\varphi \\
\psi
\end{array}\right)(t), J\left(\begin{array}{c}
v \\
z
\end{array}\right)\right\rangle=\frac{d}{d t} \int_{\mathbb{R}^{N}}-\varphi(t) z+\psi(t) v d x
\end{aligned}
$$

whereas

$$
\begin{aligned}
& \left\langle J^{*} H^{\prime}\left(\left(\begin{array}{c}
\varphi \\
\psi
\end{array}\right)\right)(t), J\left(\begin{array}{l}
v \\
z
\end{array}\right)\right\rangle=\left\langle H^{\prime}\left(\left(\begin{array}{c}
\varphi \\
\psi
\end{array}\right)\right)(t), J^{2}\left(\begin{array}{l}
v \\
z
\end{array}\right)\right\rangle \\
& =-\int_{\mathbb{R}^{N}} \nabla \varphi(t) \cdot \nabla v+\nabla \psi(t) \cdot \nabla z-f\left(x,|w(t)|^{2}\right) \varphi(t) v \\
& \quad-f\left(x,|w(t)|^{2}\right) \psi(t) z d x .
\end{aligned}
$$

where $w=\varphi+i \psi \in C\left(\left(-b_{-}, b_{+}\right), H^{1}\left(\mathbb{R}^{N}, \mathbb{C}\right)\right) \cap C^{1}\left(\left(-b_{-}, b_{+}\right), H^{-1}\left(\mathbb{R}^{N}, \mathbb{C}\right)\right)$. It follows that, for all $v, z \in C_{0}^{\infty}\left(\mathbb{R}^{N}\right)$ and $t \in\left(-b_{-}, b_{+}\right)$,

$$
\begin{aligned}
& \frac{d}{d t} \int_{\mathbb{R}^{N}} \varphi(t) z-\psi(t) v d x= \\
& \int_{\mathbb{R}^{N}} \nabla \varphi(t) \cdot \nabla v+\nabla \psi(t) \cdot \nabla z-f\left(x,|w(t)|^{2}\right) \varphi(t) v-f\left(x,|w(t)|^{2}\right) \psi(t) z d x .
\end{aligned}
$$

From this identity we deduce that

$$
\frac{d}{d t} \int_{\mathbb{R}^{N}} i w(t) \varsigma d x=\int_{\mathbb{R}^{N}} \nabla w(t) \cdot \nabla \varsigma-f\left(x,|w(t)|^{2}\right) w(t) \varsigma d x
$$

for all $\varsigma \in C_{0}^{\infty}\left(\mathbb{R}^{N}, \mathbb{C}\right)$ which is a weak form of (CNLS).

Returning to (NLS), a standing wave has the form

$$
\begin{gathered}
T(\lambda t)\left(\begin{array}{c}
\varphi \\
\psi
\end{array}\right)=\left(\begin{array}{c}
\varphi \cos \lambda t+\psi \sin \lambda t \\
-\varphi \sin \lambda t+\psi \cos \lambda t
\end{array}\right) \text { where } \\
\left(\begin{array}{c}
\varphi \\
\psi
\end{array}\right) \in X=H^{1}\left(\mathbb{R}^{N}\right) \times H^{1}\left(\mathbb{R}^{N}\right) .
\end{gathered}
$$

It is a time periodic (in fact time harmonic) solution of (NLS). In complex notation, it corresponds to a solution of (CNLS) the form

$$
e^{-i \lambda t}\{\varphi(x)+i \psi(x)\} .
$$




\subsection{Solutions of the stationary equation}

Since $J A=-J^{2}=I$, we have that $B=\mathfrak{I}$ and

$$
\begin{aligned}
Q\left(\left(\begin{array}{l}
\varphi \\
\psi
\end{array}\right)\right) & =\frac{1}{2}\left\langle\mathfrak{I}\left(\begin{array}{c}
\varphi \\
\psi
\end{array}\right),\left(\begin{array}{l}
\varphi \\
\psi
\end{array}\right)\right\rangle=\frac{1}{2}\left(\left(\begin{array}{l}
\varphi \\
\psi
\end{array}\right),\left(\begin{array}{l}
\varphi \\
\psi
\end{array}\right)\right) \\
& =\frac{1}{2} \int_{\mathbb{R}^{N}} \varphi^{2}+\psi^{2} d x=\frac{1}{2} \int_{\mathbb{R}^{N}}|w|^{2} d x \text { where } w=\varphi+i \psi .
\end{aligned}
$$

The stationary equation is

$$
H^{\prime}\left(\left(\begin{array}{c}
\varphi \\
\psi
\end{array}\right)\right)=\lambda \Im\left(\begin{array}{c}
\varphi \\
\psi
\end{array}\right)
$$

which we can write as

$$
\mathfrak{I}\left(\begin{array}{c}
\Delta \varphi+f\left(x, \varphi^{2}+\psi^{2}\right) \varphi+\lambda \varphi \\
\Delta \psi+f\left(x, \varphi^{2}+\psi^{2}\right) \psi+\lambda \psi
\end{array}\right)=\left(\begin{array}{l}
0 \\
0
\end{array}\right) .
$$

The augmented Hamiltonian $\left(G_{\lambda}=H-\lambda Q\right)$ is

$$
G_{\lambda}\left(\left(\begin{array}{c}
\varphi \\
\psi
\end{array}\right)\right)=\frac{1}{2} \int_{\mathbb{R}^{N}}|\nabla \varphi|^{2}+|\nabla \psi|^{2}-F\left(x, \varphi^{2}+\psi^{2}\right)-\lambda\left(\varphi^{2}+\psi^{2}\right) d x .
$$

Hence

$$
\begin{aligned}
& D_{u} G_{\lambda}\left(\left(\begin{array}{c}
\varphi \\
\psi
\end{array}\right)\right)=-\mathfrak{I}\left(\begin{array}{c}
\Delta \varphi+f\left(x, \varphi^{2}+\psi^{2}\right) \varphi+\lambda \varphi \\
\Delta \psi+f\left(x, \varphi^{2}+\psi^{2}\right) \psi+\lambda \psi
\end{array}\right) \text { and } \\
& D_{u u}^{2} G_{\lambda}\left(\left(\begin{array}{c}
\varphi \\
\psi
\end{array}\right)\right)\left(\begin{array}{c}
v \\
z
\end{array}\right) \\
& =-\mathfrak{I}\left(\begin{array}{c}
\Delta v+f\left(x, \varphi^{2}+\psi^{2}\right) v+2 \partial_{s} f\left(x, \varphi^{2}+\psi^{2}\right)[\varphi v+\psi z] \varphi+\lambda v \\
\Delta z+f\left(x, \varphi^{2}+\psi^{2}\right) z+2 \partial_{s} f\left(x, \varphi^{2}+\psi^{2}\right)[\varphi v+\psi z] \psi+\lambda z
\end{array}\right) .
\end{aligned}
$$

Suppose that $\xi_{\lambda}$ is a "real" solution of the (SNLS):

$$
\xi_{\lambda}=\left(\begin{array}{c}
\varphi_{\lambda} \\
0
\end{array}\right) \in X \text { with } \mathfrak{I}\left\{\Delta \varphi_{\lambda}+f\left(x, \varphi_{\lambda}^{2}\right) \varphi_{\lambda}+\lambda \varphi_{\lambda}\right\}=0 .
$$

For such a solution, $\varphi_{\lambda} \in H^{1}\left(\mathbb{R}^{N}\right)$ and

$$
\begin{aligned}
& D_{u u}^{2} G_{\lambda}\left(\left(\begin{array}{c}
\varphi_{\lambda} \\
0
\end{array}\right)\right)\left(\begin{array}{c}
v \\
z
\end{array}\right) \\
& =-\mathfrak{I}\left(\begin{array}{c}
\Delta v+f\left(x, \varphi_{\lambda}^{2}\right) v+2 \partial_{s} f\left(x, \varphi_{\lambda}^{2}\right) \varphi_{\lambda}^{2} v+\lambda v \\
\Delta z+f\left(x, \varphi_{\lambda}^{2}\right) z+\lambda z
\end{array}\right)
\end{aligned}
$$


and we can write

$$
\begin{aligned}
& D_{u u}^{2} G_{\lambda}\left(\left(\begin{array}{c}
\varphi_{\lambda} \\
0
\end{array}\right)\right) \\
& =\mathfrak{I}\left[\begin{array}{cc}
-\Delta-f\left(x, \varphi_{\lambda}^{2}\right)-2 \partial_{s} f\left(x, \varphi_{\lambda}^{2}\right) \varphi_{\lambda}^{2}-\lambda & 0 \\
0 & -\Delta-f\left(x, \varphi_{\lambda}^{2}\right)-\lambda
\end{array}\right] .
\end{aligned}
$$

In particular,

$$
D_{u u}^{2} G_{\lambda}\left(\left(\begin{array}{l}
0 \\
0
\end{array}\right)\right)=\mathfrak{I}\left[\begin{array}{cc}
-\Delta-f(x, 0)-\lambda & 0 \\
0 & -\Delta-f(x, 0)-\lambda
\end{array}\right] .
$$

The structure of the Hessian is even clearer if we set $g(x, s)=f\left(x, s^{2}\right) s$ for $s \in \mathbb{R}$. The stationary equation for a real solution becomes

$$
\varphi_{\lambda} \in H^{1}\left(\mathbb{R}^{N}\right) \text { and } \mathfrak{I}\left\{\Delta \varphi_{\lambda}+g\left(x, \varphi_{\lambda}\right)+\lambda \varphi_{\lambda}\right\}=0
$$

and the Hessian of the augmented Hamiltonian becomes

$$
\mathfrak{I}\left[\begin{array}{cc}
-\Delta-\partial_{s} g\left(x, \varphi_{\lambda}\right)-\lambda & 0 \\
0 & -\Delta-\frac{g\left(x, \varphi_{\lambda}\right)}{\varphi_{\lambda}}-\lambda
\end{array}\right] .
$$

Thus, for $v, z \in H^{1}\left(\mathbb{R}^{N}\right)$,

$$
\begin{aligned}
& \left\langle D_{u u}^{2} G_{\lambda}\left(\xi_{\lambda}\right)\left(\begin{array}{c}
v \\
z
\end{array}\right),\left(\begin{array}{c}
v \\
z
\end{array}\right)\right\rangle \\
& =\int_{\mathbb{R}^{N}}|\nabla v|^{2}-\partial_{s} g\left(x, \varphi_{\lambda}\right) v^{2}-\lambda v^{2} d x+\int_{\mathbb{R}^{N}}|\nabla z|^{2}-\frac{g\left(x, \varphi_{\lambda}\right)}{\varphi_{\lambda}} z^{2}-\lambda z^{2} d x .
\end{aligned}
$$

Note that $\left(\begin{array}{c}v \\ z\end{array}\right) \in \operatorname{ker} D_{u u}^{2} G_{\lambda}\left(\xi_{\lambda}\right)$ if and only if for $v, z \in H^{1}\left(\mathbb{R}^{N}\right)$ and

$$
\mathfrak{I}\left(\Delta v+\partial_{s} g\left(x, \varphi_{\lambda}\right) v+\lambda v\right)=\mathfrak{I}\left(\Delta z+\frac{g\left(x, \varphi_{\lambda}\right)}{\varphi_{\lambda}} z+\lambda z\right)=0 .
$$

\subsection{The stability conditions}

Turning to the stability condition (SC) for a real solution $\xi_{\lambda}=\left(\begin{array}{c}\varphi_{\lambda} \\ 0\end{array}\right)$, we note that

$$
\begin{aligned}
& \left\{A \xi_{\lambda}, R^{-1} B \xi_{\lambda}\right\}^{\perp} \\
& =\left\{\left(\begin{array}{c}
v \\
z
\end{array}\right) \in X:\left\langle\left(\begin{array}{c}
v \\
z
\end{array}\right),\left(\begin{array}{c}
0 \\
\varphi_{\lambda}
\end{array}\right)\right\rangle_{X}=\left\langle\left(\begin{array}{c}
v \\
z
\end{array}\right), R^{-1} \mathfrak{I}\left(\begin{array}{c}
\varphi_{\lambda} \\
0
\end{array}\right)\right\rangle_{X}=0\right\} \\
& =\left\{\left(\begin{array}{c}
v \\
z
\end{array}\right) \in X:\left\langle z, \varphi_{\lambda}\right\rangle_{H^{1}}=\left(v, \varphi_{\lambda}\right)_{L^{2}}=0\right\} .
\end{aligned}
$$


Thus (SC) becomes: there exists $\delta>0$ such that

$$
\begin{aligned}
& \int_{\mathbb{R}^{N}}|\nabla v|^{2}-\partial_{s} g\left(x, \varphi_{\lambda}\right) v^{2}-\lambda v^{2} d x \geq \delta \int_{\mathbb{R}^{N}}|\nabla v|^{2}+v^{2} d x \\
& \text { for all } v \in H^{1}\left(\mathbb{R}^{N}\right) \text { with } \int_{\mathbb{R}^{N}} v \varphi_{\lambda} d x=0 \text {, and } \\
& \int_{\mathbb{R}^{N}}|\nabla z|^{2}-\frac{g\left(x, \varphi_{\lambda}\right)}{\varphi_{\lambda}} z^{2}-\lambda z^{2} d x \geq \delta \int_{\mathbb{R}^{N}}|\nabla z|^{2}+z^{2} d x \\
& \text { for all } z \in H^{1}\left(\mathbb{R}^{N}\right) \text { with } \int_{\mathbb{R}^{N}} \nabla z \cdot \nabla \varphi_{\lambda}+z \varphi_{\lambda} d x=0 .
\end{aligned}
$$

In this setting, the condition $(\mathrm{G})$ is satisfied if and only if there exist $\varepsilon, C>$ 0 such that, for all $v \in H^{1}\left(\mathbb{R}^{N}\right)$,

$$
\begin{aligned}
\int_{\mathbb{R}^{N}}|\nabla v|^{2}-\partial_{s} g\left(x, \varphi_{\lambda}\right) v^{2} d x & \geq \varepsilon \int_{\mathbb{R}^{N}}|\nabla v|^{2}+v^{2} d x-C \int_{\mathbb{R}^{N}} v^{2} d x, \\
\int_{\mathbb{R}^{N}}|\nabla v|^{2}-\frac{g\left(x, \varphi_{\lambda}\right)}{\varphi_{\lambda}} v^{2} d x & \geq \varepsilon \int_{\mathbb{R}^{N}}|\nabla v|^{2}+v^{2} d x-C \int_{\mathbb{R}^{N}} v^{2} d x .
\end{aligned}
$$

When $(\mathrm{G})$ is satisfied there are domains $D\left(L_{\lambda}^{1}\right)$ and $D\left(L_{\lambda}^{2}\right)$ in $H^{1}\left(\mathbb{R}^{N}\right)$ such that

$$
\begin{aligned}
& L_{\lambda}^{1} v=-\Delta v-\partial_{s} g\left(x, \varphi_{\lambda}\right) v-\lambda v \text { for } v \in D\left(L_{\lambda}^{1}\right) \text { and } \\
& L_{\lambda}^{2} v=-\Delta v-\frac{g\left(x, \varphi_{\lambda}\right)}{\varphi_{\lambda}} v-\lambda v \text { for } v \in D\left(L_{\lambda}^{2}\right)
\end{aligned}
$$

define self-adjoint operators in $L^{2}\left(\mathbb{R}^{N}\right)$. The self-adjoint operator $L_{\lambda}$ : $D\left(L_{\lambda}\right) \subset H \rightarrow H$ associated with the Hessian $D_{u u}^{2} G_{\lambda}\left(\left(\begin{array}{c}\varphi_{\lambda} \\ 0\end{array}\right)\right)$ is then given by $D\left(L_{\lambda}\right)=D\left(L_{\lambda}^{1}\right) \times D\left(L_{\lambda}^{2}\right)$ and

$$
L_{\lambda}\left(\begin{array}{c}
v \\
z
\end{array}\right)=\left(\begin{array}{c}
L_{\lambda}^{1} v \\
L_{\lambda}^{2} z
\end{array}\right) .
$$

Thus the stability condition $\left(\mathrm{SC}^{* *}\right)$ reduces to: there exists $\delta>0$ such that

$$
\begin{aligned}
& \int_{\mathbb{R}^{N}}|\nabla v|^{2}-\partial_{s} g\left(x, \varphi_{\lambda}\right) v^{2}-\lambda v^{2} d x \geq \delta \int_{\mathbb{R}^{N}} v^{2} d x \\
& \text { for all } v \in H^{1}\left(\mathbb{R}^{N}\right) \text { with } \int_{\mathbb{R}^{N}} v \varphi_{\lambda} d x=0, \text { and }
\end{aligned}
$$




$$
\begin{aligned}
& \int_{\mathbb{R}^{N}}|\nabla z|^{2}-\frac{g\left(x, \varphi_{\lambda}\right)}{\varphi_{\lambda}} z^{2}-\lambda z^{2} d x \geq \delta \int_{\mathbb{R}^{N}} z^{2} d x \\
& \text { for all } z \in H^{1}\left(\mathbb{R}^{N}\right) \text { with } \int_{\mathbb{R}^{N}} z \varphi_{\lambda} d x=0 .
\end{aligned}
$$

In other words, $L_{\lambda}^{1}$ and $L_{\lambda}^{2}$ are positive definite on the $L^{2}$-orthogonal complement of the stationary solution $\varphi_{\lambda}$.

Let us now consider the special situation covered by condition (B). Note that $L_{\lambda}: D\left(L_{\lambda}\right) \subset L^{2}\left(\mathbb{R}^{N}\right) \times L^{2}\left(\mathbb{R}^{N}\right) \rightarrow L^{2}\left(\mathbb{R}^{N}\right) \times L^{2}\left(\mathbb{R}^{N}\right)$ is a self-adjoint operator with

$$
\begin{aligned}
\sigma\left(L_{\lambda}\right) & =\sigma\left(L_{\lambda}^{1}\right) \cup \sigma\left(L_{\lambda}^{2}\right), \sigma_{e}\left(L_{\lambda}\right)=\sigma_{e}\left(L_{\lambda}^{1}\right) \cup \sigma_{e}\left(L_{\lambda}^{2}\right) \text { and } \\
M\left(L_{\lambda}\right) & =M\left(L_{\lambda}^{1}\right)+M\left(L_{\lambda}^{2}\right) .
\end{aligned}
$$

In particular,

$$
\inf \sigma_{e}\left(L_{\lambda}\right)>0 \Longleftrightarrow \inf \sigma_{e}\left(L_{\lambda}^{1}\right)>0 \text { and } \inf \sigma_{e}\left(L_{\lambda}^{2}\right)>0 .
$$

Finally, let us suppose that the real solution $\varphi_{\lambda}$ has the properties:

$$
\varphi_{\lambda}>0 \text { and } \inf \sigma_{e}\left(L_{\lambda}^{2}\right)>0 .
$$

(This is often the case for so-called ground-states.) Since $L_{\lambda}^{2} \varphi_{\lambda}=0$, this implies that 0 is an eigenvalue of $L_{\lambda}^{2}$ having a positive eigenfunction $\varphi_{\lambda}$. Hence $\inf \sigma\left(L_{\lambda}^{2}\right) \leq 0<\inf \sigma_{e}\left(L_{\lambda}^{2}\right)$. But, under mild hypotheses on $\frac{g\left(x, \varphi_{\lambda}\right)}{\varphi_{\lambda}}$, (see [29], for example, if $\frac{g\left(x, \varphi_{\lambda}\right)}{\varphi_{\lambda}} \in L^{\infty}\left(\mathbb{R}^{N}\right)$ ) this ensures that $\inf \sigma\left(L_{\lambda}^{2}\right)$ is a simple eigenvalue of $L_{\lambda}^{2}$ having a positive eigenfunction. Since eigenfunctions associated with different eigenvalues of $L_{\lambda}^{2}$ are $L^{2}$-orthogonal, we must have

$$
0=\inf \sigma\left(L_{\lambda}^{2}\right) \text { and } \operatorname{ker} L_{\lambda}^{2}=\operatorname{span}\left\{\varphi_{\lambda}\right\} .
$$

Then $M\left(L_{\lambda}\right)=M\left(L_{\lambda}^{1}\right)$ and, using Corollary 5.5, we see that the condition (B) holds provided that

$$
\inf \sigma_{e}\left(L_{\lambda}^{1}\right)>0, M\left(L_{\lambda}^{1}\right)=1 \text { and } \operatorname{ker} L_{\lambda}^{1}=\{0\} .
$$

When this is the case, stability is implied by the (VK) criterion which becomes simply

$$
\frac{d}{d \lambda} \int_{\mathbb{R}^{N}} \varphi_{\lambda}^{2} d x<0
$$

Remark 1. It should be emphasized that the condition (B) is never satisfied by an autonomous equation where $g$ is independent of $x: g(x, s)=g(s)$. This is because, from the stationary equation

$$
-\Delta \varphi_{\lambda}-g\left(\varphi_{\lambda}\right)=\lambda \varphi_{\lambda},
$$


it follows that any partial derivative $\partial_{j} \varphi_{\lambda}$ satisfies

$$
\left\{-\Delta-\partial_{s} g\left(\varphi_{\lambda}\right)-\lambda\right\} \partial_{j} \varphi_{\lambda}=0
$$

and so, if $\partial_{j} \varphi_{\lambda} \in H^{1}\left(\mathbb{R}^{N}\right), 0$ is also an eigenvalue of $\left\{-\Delta-\partial_{s} g\left(\varphi_{\lambda}\right)-\lambda\right\}$. Hence $\left\{A \xi_{\lambda}, \partial_{j} \xi_{\lambda}\right\} \subset \operatorname{ker} D_{u u}^{2} G_{\lambda}\left(\xi_{\lambda}\right)$. Even in the case where $\varphi_{\lambda}$ is a ground state obtained by constrained minimization as in Section 6 , it is known that the associated standing wave is not orbitally stable, see Remark 8.3.3 in [4]. This phenomenon occurs because the equation is now invariant under the action of the group of translations on $\mathbb{R}^{N}$ as well as the group $T(\theta)$. The results in Section 6 can be used to show that the standing wave associated with a ground state has the weaker stability property (WS). This was first established in [5]. See Theorem 8.6 (II) below for a generalization of this.

Remark 2. Standing waves of (CNLS) are non-stationary solutions of the form $T(\lambda t) \xi$. They are generated by solutions of (SNLS) with $\lambda \xi \neq 0$. Of course, the equivariance of the nonlinearity implies that $\xi=(\varphi, \psi)=(0,0)$ satisfies (SNLS) for all $\lambda \in \mathbb{R}$, but this generates the stationary solution $w(x, t) \equiv 0$. Recalling (7.2), the condition (4.4) for the stability of the solution $w \equiv 0$ becomes: there exist $\lambda \in \mathbb{R}$ and $\delta>0$ such that

$$
\int_{\mathbb{R}^{N}}|\nabla z|^{2}-f(x, 0) z^{2}-\lambda z^{2} d x \geq \delta \int_{\mathbb{R}^{N}}|\nabla z|^{2}+z^{2} d x
$$

for all $z \in H^{1}\left(\mathbb{R}^{N}\right)$. Note that this condition is clearly satisfied if $f(\cdot, 0) \in$ $L^{\infty}\left(\mathbb{R}^{N}\right)$.

\subsection{Summary}

Here is a list of the main points to be considered when trying to establish the orbital stability of standing waves of (CNLS).

(1) The nonlinearity $g(x, s)=f\left(x, s^{2}\right) s$ should be such that $\Phi \in$ $C^{2}\left(H^{1}\left(\mathbb{R}^{N}\right)\right)$ where

$$
\Phi(u)=\int_{\mathbb{R}^{N}} F\left(x, u(x)^{2}\right) d x \text { and } F(x, s)=\int_{0}^{s} f(x, \tau) d \tau .
$$

(2) In most cases one deals with standing waves $T(\lambda t) \xi_{\lambda}$ where $\xi_{\lambda}$ is a "real" solution of the stationary equation, that is

$\xi_{\lambda}=\left(\begin{array}{c}\varphi_{\lambda} \\ 0\end{array}\right)$ where $\varphi_{\lambda} \in\{0\}$ and $-\Delta \varphi_{\lambda}-g\left(x, \varphi_{\lambda}\right)=\lambda \varphi_{\lambda}$ for some $\lambda \neq 0$,

$T(\lambda t) \xi_{\lambda}=\left(\begin{array}{c}\varphi_{\lambda} \cos \lambda t \\ -\varphi_{\lambda} \sin \lambda t\end{array}\right)$

and, in complex notation, the standing wave is then $e^{-i \lambda t} \varphi_{\lambda}(x)$. 
See Corollary 2.3 of [30] and Proposition 5.2 of [31] for a discussion of the place of real solutions with respect to other solutions of (SNLS).

(3) The hypotheses on the nonlinearity should ensure that (GWP at $\xi_{\lambda}$ ) holds and that there exist $\varepsilon, C>0$ such that, for all $v \in H^{1}\left(\mathbb{R}^{N}\right)$,

$$
\begin{aligned}
\int_{\mathbb{R}^{N}}|\nabla v|^{2}-\partial_{s} g\left(x, \varphi_{\lambda}\right) v^{2} d x & \geq \varepsilon \int_{\mathbb{R}^{N}}|\nabla v|^{2} d x-C \int_{\mathbb{R}^{N}} v^{2} d x, \\
\int_{\mathbb{R}^{N}}|\nabla v|^{2}-\frac{g\left(x, \varphi_{\lambda}\right)}{\varphi_{\lambda}} v^{2} d x & \geq \varepsilon \int_{\mathbb{R}^{N}}|\nabla v|^{2} d x-C \int_{\mathbb{R}^{N}} v^{2} d x .
\end{aligned}
$$

Then condition $(\mathrm{G})$ is satisfied and we can consider the self-adjoint operators that are uniquely defined by

$$
\begin{aligned}
L_{\lambda}^{1}: D\left(L_{\lambda}^{1}\right) \subset L^{2}\left(\mathbb{R}^{N}\right) \rightarrow L^{2}\left(\mathbb{R}^{N}\right) \text { where } D\left(L_{\lambda}^{1}\right) \subset H^{1}\left(\mathbb{R}^{N}\right) \text { and } \\
L_{\lambda}^{1} v=-\Delta v-\partial_{s} g\left(x, \varphi_{\lambda}\right) v-\lambda v \text { for } v \in D\left(L_{\lambda}^{1}\right), \\
L_{\lambda}^{2}: D\left(L_{\lambda}^{2}\right) \subset L^{2}\left(\mathbb{R}^{N}\right) \rightarrow L^{2}\left(\mathbb{R}^{N}\right) \text { where } D\left(L_{\lambda}^{2}\right) \subset H^{1}\left(\mathbb{R}^{N}\right) \\
L_{\lambda}^{2} v=-\Delta v-\frac{g\left(x, \varphi_{\lambda}\right)}{\varphi_{\lambda}} v-\lambda v \text { for } v \in D\left(L_{\lambda}^{2}\right) .
\end{aligned}
$$

(4) A sufficient condition for stability is given by $\left(\mathrm{SC}^{* *}\right)$ : there exists $\delta>0$ such that

$$
\begin{aligned}
& \int_{\mathbb{R}^{N}}|\nabla v|^{2}-\partial_{s} g\left(x, \varphi_{\lambda}\right) v^{2}-\lambda v^{2} d x \geq \delta \int_{\mathbb{R}^{N}} v^{2} d x \\
& \text { for all } v \in H^{1}\left(\mathbb{R}^{N}\right) \text { with } \int_{\mathbb{R}^{N}} v \varphi_{\lambda} d x=0, \text { and } \\
& \int_{\mathbb{R}^{N}}|\nabla z|^{2}-\frac{g\left(x, \varphi_{\lambda}\right)}{\varphi_{\lambda}} z^{2}-\lambda z^{2} d x \geq \delta \int_{\mathbb{R}^{N}} z^{2} d x \\
& \text { for all } z \in H^{1}\left(\mathbb{R}^{N}\right) \text { with } \int_{\mathbb{R}^{N}} z \varphi_{\lambda} d x=0 .
\end{aligned}
$$

Alternatively, one can try the approach based on the condition (VK).

(5) For this the hypotheses on $f$ (equivalently, $g$ ) should ensure that

$$
\begin{aligned}
& \inf \sigma_{e}\left(L_{\lambda}^{1}\right)>0, M\left(L_{\lambda}^{1}\right)=1 \text { and } \operatorname{ker} L_{\lambda}^{1}=\{0\}, \\
& \inf \sigma_{e}\left(L_{\lambda}^{2}\right)>0,0=\inf \sigma\left(L_{\lambda}^{2}\right) \text { and } \operatorname{ker} L_{\lambda}^{2}=\operatorname{span}\left\{\varphi_{\lambda}\right\} .
\end{aligned}
$$

Then the condition (B) is satisfied and $\varphi_{\lambda}$ is embedded in a $C^{1}$-curve of stationary solutions. The standing wave is orbitally stable provided that

$$
\frac{d}{d \lambda} \int_{\mathbb{R}^{N}} \varphi_{\lambda}^{2} d x<0
$$




\section{NLS with a power-law nonlinearity}

In the previous section we discussed the procedure for analyzing orbital stability of standing waves in the context of (CNLS). Now we exhibit some of the results that have been obtained by implementing this approach in the most common case where the nonlinearity is a power of the amplitude. We consider (CNLS) with

$$
f\left(x,|w|^{2}\right)=P(x)+V(x)|w|^{p-1}
$$

where

(C1) $1<p<2^{*}-1$ and $P, V \in L^{\infty}\left(\mathbb{R}^{N}, \mathbb{R}\right)$ where $2^{*}=\infty$ for $N=1$ and 2 and $2^{*}=2 N /(N-2)$ for $N \geq 3$. Thus, $1<p<\infty$ for $N=1,2$ and $1<p<1+\frac{4}{N-2}$ for $N \geq 3$.

In general, $V$ can change sign but when $V \geq 0$ the nonlinearity is said to be self-focusing whereas when $V \leq 0$ it is defocusing.

Under these assumptions, we have that, for $x \in \mathbb{R}^{N}$ and $s \geq 0$,

$$
\begin{aligned}
f(x, s) & =P(x)+V(x) s^{\frac{p-1}{2}}, \\
F(x, s) & =\int_{0}^{s} f(x, \tau) d \tau=P(x) s+\frac{2 V(x)}{p+1} s^{\frac{p+1}{2}} \\
\text { and } \Phi(u) & =\int_{\mathbb{R}^{N}} F\left(x, u(x)^{2}\right) d x=\int_{\mathbb{R}^{N}} P(x) u(x)^{2}+\frac{2 V(x)}{p+1} u(x)^{p+1} d x .
\end{aligned}
$$

Since $P, V \in L^{\infty}\left(\mathbb{R}^{N}\right)$ and $2<p+1<2^{*}$ it is well-known that $\Phi \in C^{2}\left(H^{1}\left(\mathbb{R}^{N}\right), \mathbb{R}\right)$ with

$$
\Phi^{\prime}(u) v=\int_{\mathbb{R}^{N}} f\left(x, u(x)^{2}\right) u(x) v(x) d x \text { for all } u, v \in H^{1}\left(\mathbb{R}^{N}\right) .
$$

Furthermore, the expression $L u=-\Delta u-P u$ defines a self-adjoint operator $L: H^{2}\left(\mathbb{R}^{N}\right) \subset L^{2}\left(\mathbb{R}^{N}\right) \rightarrow L^{2}\left(\mathbb{R}^{N}\right)$ which is bounded below.

Remark. By Corollary 6.1.5 of [4], the condition (C1) ensures that (GWP at 0 ) holds. Since $f(\cdot, 0)=P \in L^{\infty}\left(\mathbb{R}^{N}\right)$, it follows from (7.3) and Theorem 4.5 that the stationary solution $w \equiv 0$ is stable. Theorems 8.1 and 8.5 below establish the existence of a branch $\lambda \mapsto \varphi_{\lambda} \in H^{1}\left(\mathbb{R}^{N}\right) \backslash\{0\}$ of solutions of (RSNLS) and conditions are given ensuring the orbital stability of the associated standing waves $w_{\lambda}(x, t)=e^{i \lambda t} \varphi_{\lambda}(x)$. In both cases, the stable solutions bifurcate from the stationary solution $w \equiv 0$ in a way that is reminiscent of the Lyapunov Centre Theorem, [1]. In Theorem 8.1, $\left\|\varphi_{\lambda}\right\|_{H^{1}} \rightarrow 0$ as the frequency $\lambda \rightarrow \Lambda$ where $\Lambda$ is a simple eigenvalue of the linear operator $L=-\Delta+P$. In Theorem 8.5, $P$ is constant and $L$ has no 
eigenvalues. In that case, $\left\|\varphi_{\lambda}\right\|_{H^{1}} \rightarrow 0$ as the frequency $\lambda \rightarrow P$ from below and we have bifurcation from the bottom of the essential spectrum of $L$.

\subsection{Bifurcation from an eigenvalue}

First we deal with the case where the potential $P$ is such that

(C2) $\Lambda<\inf \sigma_{e}(L)$ where $\Lambda=\inf \sigma(L)$.

Under the assumptions $(\mathrm{C} 1)$ and $(\mathrm{C} 2), \Lambda$ is a simple eigenvalue of $L$ with a strictly positive eigenfunction $\phi \in H^{2}\left(\mathbb{R}^{N}\right) \cap C^{1}\left(\mathbb{R}^{N}\right)$ which we normalize so that

$$
\int_{\mathbb{R}^{N}} \phi^{2} d x=1
$$

(See Theorem 3.20 in [29], for example.) The condition (C2) implies that $P$ is not constant on $\mathbb{R}^{N}$. It is easy to formulate explicit properties of $P$ that imply $(\mathrm{C} 2)$.

Various conditions ensuring that (CNLS) is globally well-posed are established in Section 6.1 of [4] and from them we obtain the following information. Under the hypothesis (C1), the property (GWP at $\xi$ ) holds for all $\xi \in H^{1}\left(\mathbb{R}^{N}\right) \times H^{1}\left(\mathbb{R}^{N}\right)$ provided that $1<p<1+\frac{4}{N}$. Furthermore, for $1+\frac{4}{N} \leq p<1+\frac{4}{N-2}$, there exists a constant $C>0$ such that (GWP at $\xi$ ) holds for all $\xi \in H^{1}\left(\mathbb{R}^{N}\right) \times H^{1}\left(\mathbb{R}^{N}\right)$ with $\|\xi\|_{L^{2}}<C$. If, in addition to $(\mathrm{C} 1), V \leq 0$ on $\mathbb{R}^{N}$ then (GWP at $\xi$ ) holds for all $\xi \in H^{1}\left(\mathbb{R}^{N}\right) \times H^{1}\left(\mathbb{R}^{N}\right)$ for $1<p<1+\frac{4}{N-2}$.

Theorem 8.1. Suppose that $(\mathrm{C} 1)$ and $(\mathrm{C} 2)$ are satisfied.

(I) If $\int_{\mathbb{R}^{N}} V \phi^{p+1} d x>0$, there exist $\delta>0$ and $\varphi \in C^{1}\left((\Lambda-\delta, \Lambda), H^{2}\left(\mathbb{R}^{N}\right)\right)$ having the following properties for all $\lambda \in(\Lambda-\delta, \Lambda)$,

$$
\begin{aligned}
& \varphi(\lambda)=\varphi_{\lambda} \in C\left(\mathbb{R}^{N}\right) \cap L^{\infty}\left(\mathbb{R}^{N}\right), \quad \varphi_{\lambda}>0, \\
& \text { there exists } \mu(\lambda)>0 \text { such that } \lim _{|x| \rightarrow 0} e^{\mu(\lambda)|x|} \varphi_{\lambda}(x)=0, \\
& \quad \lim _{\lambda \rightarrow \Lambda}\left\|\varphi_{\lambda}\right\|_{H^{2}\left(\mathbb{R}^{N}\right)}=\lim _{\lambda \rightarrow \Lambda}\left\|\varphi_{\lambda}\right\|_{L^{\infty}\left(\mathbb{R}^{N}\right)}=0, \\
& \Delta \varphi_{\lambda}(x)+f\left(x, \varphi_{\lambda}(x)^{2}\right) \varphi_{\lambda}(x)+\lambda \varphi_{\lambda}(x)=0 \text { for all } x \in \mathbb{R}^{N} .
\end{aligned}
$$

(II) If $\int_{\mathbb{R}^{N}} V \phi^{p+1} d x<0$, there exist $\delta>0$ and $\varphi \in C^{1}\left((\Lambda, \Lambda+\delta), H^{2}\left(\mathbb{R}^{N}\right)\right)$ having the same properties for all $\lambda \in(\Lambda, \Lambda+\delta)$.

In both cases, we have the expansion

$$
\varphi_{\lambda}=\left[\frac{\Lambda-\lambda}{\int_{\mathbb{R}^{N}} V \phi^{p+1} d x}\right]^{1 /(p-1)}\{\phi+v(\lambda)\}
$$


where $\phi$ is normalized so that $\int_{\mathbb{R}^{N}} \phi^{2} d x=1$ and $\|v(\lambda)\|_{H^{2}} \rightarrow 0$ as $\lambda \rightarrow \Lambda$.

In case (I) the standing wave associated with $\varphi_{\lambda}$ is orbitally stable if $1<p<2^{*}-1$. If $V \leq 0$ on $\mathbb{R}^{N}$ and $V<0$ on a set of positive measure, the solutions in case (II) are also orbitally stable if $1<p<2^{*}-1$.

Remark 1. The situation where $V \equiv 1$ and $\lim _{|x| \rightarrow \infty} P(x)=0$ was dealt with in [27]. Theorem 8.1 is a local result and it should be understood in the sense that, by choosing a sufficiently small value of $\delta>0$ all the conclusions are valid. However the factors influencing the choice of $\delta$ are not the same in all cases. To obtain a branch of stable solutions in case (I), it must be small enough so that (i) a smooth branch exists for $\delta \in(\Lambda-\delta, \Lambda)$, (ii) $\frac{d}{d \lambda}\left\|\varphi_{\lambda}\right\|_{L^{2}}<0$ on $(\Lambda-\delta, \Lambda)$ and (iii) $\left\|\varphi_{\lambda}\right\|_{L^{2}}$ is sufficiently small that there is global existence of the initial value problem for (CNLS) for all initial conditions sufficiently close to $\varphi_{\lambda}$. In the defocusing case where $V \leq 0$ on $\mathbb{R}^{N}$, only point (i) needs to be ensured since there is global existence for all initial conditions and stability is not derived from the VK-condition. This difference is of importance when considering global extensions of the branch.

Remark 2. The first part of the following proof establishes the existence of a branch $\lambda \mapsto \varphi_{\lambda}$ through bifurcation from a simple eigenvalue at $\Lambda$. The bifurcation is to the left if $\int_{\mathbb{R}^{N}} V \phi^{p+1} d x>0$ and to the right if $\int_{\mathbb{R}^{N}} V \phi^{p+1} d x<0$. In the first case, $\varphi_{\lambda}$ turns out to be a saddle point of the augmented Hamiltonian $G_{\lambda}$, whereas in the second case it is a local minimum. This difference influences the proof of orbital stability. For (CNLS) the condition (VK) is $\frac{d}{d \lambda} \int_{\mathbb{R}^{N}} \varphi_{\lambda}^{2} d x<0$ and this occurs only in case (I) where the condition (B) is also satisfied. Hence in this case stability follows from Proposition 5.2. In case (II), $\frac{d}{d \lambda} \int_{\mathbb{R}^{N}} \varphi_{\lambda}^{2} d x<0$ and (B) is not satisfied. However, in this case it is easy to check ( $\left.\mathrm{SC}^{* *}\right)$ directly and so stability follows from Corollary 3.4.

Proof. Bifurcation Let $2^{* *}=\infty$ for $N \leq 4$ and $2^{* *}=2 N /(N-4)$ for $N \geq 5$. Then $H^{2}\left(\mathbb{R}^{N}\right)$ is continuously embedded in $L^{q}\left(\mathbb{R}^{N}\right)$ for $2 \leq q<2^{* *}$ and so $(\mathrm{C} 1)$ ensures that $u \mapsto f\left(x, u^{2}\right) u$ maps $H^{2}\left(\mathbb{R}^{N}\right)$ continuously into $L^{2}\left(\mathbb{R}^{N}\right)$. Indeed, the hypothesis $(\mathrm{C} 1)$ ensures that we can define a function $R \in C^{1}\left(\mathbb{R} \times H^{2}\left(\mathbb{R}^{N}\right), L^{2}\left(\mathbb{R}^{N}\right)\right)$ by

$$
\begin{aligned}
R(\lambda, u) & =\Delta u+f\left(x, u^{2}\right) u+\lambda u \\
& =\Delta u+P u+V(x)|u|^{p-1} u+\lambda u .
\end{aligned}
$$


Furthermore, $D_{u} R(\lambda, 0)=\Delta+P+\lambda: H^{2}\left(\mathbb{R}^{N}\right) \rightarrow L^{2}\left(\mathbb{R}^{N}\right)$ is a Fredholm operator of index zero for all $\lambda<\inf \sigma_{e}(L)$. Also

$$
\text { ker } D_{u} R(\Lambda, 0)=\operatorname{span}\{\phi\} \text { and } r g e D_{u} R(\Lambda, 0)=W
$$

where $W=\left\{u \in L^{2}\left(\mathbb{R}^{N}\right): \int_{\mathbb{R}^{N}} u \phi d x=0\right\}$. Since $D_{\lambda} D_{u} R(\Lambda, 0) \phi=\phi$, it follows that

$$
L^{2}\left(\mathbb{R}^{N}\right)=\operatorname{rge} D_{u} R(\Lambda, 0) \oplus \operatorname{span}\left\{D_{\lambda} D_{u} R(\Lambda, 0) \phi\right\}
$$

and then, by the fundamental theorem of Crandall and Rabinowitz on bifurcation from a simple eigenvalue, Theorem 1.7 in [7], there exist $\delta>0$, $\mu \in C((-\delta, \delta), \mathbb{R})$ and $z \in C\left((-\delta, \delta), H^{2}\left(\mathbb{R}^{N}\right)\right)$ such that

$$
\begin{aligned}
& \mu(0)=\Lambda, z(0)=0, R(\mu(s), s[\phi+z(s)])=0 \text { and } \\
& z(s) \in Z=\left\{u \in H^{2}\left(\mathbb{R}^{N}\right): \int_{\mathbb{R}^{N}} u \phi d x=0\right\}=W \cap H^{2}\left(\mathbb{R}^{N}\right)
\end{aligned}
$$

for all $s \in(-\delta, \delta)$. Furthermore, there is an open neighbourhood $V$ of $(\Lambda, 0)$ in $\mathbb{R} \times H^{2}\left(\mathbb{R}^{N}\right)$ such that if $(\lambda, u) \in V$ and $R(\lambda, u)=0$ then either $u=0$ or $(\lambda, u)=(\mu(s), s[\phi+z(s)])$ for some $s \in(-\delta, \delta)$. Noting that $R(\lambda, u)$ is an odd function of $u$, we have that $R(\mu(s),-s[\phi+z(s)])=0$ for $s$ near zero and so there exists $s^{\prime} \in(-\delta, \delta)$ such that

$$
(\mu(s),-s[\phi+z(s)])=\left(\mu\left(s^{\prime}\right), s^{\prime}\left[\phi+z\left(s^{\prime}\right)\right]\right) .
$$

By the orthogonality of $\phi$ and $z(s)$ and $\phi$ and $z\left(s^{\prime}\right)$, this implies that $s^{\prime}=-s$ and then $\mu(-s)=\mu(s)$ and $z(-s)=z(s)$. Henceforth we only need to discuss $s \in(0, \delta)$.

Setting $u_{s}=s[\phi+z(s)]$, standard regularity theory (see for example, Theorem 2.17 of [29]) shows that $u_{s} \in C\left(\mathbb{R}^{N}\right) \cap L^{\infty}\left(\mathbb{R}^{N}\right), \lim _{|x| \rightarrow \infty} u_{s}(x)=0$ and that $\left\|u_{s}\right\|_{L^{\infty}} \rightarrow 0$ as $s \rightarrow 0$. It follows that

$$
P+V\left|u_{s}\right|^{p-1} \in L^{\infty}\left(\mathbb{R}^{N}\right) .
$$

Then defining $L_{s}$ by

$$
L_{s}=-\Delta-P-V\left|u_{s}\right|^{p-1},
$$

we have that $L_{s}: H^{2}\left(\mathbb{R}^{N}\right) \subset L^{2}\left(\mathbb{R}^{N}\right) \rightarrow L^{2}\left(\mathbb{R}^{N}\right)$ is self-adjoint and $L_{s} \rightarrow L_{0}=L$ in $B\left(H^{2}\left(\mathbb{R}^{N}\right), L^{2}\left(\mathbb{R}^{N}\right)\right)$. Since $\lim _{|x| \rightarrow \infty} V(x)\left|u_{s}(x)\right|^{p-1}=0$, $\inf \sigma_{e}\left(L_{s}\right)=\inf \sigma_{e}(L)$ for all $s \in(-\delta, \delta)$. Furthermore $L_{s} u_{s}=\mu(s) u_{s}$ and $\mu(s) \rightarrow \Lambda=\inf \sigma(L)<\inf \sigma_{e}(L)$. It follows that, for $\delta>0$ small enough, $\mu(s)=\inf \sigma\left(L_{s}\right)<\inf \sigma_{e}\left(L_{s}\right)$ and consequently we have that $u_{s}$ does not 
change sign on $\mathbb{R}^{N}$ and decays exponentially to zero as $|x| \rightarrow \infty$. See Theorem 3.20 in [29], for example. Since $s>0$ and $\phi>0$, this implies that $u_{s}>0$ on $\mathbb{R}^{N}$.

From the fact that $P, V$ and $u_{s} \in L^{\infty}\left(\mathbb{R}^{N}\right)$, it follows immediately from point (3) in the summary at the end of Section 7 that condition $(G)$ is satisfied.

We now show $\mu \in C^{1}((0, \delta), \mathbb{R})$ and $z \in C^{1}\left((0, \delta), H^{2}\left(\mathbb{R}^{N}\right)\right)$. The equation $R(\lambda, u)=0$ can be written as

$$
L u-\lambda u-M(u)=0
$$

where $M(u)=V|u|^{p-1} u$ and $L-\Lambda: Z \rightarrow W$ is an isomorphism.

Consider $\Gamma:(0, \delta) \times \mathbb{R} \times Z \rightarrow L^{2}\left(\mathbb{R}^{N}\right)$ defined by

$$
\Gamma(s, \mu, z)=L(\phi+z)-s^{p-1} M(\phi+z)-\mu(\phi+z) .
$$

The hypothesis $(\mathrm{C} 1)$ ensures that $\Gamma \in C^{1}\left((0, \delta) \times \mathbb{R} \times Z, L^{2}\left(\mathbb{R}^{N}\right)\right)$ and, for all $s \in(0, \delta)$,

$$
D_{(\mu, z)} \Gamma(s, \Lambda, 0)(\nu, w)=-\nu \phi+L w-s^{p-1} M^{\prime}(\phi) w-\Lambda w
$$

We know that $S(\nu, w)=-\nu \phi+L w-\Lambda w$ is an isomorphism from $\mathbb{R} \times Z$ onto $L^{2}\left(\mathbb{R}^{N}\right)$. Since $D_{(\mu, z)} \Gamma(s, \mu(s), z(s)) \rightarrow S$ as $s \rightarrow 0$, it follows that $D_{(\mu, z)} \Gamma(s, \mu(s), z(s)): \mathbb{R} \times Z \rightarrow L^{2}\left(\mathbb{R}^{N}\right)$ is also an isomorphism provided that $\delta>0$ is made small enough. But $\Gamma(s, \mu(s), z(s))=0$ and so the implicit function theorem implies that $\mu \in C^{1}((0, \delta), \mathbb{R})$ and $z \in C^{1}((0, \delta), Z)$ with

$$
\begin{aligned}
0 & =\frac{d}{d s} \Gamma(s, \mu(s), z(s))=L z^{\prime}(s)-(p-1) s^{p-2} M(\phi+z(s)) \\
& -s^{p-1} M^{\prime}(\phi+z(s)) z^{\prime}(s)-\mu^{\prime}(s)(\phi+z(s))-\mu(s) z^{\prime}(s)
\end{aligned}
$$

Hence

$$
\mu^{\prime}(s)=-s^{p-2}\left((p-1) M(\phi+z(s))+M^{\prime}(\phi+z(s)) s z^{\prime}(s), \phi\right)
$$

where in a slight abuse of notation, we are now using $(\cdot, \cdot)$ to denote the usual scalar product on $L^{2}\left(\mathbb{R}^{N}\right)$. Since $z \in C\left((-\delta, \delta), H^{2}\left(\mathbb{R}^{N}\right)\right)$ and $M \in C^{1}\left(H^{2}\left(\mathbb{R}^{N}\right), L^{2}\left(\mathbb{R}^{N}\right)\right)$, this implies that there are constants $A$ and $B$ such that

$$
s\left|\mu^{\prime}(s)\right| \leq A s^{p-1}+B s\left\|s z^{\prime}(s)\right\|_{H^{2}} .
$$


But

$$
\begin{aligned}
& (L-\Lambda) z^{\prime}(s) \\
& =(p-1) s^{p-2} M(\phi+z)+s^{p-1} M^{\prime}(\phi+z) z^{\prime}(s)+\mu^{\prime}(s)(\phi+z) \\
& \quad+[\mu(s)-\Lambda] z^{\prime}(s)
\end{aligned}
$$

so there are constants $C, D, E$ such that

$$
\begin{aligned}
& \left\|(L-\Lambda) s z^{\prime}(s)\right\|_{L^{2}} \\
& \leq s^{p-1} C+s^{p-1} D\left\|s z^{\prime}(s)\right\|_{H^{2}}+E s\left|\mu^{\prime}(s)\right|+|\mu(s)-\Lambda|\left\|s z^{\prime}(s)\right\|_{L^{2}} \\
& \leq s^{p-1} C+s^{p-1} D\left\|s z^{\prime}(s)\right\|_{H^{2}}+E\left\{A s^{p-1}+B s\left\|s z^{\prime}(s)\right\|_{H^{2}}\right\} \\
& \quad+|\mu(s)-\Lambda|\left\|s z^{\prime}(s)\right\|_{L^{2}}
\end{aligned}
$$

Since $L-\Lambda: Z \rightarrow W$ is an isomorphism, there exists $\varepsilon>0$ such that

$$
\|(L-\Lambda) z\|_{L^{2}} \geq \varepsilon\|z\|_{H^{2}} \text { for all } z \in Z \text {. }
$$

Hence

$\varepsilon\left\|s z^{\prime}(s)\right\|_{H^{2}} \leq s^{p-1} C_{1}+\left\{s^{p-1} D_{1}+E_{1} s\right\}\left\|s z^{\prime}(s)\right\|_{H^{2}}+|\mu(s)-\Lambda|\left\|s z^{\prime}(s)\right\|_{H^{2}}$ from which it follows that

$$
\left\|s z^{\prime}(s)\right\|_{H^{2}} \rightarrow 0 \text { as } s \rightarrow 0, \text { since } \mu(s) \rightarrow \Lambda .
$$

We now have that

$$
\begin{aligned}
\frac{\mu^{\prime}(s)}{s^{p-2}} & =-\left((p-1) M(\phi+z(s))+M^{\prime}(\phi+z(s)) s z^{\prime}(s), \phi\right) \\
& \rightarrow-(p-1)(M(\phi), \phi) \text { as } s \rightarrow 0 .
\end{aligned}
$$

If $(M(\phi), \phi) \neq 0$ we can chose $\delta>0$ so that $\mu^{\prime}(s) \neq 0$ for all $s \in(0, \delta)$ and then set $\varphi(\lambda)=s[\phi+z(s)]$ where $\lambda=\mu(s)$. Now $\varphi \in C^{1}\left(J, H^{2}\left(\mathbb{R}^{N}\right)\right)$ where $J$ is the interval $\{\mu(s): 0<s<\delta\}$.

To complete the discussion of the asymptotic behaviour of the branch as $\lambda=\mu(s) \rightarrow \Lambda$, we note that

$$
\begin{aligned}
& \frac{\Lambda-\mu(s)}{(M(\phi), \phi)}>0 \text { for all } s \in(0, \delta) \text { and } \\
& \lim _{s \rightarrow 0} \frac{\mu(s)-\Lambda}{s^{p-1}}=\lim _{s \rightarrow 0} \frac{\mu^{\prime}(s)}{(p-1) s^{p-2}}=-(M(\phi), \phi), \text { by L'Hospital's rule. }
\end{aligned}
$$

Hence

$$
\lim _{s \rightarrow 0}\left\{\frac{\Lambda-\mu(s)}{(M(\phi), \phi)}\right\} \frac{1}{s^{p-1}}=1 \text { and so } \lim _{s \rightarrow 0} \frac{s}{\left\{\frac{\Lambda-\mu(s)}{(M(\phi), \phi)}\right\}^{\frac{1}{p-1}}}=1
$$


showing that

$$
s=\left\{\frac{\Lambda-\mu(s)}{(M(\phi), \phi)}\right\}^{\frac{1}{p-1}}\{1+\rho(s)\} \text { where } \rho(s) \rightarrow 0 \text { as } s \rightarrow 0 .
$$

Thus

$$
\varphi(\lambda)=s[\phi+z(s)]=\left\{\frac{\Lambda-\mu(s)}{(M(\phi), \phi)}\right\}^{\frac{1}{p-1}}\{\phi+w(s)\}
$$

where $w(s)=z(s)+\rho(s)\{\phi+z(s)\} \rightarrow 0$ in $H^{2}\left(\mathbb{R}^{N}\right)$ as $s \rightarrow 0$.

At this point we have proved the statements (I) and (II).

Orbital stability Turning to the stability of the standing wave generated by $\varphi_{\lambda}$, we know from point (5) of the summary at the end of Section 7 that the condition (VK) amounts to showing that $\frac{d}{d \lambda} \int_{\mathbb{R}^{N}} \varphi_{\lambda}^{2} d x<0$. But from the asymptotic behaviour of the branch, we have that

$$
\frac{d}{d \lambda}\left\|\varphi_{\lambda}\right\|_{L^{2}}=2\left(\varphi_{\lambda}, \frac{d}{d s} \varphi_{\lambda}\right) \frac{d s}{d \lambda}=2 s\left(\phi+z(s), \phi+z(s)+s z^{\prime}(s)\right) \frac{1}{\mu^{\prime}(s)}
$$

where

$$
\left(\phi+z(s), \phi+z(s)+s z^{\prime}(s)\right) \rightarrow(\phi, \phi) \text { as } s \rightarrow 0 .
$$

Then, for all $s \in(0, \delta)$ with $\delta$ small enough, we have the

$$
\frac{d}{d \lambda}\left\|\varphi_{\lambda}\right\|_{L^{2}} \text { has the same sign as }-(M(\phi), \phi) \text {. }
$$

By choosing $\delta>0$ small enough, it follows from Corollary 6.1 .5 of [4] that we can assume that (GWP at $\left.\xi_{\lambda}=\left(\varphi_{\lambda}, 0\right)\right)$ holds for all $\lambda$ near $\Lambda$. To discuss the stability of the standing wave $T(\lambda t) \xi_{\lambda} \approx e^{-i \lambda t} \varphi_{\lambda}$ we need to consider the Hessian $D_{u u}^{2} G_{\lambda}\left(\xi_{\lambda}\right)$ and this involves the following self-adjoint operators.

$$
\begin{aligned}
L_{\lambda}^{1} & : D\left(L_{\lambda}^{1}\right) \subset L^{2}\left(\mathbb{R}^{N}\right) \rightarrow L^{2}\left(\mathbb{R}^{N}\right) \text { where } D\left(L_{\lambda}^{1}\right) \subset H^{1}\left(\mathbb{R}^{N}\right) \\
L_{\lambda}^{1} v & =-\Delta v-\partial_{s} g\left(x, \varphi_{\lambda}\right) v-\lambda v \text { for } v \in D\left(L_{\lambda}^{1}\right) \\
& =-\Delta v-P v-p V \varphi_{\lambda}^{p-1} v-\lambda v, \text { and } \\
L_{\lambda}^{2} & : D\left(L_{\lambda}^{2}\right) \subset L^{2}\left(\mathbb{R}^{N}\right) \rightarrow L^{2}\left(\mathbb{R}^{N}\right) \text { where } D\left(L_{\lambda}^{2}\right) \subset H^{1}\left(\mathbb{R}^{N}\right) \\
L_{\lambda}^{2} v & =-\Delta v-\frac{g\left(x, \varphi_{\lambda}\right)}{\varphi_{\lambda}} v-\lambda v \text { for } v \in D\left(L_{\lambda}^{2}\right) \\
& =-\Delta v-P v-V \varphi_{\lambda}^{p-1} v-\lambda v .
\end{aligned}
$$

We have $\lambda=\mu(s)$ and $\varphi_{\lambda}=u_{s}$ for some $s \in(0, \delta)$ and so, in the earlier notation $L_{\lambda}^{2}=L_{s}-\mu(s)$. Furthermore, since $P, V$ and $\varphi_{\lambda} \in L^{\infty}\left(\mathbb{R}^{N}\right)$, $D\left(L_{\lambda}^{1}\right)=D\left(L_{\lambda}^{2}\right)=H^{2}\left(\mathbb{R}^{N}\right)$ We have already shown that $\mu(s)=\inf \sigma\left(L_{s}\right)$ 
and so $\inf \sigma\left(L_{\lambda}^{2}\right)=0$ with $\operatorname{ker} L_{\lambda}^{2}=\operatorname{ker}\left[L_{s}-\mu(s)\right]=\operatorname{span}\left\{u_{s}\right\}=\operatorname{span}\left\{\varphi_{\lambda}\right\}$. Also $\inf \sigma_{e}\left(L_{\lambda}^{2}\right)=\inf \sigma_{e}\left(L_{s}\right)-\mu(s)=\inf \sigma_{e}(L)-\mu(s)>0$ since $\mu(s) \rightarrow$ $\Lambda<\inf \sigma_{e}(L)$. Thus we have that

$$
\left(L_{\lambda}^{2} v, v\right) \geq \delta_{1}(v, v) \text { for all } v \in H^{1}\left(\mathbb{R}^{N}\right) \text { with }\left(v, \varphi_{\lambda}\right)=0 .
$$

As for $L_{\lambda}^{2}, \inf \sigma_{e}\left(L_{\lambda}^{1}\right)>0$ for $\lambda$ near $\Lambda$. However, as we now show, $\left.M\left(L_{\lambda}^{1}\right)\right)=$ 1 in case (I) and $\left.M\left(L_{\lambda}^{1}\right)\right)=0$ in case (II).

(I) Case $(M(\phi), \phi)>0$.

Concerning the spectrum of $L_{\lambda}^{1}$ we have that

$$
0=\left(L_{\lambda}^{2} \varphi_{\lambda}, \varphi_{\lambda}\right)=\left(L_{\lambda}^{1} \varphi_{\lambda}, \varphi_{\lambda}\right)+(p-1)\left(M\left(\varphi_{\lambda}\right), \varphi_{\lambda}\right)
$$

so that

$$
\left(L_{\lambda}^{1} \varphi_{\lambda}, \varphi_{\lambda}\right) \leq-(p-1)\left(M\left(\varphi_{\lambda}\right), \varphi_{\lambda}\right)=-(p-1) s^{p+1}(M(\phi+z(s)), \phi+z(s))
$$

where

$$
(M(\phi+z(s)), \phi+z(s)) \rightarrow(M(\phi), \phi)>0 .
$$

Hence $\inf \sigma\left(L_{\lambda}^{1}\right) \leq\left(L_{\lambda}^{1} \varphi_{\lambda}, \varphi_{\lambda}\right) /\left(\varphi_{\lambda}, \varphi_{\lambda}\right)<0$. But there exists $\varepsilon>0$ such that $\sigma(L) \cap(-\infty, \Lambda+\varepsilon)=\{\Lambda\}$ and $\Lambda$ is a simple eigenvalue of $L$. Since $L_{\lambda}^{1} \rightarrow L-\Lambda$ in $B\left(H^{2}\left(\mathbb{R}^{N}\right), L^{2}\left(\mathbb{R}^{N}\right)\right)$ as $\lambda \rightarrow \Lambda$, this implies that for $\lambda$ near $\Lambda,\left(-\infty, \frac{\varepsilon}{2}\right)$ contains exactly one eigenvalue of $L_{\lambda}^{1}$ and it is simple. Thus $M\left(L_{\lambda}^{1}\right)=1$ and $\operatorname{ker} L_{\lambda}^{1}=\{0\}$.

Thus we see that the properties required in condition (5) of the summary at the end of Section 7 is satisfied and hence orbital stability now follows from Proposition 5.2.

(II) Case $V \leq 0$ and $V<0$ on a set of positive measure.

For all $v \in H^{2}(\mathbb{R}) \backslash\{0\}$, we have that

$$
\left(L_{\lambda}^{1} v, v\right)=\left(L_{\lambda}^{2} v, v\right)-(p-1) \int_{\mathbb{R}^{N}} V|v|^{p+1} d x \geq\left(L_{\lambda}^{2} v, v\right) \geq \inf \sigma\left(L_{\lambda}^{2}\right)=0
$$

and so $\inf \sigma\left(L_{\lambda}^{1}\right) \geq 0$. If $\inf \sigma\left(L_{\lambda}^{1}\right)=0,0$ must be an eigenvalue of $L_{\lambda}^{1}$ with a positive eigenfunction $\psi>0$. (See Theorem 3.20 in [29], for example.) But then $0=L_{\lambda}^{1} \psi=L_{\lambda}^{2} \psi-(p-1) V \psi^{p-1}$ and so $0=\left(L_{\lambda}^{1} \psi, \varphi_{\lambda}\right)=\left(L_{\lambda}^{2} \psi, \varphi_{\lambda}\right)-(p-1)\left(V \psi^{p-1}, \varphi_{\lambda}\right)=-(p-1)\left(V \psi^{p-1}, \varphi_{\lambda}\right)$ since $L_{\lambda}^{2} \varphi_{\lambda}=0$. From the properties of $\varphi_{\lambda}, \psi$ and $V$ we have that $\left(V \psi^{p-1}, \varphi_{\lambda}\right)<0$, contradicting the preceding conclusion. Hence inf $\sigma\left(L_{\lambda}^{1}\right)>$ 0 .

Recalling (8.1), this establishes the property (4) in the summary at the end of Section 7. Orbital stability now follows from Corollary 3.4. 
Remark. We observe that in the defocusing case (II), we have shown that the Hessian $D_{v v}^{2} G\left(\xi_{\lambda}\right)$ of the augmented Hamiltonian is actually positive definite on

$$
\left\{\left(\begin{array}{c}
v \\
z
\end{array}\right) \in H^{1}\left(\mathbb{R}^{N}\right) \times H^{1}\left(\mathbb{R}^{N}\right): \int_{\mathbb{R}^{N}} z \varphi_{\lambda} d x=0\right\} \text { where } \xi_{\lambda}=\left(\begin{array}{c}
\varphi_{\lambda} \\
0
\end{array}\right) .
$$

\subsection{Global branch in the defocusing case}

Theorem 8.1 deals with bifurcation from a simple eigenvalue and yields a local branch of standing waves with frequencies near $\Lambda$. In the defocusing case, the branch has been extended globally under the following assumptions.

In addition to the assumptions $(\mathrm{C} 1)$ and $(\mathrm{C} 2)$ we suppose that

$$
P, V \in C^{1}\left(\mathbb{R}^{N}\right) \text { with } V(x)<0 \text { for all } x \in \mathbb{R}^{N} \text { and } \lim _{|x| \rightarrow \infty} P(x)=0 .
$$

Furthermore, we suppose that there exists $C>0$ such that

$$
P(x) \leq C|V(x)| \text { for all } x \in \mathbb{R}^{N} \text { and } \lim \sup _{|x| \rightarrow \infty}|x|^{2} P(x) \leq 0 .
$$

If $N \leq 2$, we suppose also that there exist $b \in[0,2)$ and $L \in(0, \infty)$ such that $\lim _{|x| \rightarrow \infty}|x|^{b}|V(x)|=L$.

It follows from the assumption $\lim _{|x| \rightarrow \infty} P(x)=0$ that $0=\inf \sigma(-\Delta-$ $P$ ) and hence from (C2) that $\Lambda<0$.

The existence of a global branch of real stationary solution was established in [10] and it turns out that the corresponding standing waves are all orbitally stable.

Theorem 8.2. Let the conditions $(\mathrm{C} 1),(\mathrm{C} 2)$ and $(\mathrm{C} 3)$ be satisfied. There exists a function $\varphi \in C\left((\Lambda, 0), H^{2}\left(\mathbb{R}^{N}\right)\right)$ having the following properties

$$
\begin{aligned}
& \varphi(\lambda)=\varphi_{\lambda} \in C^{1}\left(\mathbb{R}^{N}\right) \cap L^{\infty}\left(\mathbb{R}^{N}\right), \quad \varphi_{\lambda}>0 \text { on } \mathbb{R}^{N}, \\
& \text { there exists } \mu(\lambda)>0 \text { such that } \lim _{|x| \rightarrow \infty} e^{\mu(\lambda)|x|} \varphi_{\lambda}(x)=0, \\
& \lim _{\lambda \rightarrow \Lambda}\left\|\varphi_{\lambda}\right\|_{H^{2}\left(\mathbb{R}^{N}\right)}=\lim _{\lambda \rightarrow \Lambda}\left\|\varphi_{\lambda}\right\|_{L^{\infty}\left(\mathbb{R}^{N}\right)}=0, \\
& \varphi_{\mu}(x)>\varphi_{\lambda}(x)>0 \text { for all } x \in \mathbb{R}^{N} \text { and } \Lambda<\lambda<\mu<0, \\
& \Delta \varphi_{\lambda}+P \varphi_{\lambda}+V \varphi_{\lambda}^{p}+\lambda \varphi_{\lambda}=0 \text { on } \mathbb{R}^{N} .
\end{aligned}
$$

Furthermore, for each $\lambda \in(\Lambda, 0), \varphi_{\lambda}$ is the unique positive solution of (8.5) and so $\left(\lambda, \varphi_{\lambda}\right)$ is an extension of the branch given by case (II) of Theorem 8.1. If $\lambda \notin[\Lambda, 0]$, then (8.5) has no positive solution. 
For all $\lambda \in(\Lambda, 0)$ the standing wave generated by $\varphi_{\lambda}$ is orbitally stable.

The existence and properties of the global branch parametrized by $(\Lambda, 0)$ are proved in Theorem 10 of [10] for a broader class of nonlinearities. In [10] there is also a detailed study of the behaviour of the branch as $\lambda \rightarrow 0$.

Concerning the stability of the corresponding standing waves, we note that, by Corollary 6.1.2 of [4], assumptions (C1) and (C3) ensure that (GWP at $\xi$ ) holds for all $\xi \in H^{1}\left(\mathbb{R}^{N}\right) \times H^{1}\left(\mathbb{R}^{N}\right)$. For all $\lambda \in(\Lambda, 0)$, the discussion of the operators $L_{\lambda}^{1}$ and $L_{\lambda}^{2}$ for any $\lambda \in(\Lambda, 0)$ is the same as for Case (II) of Theorem 8.1. Thus the condition $\left(\mathrm{SC}^{* *}\right)$ is satisfied and orbital stability follows from Corollary 3.4.

\subsection{Global branch in the self-focusing case}

In the self-focusing case, a global branch of stable standing waves has only been established when $N=1$ and under much more restrictive hypotheses on $P$ and $V$.

(J) $N=1, p>1$ and $P, V \in C^{1}(\mathbb{R}, \mathbb{R})$ satisfy

$$
\begin{aligned}
& P(x)=P(-x), P^{\prime}(x) \leq 0 \text { for all } x \geq 0 \text { and } P(0)>\lim _{x \rightarrow \infty} P(x)=0 \\
& V(x)=V(-x), V^{\prime}(x) \leq 0 \text { for all } x \geq 0 \text { and } \lim _{x \rightarrow \infty} V(x)>0 .
\end{aligned}
$$

In this context the operator $L u=-u^{\prime \prime}-P u$ introduced earlier has the properties that

$$
\Lambda=\inf \sigma(L)<0=\inf \sigma_{e}(L)
$$

and the eigenfunction $\phi$ is such that

$$
\phi(x)=\phi(-x)>0 \text { and } \phi^{\prime}(x)<0 \text { for all } x>0.1
$$

In particular, $(\mathrm{J})$ implies $(\mathrm{C} 1)$ and $(\mathrm{C} 2)$.

Theorem 8.3. Under the assumption $(\mathrm{J})$ there is a function $\varphi \in C^{1}\left((-\infty, \Lambda), H^{2}(\mathbb{R})\right)$ having the following properties.

$$
\begin{aligned}
& \lim _{\lambda \rightarrow \Lambda}\left\|\varphi_{\lambda}\right\|_{H^{2}(\mathbb{R})}=0 \text { and } \lim _{\lambda \rightarrow-\infty}\left\|\varphi_{\lambda}\right\|_{H^{2}(\mathbb{R})}=\infty . \\
& \varphi_{\lambda}(x)=\varphi_{\lambda}(-x)>0, \frac{d}{d x} \varphi_{\lambda}(x)<0 \text { for all } x>0, \\
& \text { there exists } \mu(\lambda)>0 \text { such that } \lim _{|x| \rightarrow \infty} e^{\mu(\lambda)|x|} \varphi_{\lambda}(x)=0, \\
& \varphi_{\lambda}^{\prime \prime}+P \varphi_{\lambda}+V \varphi_{\lambda}^{p}+\lambda \varphi_{\lambda}=0 \text { for all } x \in \mathbb{R} .
\end{aligned}
$$


Furthermore, all positive solutions $(\lambda, u) \in \mathbb{R} \times H^{2}(\mathbb{R})$ of (8.7) lie on this curve. Thus the solutions $\left(\lambda, \varphi_{\lambda}\right)$ form a global extension of the branch found in Theorem 8.1(I).

These conclusions are proved for a class of equations including (8.7) in [19].

Unlike the defocusing situation, it is not the case that all standing waves generated by this global branch are orbitally. For large frequencies $|\lambda|$ they may be unstable when $p$ is large. However, for $p<5$ we do have orbital stability on the whole branch.

Theorem 8.4. Suppose that $(\mathrm{J})$ is satisfied and that $1<p<5$. Consider the branch $\varphi \in C^{1}\left((-\infty, \Lambda), H^{2}(\mathbb{R})\right)$ given by Theorem 8.3. For all $\lambda \in$ $(-\infty, \Lambda)$, the standing wave generated by $\varphi_{\lambda}$ is orbitally stable.

The stability is established by using the criterion (5) in the summary at the end of Section 7. In [24] it is proved that, if $1<p \leq 5$,

$$
\frac{d}{d \lambda}\left\|\varphi_{\lambda}\right\|_{L^{2}}^{2}<0 \text { for all } \lambda<\Lambda
$$

and so the condition (VK) is satisfied. In the present context

$$
\begin{aligned}
& L_{\lambda}^{1} z=L z-P z-p V \varphi_{\lambda}^{p-1} z-\lambda z \text { with } D\left(L_{\lambda}^{1}\right)=H^{2}(\mathbb{R}) \text { and } \\
& L_{\lambda}^{2} z=L z-P z-V \varphi_{\lambda}^{p-1} z-\lambda z \text { with } D\left(L_{\lambda}^{1}\right)=H^{2}(\mathbb{R}) .
\end{aligned}
$$

Standard results show that all eigenvalues of $L_{\lambda}^{1}$ and $L_{\lambda}^{2}$ are simple, $L_{\lambda}^{1} \leq L_{\lambda}^{2}$ and

$$
\inf \sigma_{e}\left(L_{\lambda}^{1}\right)=\inf \sigma_{e}\left(L_{\lambda}^{2}\right)=-\lambda>-\Lambda>0 \text { for all } \lambda<\Lambda,
$$

since $\lim _{|x| \rightarrow \infty} P(x)=\lim _{|x| \rightarrow \infty} \varphi_{\lambda}^{p-1}(x)=0$. Furthermore, $0=\inf \sigma\left(L_{\lambda}^{2}\right)$ by the positivity of $\varphi_{\lambda}$, as before. It is proved in Lemma 3.4 of ([30]) that, for all $\lambda<\Lambda$,

$$
0 \notin \sigma\left(L_{\lambda}^{1}\right) \text { and } L_{\lambda}^{1} \text { has exactly one negative eigenvalue. }
$$

Thus the spectral conditions required in criterion (5) of the summary in Section 7 are satisfied. By Corollary 6.1.2 of [4], the condition (GWP at $\xi)$ holds for all $\xi \in H^{1}(\mathbb{R})$ when $1<p<5$. Now we can conclude from Proposition 5.2 that the associated standing waves are all orbitally stable. 


\subsection{The case where $P$ is constant}

As pointed out before, $(\mathrm{C} 2)$ cannot hold when $P$ is constant. In that case, by a change of variable, we can suppose that $P \equiv 0$ and so

$$
f\left(x,|w|^{2}\right)=V(x)|w|^{p-1},
$$

where we now assume

(K) $N \geq 3, V \in C^{1}\left(\mathbb{R}^{N} \backslash\{0\}\right)$ and there exists $b \in(0,2)$ such that

$$
\begin{aligned}
& \lim _{|x| \rightarrow \infty}|x|^{b} V(x)=1 \text { and } \lim \sup _{|x| \rightarrow 0}|x|^{b}|V(x)|<\infty . \text { Also } \\
& \lim _{|x| \rightarrow \infty}|x|^{b} W(x)=0 \text { and } \lim \sup _{|x| \rightarrow 0}|x|^{b}|W(x)|<\infty \\
& \text { where } W(x)=x \cdot \nabla V(x)+b V(x) .
\end{aligned}
$$

This implies that $V>0$ for $|x|$ large but $V$ may change sign. Note also that we are no longer requiring that $V \in L^{\infty}\left(\mathbb{R}^{N}\right)$.

It is known (see [13], for example) that, for $b \in(0,2)$ and $1<p<$ $1+\frac{4-2 b}{N-2}$, there is a unique positive function $\psi \in H^{1}\left(\mathbb{R}^{N}\right) \cap C\left(\mathbb{R}^{N}\right)$ satisfying

$$
\Delta \psi-\psi+|x|^{-b} \psi^{p}=0 .
$$

This function is radially symmetric and radially non-increasing.

Theorem 8.5. Let the $V$ satisfy the condition $(\mathrm{K})$.

(i) If $1<p<1+\frac{4-2 b}{N-2}$, there exist $\delta>0$ and a function $\varphi \in C^{1}\left((-\delta, 0), H^{1}\left(\mathbb{R}^{N}\right)\right)$ having the following properties.

$\varphi_{\lambda}=\varphi(\lambda) \in C\left(\mathbb{R}^{N}\right) \cap L^{\infty}\left(\mathbb{R}^{N}\right)$ and $\varphi_{\lambda}(x)>0$ for $x \neq 0$,

there exists $\mu(\lambda)>0$ such that $\lim _{|x| \rightarrow \infty} e^{\mu(\lambda)|x|} \varphi_{\lambda}(x)=0$,

$\varphi_{\lambda}$ is a weak solution of $\Delta \varphi_{\lambda}+V \varphi_{\lambda}^{p}+\lambda \varphi_{\lambda}=0$ on $\mathbb{R}^{N}$,

$\varphi_{\lambda}(x)=|\lambda|^{\frac{2-b}{2(p-1)}}\left\{\psi\left(|\lambda|^{\frac{1}{2}} x\right)+v_{\lambda}(x)\right\}$ where $\left\|v_{\lambda}\right\|_{H^{1}} \rightarrow 0$ as $\lambda \rightarrow 0$.

(ii) If $1<p<1+\frac{4-2 b}{N}$, then the standing wave generated by $\varphi_{\lambda}$ is orbitally stable.

In this case, for $1<p<1+\frac{4-2 b}{N}$, the solutions $\varphi_{\lambda}$ bifurcate from the bottom of the essential spectrum and not from an eigenvalue of the linearization as in Theorem 8.1. After an appropriate rescaling of the variables, this result is proved in [13] using Proposition 5.2. Earlier related results were proved using the condition $\left(\mathrm{SC}^{*}\right),[9],[20]$. 


\subsection{Conditions ensuring weak stability}

The weaker stability property (WS) that is established in Theorem 6.2 is easier to prove and hence can be obtained under weaker hypotheses on the coefficients $P$ and $V$, at least for $1<p<1+\frac{4}{N}$. Note however that Theorem 8.1 allows $1<p<2^{*}-1\left(=1+\frac{4}{N-2}\right.$ when $\left.N \geq 3\right)$. On the other hand, the hypotheses of Theorem 8.6 below do not imply that $(\mathrm{C} 2)$ holds and so Theorem 8.1 may not be applicable under the assumptions of this section.

Recalling that for (CNLS),

$$
\begin{aligned}
X & =H^{1}\left(\mathbb{R}^{N}\right) \times H^{1}\left(\mathbb{R}^{N}\right), \\
H\left(\left(\begin{array}{c}
\varphi \\
\psi
\end{array}\right)\right) & =\frac{1}{2} \int_{\mathbb{R}^{N}}|\nabla \varphi|^{2}+|\nabla \psi|^{2}-F\left(x, \varphi^{2}+\psi^{2}\right) d x, \\
Q\left(\left(\begin{array}{c}
\varphi \\
\psi
\end{array}\right)\right) & =\frac{1}{2} \int_{\mathbb{R}^{N}} \varphi^{2}+\psi^{2} d x
\end{aligned}
$$

we see that in this context, for $c>0$,

$$
\begin{aligned}
& S(c)=\left\{(\varphi, \psi) \in X: \int_{\mathbb{R}^{N}} \varphi^{2}+\psi^{2} d x=2 c\right\} \text { and } \\
& M(c)=\{(\varphi, \psi) \in S(c): H(\varphi, \psi)=m(c)\} \\
& \text { where } m(c)=\inf \{H(\varphi, \psi):(\varphi, \psi) \in S(c)\} .
\end{aligned}
$$

Returning to the complex notation $w=\varphi+i \psi$, we we can write

$$
\begin{aligned}
& S(c)=\left\{w \in H^{1}\left(\mathbb{R}^{N}, \mathbb{C}: \int_{\mathbb{R}^{N}}|w|^{2} d x=2 c\right\}\right. \text { and } \\
& M(c)=\{w \in S(c): H(w)=m(c)\} \\
& \text { where } H(w)=\frac{1}{2} \int_{\mathbb{R}^{N}}|\nabla w|^{2}-F\left(x,|w|^{2}\right) d x \\
& \text { and } m(c)=\inf \{H(w): w \in S(c)\} .
\end{aligned}
$$

Theorem 8.6. Consider $1<p<1+\frac{4}{N}$ and $P, V \in L^{\infty}\left(\mathbb{R}^{N}\right)$ with $V \geq 0$ on $\mathbb{R}^{N}$ and $V>0$ on a set of positive measure.

(I) (compact case) If $\lim _{|x| \rightarrow \infty} V(x)=0$, there exists $c_{0} \geq 0$ such that, for all $c>c_{0}, M(c) \neq \emptyset$ and, for any $v_{c} \in M(c)$, the standing wave generated by $v_{c}$ has the weak stability property (WS).

In general, $c_{0}>0$, but $c_{0}=0$ provided that

(i) $\sup \left\{|x|^{2}\left[P(x)-\lim \sup _{|x| \rightarrow \infty} P(x)\right]_{-}: x \in \mathbb{R}^{N}\right\}<\infty$, where $[a]_{-}=\max \{0,-a\}$, 
(ii) there exist $z \in \mathbb{R}^{N}$ and constants $B>0, b \in(0,2)$ such that

$$
\begin{aligned}
1 & <p<1+\frac{4-2 b}{N} \text { and } \\
V(x) & \geq B|x|^{-b} \text { for all } x \in \Omega=\{s y: s \geq 1 \text { and }|y-z| \leq 1\} .
\end{aligned}
$$

(II) (non-compact cases) Suppose that there exist $P^{\infty}, V^{\infty} \in L^{\infty}\left(\mathbb{R}^{N}\right)$ having the following properties

$P^{\infty}(x+z)=P^{\infty}(x)$ and $V^{\infty}(x+z)=V^{\infty}(x) \geq 0$ for all $x \in \mathbb{R}^{N}, z \in \mathbb{Z}^{N}$, $P(x) \geq P^{\infty}(x)$ and $V(x) \geq V^{\infty}(x)$ for all $x \in \mathbb{R}^{N}$,

$P(x)-P^{\infty}(x) \rightarrow 0$ and $V(x)-V^{\infty}(x) \rightarrow 0$ as $|x| \rightarrow \infty$.

Then, for all $c>0, M(c) \neq \emptyset$ and, for any $v_{c} \in M(c)$, the standing wave generated by $v_{c}$ has the property (WS).

Remark. Case (II) deals with the situation where $P$ and $V$ are asymptotically periodic, possibly asymptotically constant.

Note that, when there is equality in (8.8) for all $x \in \mathbb{R}^{N}$, the coefficients $P$ and $V$ are periodic with the same period cell. For further work on this situation see [8] and [26].

Proof. Since $1<p<1+\frac{4}{N}$ and $P, V \in L^{\infty}\left(\mathbb{R}^{N}\right)$, it follows from Corollary 6.1.2 of [4] that (GWP at $\xi$ ) holds for all $\xi \in X$. By Theorem 6.2, it is enough so show that $(\mathrm{M})_{c}$ is satisfied for the relevant values of $c$. In case (I) this follows from Proposition 5.1 of [18]. In case (II) we use Proposition 5.3 of [18] if at least one of the inequalities in (8.8) is strict on a set of positive measure. If this is not the case, then $P \equiv P^{\infty}$ and $V \equiv V^{\infty}$ and we are in the context of assumption (E) of Section 6 with $\Upsilon=\left\{\gamma_{z}: z \in \mathbb{Z}^{N}\right\}$ where $\gamma_{z}(v)=v(\cdot+z)$ for all $v \in X$. Proposition 5.2 of [18] shows that $(\mathrm{EM})_{c}$ and (WS) follows from Corollary 6.3.

Remark. To understand properly the content of the property (WS) one needs to investigate further the set $M(c)$. For $P \equiv 0$ and $V \equiv 1$ a complete characterization of $M(c)$ has been achieved, but even in this case, it is a difficult problem, see [4], Chapter 8. The conclusion is that

$$
M(c)=\left\{e^{i \theta} \psi_{c}(\cdot+z): \theta \in \mathbb{R} \text { and } z \in \mathbb{R}^{N}\right\}
$$

where $\left(\lambda_{c}, \psi_{c}\right) \in \mathbb{R} \times H^{1}\left(\mathbb{R}^{N}\right)$ is the unique radially symmetric positive solution of the stationary equation

$$
\Delta \psi+\psi^{p}+\lambda \psi=0 \text { on } \mathbb{R}^{N} \text { with } \int_{\mathbb{R}^{N}} \psi^{2} d x=2 c .
$$


In the more general context of Theorem 8.6, it is shown in Theorem 4.1 of [18] that

$$
M(c)=\left\{e^{i \theta} \psi: \theta \in \mathbb{R} \text { and } \psi \in W(c)\right\}
$$

where

$$
W(c)=\left\{\psi \in H^{1}\left(\mathbb{R}^{N}\right) \cap C^{1}\left(\mathbb{R}^{N}\right) \cap S(c): \psi>0 \text { and } H(\psi)=m(c) .\right.
$$

However a complete description of $W(c)$ is still lacking.

\section{References}

[1] A. Ambrosetti and G. Prodi. A Primer of Nonlinear Analysis, Cambridge University Press, Cambridge, 1993

[2] T. B. Benjamin, The stability theory of solitary waves, Proc. Royal Soc. London, 328 (1972), 153-183

[3] J. Bona, On the stability theory of solitary waves, Proc. Royal Soc. London, 344 (1975), 363-374

[4] T. Cazenave, Semilinear Schrödinger equations, Courant Lecture notes in Mathematics, AMS 2003

[5] T. Cazenave and P.-L. Lions, Orbital stability of standing waves for some nonlinear Schrödinger equations, Comm. Math. Phys., 85 (1982), 549-561

[6] A. Comech and D. Pelinovsky, Purely nonlinear instability of standing waves with minimal energy, Comm. Pure Appl. Math., LVI (2003), 1565-1607

[7] M. G. Crandall and P. H. Rabinowitz, Bifurcation from simple eigenvalues, J. Functional Anal., 8 (1971), 321-340

[8] S. Cuccagna, Stability of standing waves for NLS with perturbed Lamé potential, J. Differntial Equations, 223 (2006), 112-160

[9] A. de Bouard, and R. Fukuizumi, Stability of standing waves for nonlinear Schrödinger equations with inhomogeneous nonlinearities, Ann. Henri Poincaré 6 (2005), 1157-1177.

[10] A. Edelson and C. A. Stuart, The principal branch of solutions of a nonlinear elliptic eigenvalue problem on $\mathbb{R}^{N}$, J. Diff. Equat., 124 (1996), 279 -301

[11] M. Esteban and W. Strauss, Nonlinear bound states outside an insulated sphere, Comm. Partial Differential Equations, 19 (1994), 177-197

[12] R. Fukuizumi, Stability and instability of standing waves for nonlinear Schrödinger equations, Tohoku Mathematical Publications, No 25, Tohoku University, Japan, 2003

[13] F. Genoud and C. A. Stuart, Schrödinger equation with a spatially decaying nonlinearity, Existence and stability of standing waves, Discrete \& Continuous Dynamical Systems, 21 2008, 137-186 
[14] M. Grillakis, J. Shatah and W. Strauss, Stability theory of solitary waves in the presence of symmetry, I, J. Functional Anal., 74 (1987), 160-190

[15] M. Grillakis, J. Shatah and W. Strauss, Stability theory of solitary waves in the presence of symmetry, II, J. Functional Anal., 90 1990, 308-348

[16] M. Grillakis, M. Linearized instability for nonlinear Schrödinger and KLeinGordon equations, Comm. Pure Appl. Math., XLI (1988), 747-774

[17] M. Grillakis, Analysis of the linearization around a critical point of an infinite dimensional dynamical system, Comm. Pure Appl. Math., XLIII (1990), 299333

[18] H. Hajaiej and C. A. Stuart, On the variational approach to the stability of standing waves for the nonlinear Schrödinger equation, Adv. Nonlinear Studies, 4 (2004), 469-501

[19] H. Jeanjean and C. A. Stuart, Nonlinear eigenvalue problems having an unbounded branch of symmetric bound states, Adv. Differential Equations, 4 (1999), $639-670$

[20] L. Jeanjean and S. Le Coz, An existence and stability result for standing waves of nonlinear Schrödinger equations, Adv. Differential Equations, 11 (2006), 813-840.

[21] T. Kato, Perturbation Theory for Linear Operators, Springer, Berlin 1967

[22] Y. S. Kivshar and A. A. Sukkhorukov, Stability of spatial optical solitons, in Spatial Optical Solitons, editors T. Torruellas and S. Trillo, Springer, New York 2001

[23] J. H. Maddocks and R. L. Sachs, On the stability of KdV multi-solitons, Comm. Pure Appl. Math., XLVI (1993), 867-901

[24] J. B. McLeod, C. A. Stuart and W. C. Troy, Stability of standing waves for some nonlinear Schrödinger equations, J. Differential Integral Equations, 16 (2003), 1025 - 1038

[25] T. Mizumachi, A remark on linearly unstable standing wave solutions to NLS, Nonlinear Anal. TMA, 64 (2006), 657-676

[26] D. E. Pelinovsky, A. A. Sukhorukov and Y. S. Kivshar, Bifurcations and stability of gap solitons in periodic potentials, Phys. Rev. E, 70 (2004),

[27] H. A. Rose and M. I. Weinstein, On the bound states of the nonlinear Schrödinger equation with a linear potential, Physica D, 30 (1988), 207 - 218

[28] A. Sofer and M. I. Weinstein, Selection of ground states for nonlinear Schrödinger equations, Rev. Math. Phys., 16 (2004), 977-1071

[29] C. A. Stuart, An introduction to elliptic equations on $\mathbb{R}^{N}$, in Nonlinear Functional Analysis and Applications to Differential Equations, editors A. Ambrosetti, K.-C. Chang and I. Ekeland, World Scientific, Singapore, 1998 
[30] C. A. Stuart, Uniqueness and stability of ground states for some nonlinear Schrödinger equations, J. Eur. Math. Soc., 8 (2006), 399-414

[31] C. A. Stuart, Existence and stability of TE modes in a stratified non-linear dielectric, IMA J. of Applied Math. , 72 (2007), 659-679

[32] M. G. Vakhitov and A. A. Kolokolov, Stationary solutions of the wave equation in a medium with nonlinearity saturation, Radiophys. Quantum Electron., 16 (1973), $783-785$

[33] T.-P. Tsai and H.-T. Yau, Asymptotic dynamics of nonlinear Schrödinger equations: Resonance dominated and radiation dominated solutions, Comm. Pure Appl. Math., LV 2002, 153-216

[34] M. I. Weinstein, Lyapunov stability of ground states of nonlinear dispersive evolution equations, Comm. Pure Appl. Math., XXXIX (1986), 51-68

[35] V. E. Zakharov and A. B. Shabat, Exact theory of two-dimensional selffocusing and one-dimensional self-modulation of waves in nonlinear media, Soviet Physics JETP, 34 (1972), 62 - 69

[36] P. E. Zhidkov, Korteweg de Vries and Nonlinear Schrödinger Equations: Qualitative Theory, Lecture Notes in Mathematics No 1756, Springer-Verlag, Berlin, 2001

\section{Acknowledgment}

These notes cover, in a somewhat expanded form, the contents of some lectures I gave that the Universities of Milan and Milan-Bicocca in May 2007. I wish to thank particularly, Professors Serra, Tarallo and Terracini for the invitation and for their warm hospitality. Their interest and questions stimulated me to try to improve and complete the rough notes which I had at the time. While working on these notes, I profited greatly from discussions with François Genoud and Matthieu Léautaud here at EPFL.

\section{C.A. Stuart}

Station 8

Ecole Polytechnique Fédérale Lausanne

CH-1015 Lausanne

Switzerland

e-mail: charles.stuart@epfl.ch

Received: January 2008 